\title{
PALEO
}

Revue d'archéologie préhistorique

$22 \mid 2011$

Varia

\section{Le Quercy au cœur du dernier maximum glaciaire}

La couche 4 du Petit Cloup Barrat et la question de la transition badegoulo-magdalénienne

At the heart of the Last Glacial Maximum in Quercy region: the contribution of "Le Petit Cloup Barrat » layer 4 to the Badegoulian-to-Magdalenian transition debate

Sylvain Ducasse, Jean-Christophe Castel, François-Xavier Chauvière, Mathieu Langlais, Hubert Camus, André Morala et Alain Turq

\section{(2) OpenEdition}

Édition électronique

URL : http://journals.openedition.org/paleo/2098

DOI : $10.4000 /$ paleo.2098

ISSN : 2101-0420

Éditeur

SAMRA

\section{Édition imprimée}

Date de publication : 1 décembre 2011

Pagination : 101-154

ISSN : 1145-3370

\section{Référence électronique}

Sylvain Ducasse, Jean-Christophe Castel, François-Xavier Chauvière, Mathieu Langlais, Hubert Camus, André Morala et Alain Turq, «Le Quercy au cœur du dernier maximum glaciaire », PALEO [En ligne], 22 I 2011, mis en ligne le 16 avril 2012, consulté le 07 juillet 2020. URL : http://journals.openedition.org/ paleo/2098; DOI : https://doi.org/10.4000/paleo.2098

\section{(c) $(1)$}

PALEO est mis à disposition selon les termes de la licence Creative Commons Attribution - Pas d'Utilisation Commerciale - Pas de Modification 4.0 International. 


\title{
LE QUERCY AU COEUR DU DERNIER MAXIMUM GLACIAIRE La couche 4 du Petit Cloup Barrat et la question de la transition badegoulo-magdalénienne
}

\author{
Sylvain DUCASSE(1), Jean-Christophe CASTEL ${ }^{(2)}$, \\ François-Xavier CHAUVIERE ${ }^{(3)}$, Mathieu LANGLAIS ${ }^{(4)}$, Hubert CAMUS ${ }^{(5)}$, \\ André MORALA ${ }^{(6)}$ et Alain TURQ ${ }^{(7)}$
}

\begin{abstract}
Résumé : Signalé dès les années cinquante, le gisement quercinois du Petit Cloup Barrat a été redécouvert au milieu des années quatre-vingt dix. Cette doline, située à 800 mètres de l'entrée préhistorique de la grotte de Pech Merle, a fait l'objet de fouilles programmées entre 2004 et 2008. Menée aux abords d'une grotte-abri localisée à l'extrémité ouest de la doline, cette opération a permis la mise en évidence de plusieurs niveaux d'occupations datant de la seconde moitié du Paléolithique supérieur. Deux secteurs ont été distingués, à partir des différentes signatures sédimentaires de la séquence stratigraphique : la zone avant, qui correspond à la partie « ouverte » de l'espace fouillé, et la zone du fond, située sous le porche. Dans la grotte, affectée par des phénomènes de soutirage karstique, le remplissage mis au jour documente une succession d'au moins trois techno-complexes distincts : la couche10N, dont l'attribution reste à confirmer, fait suite à l'ensemble 8 où se succèdent Solutréen supérieur, Badegoulien à raclettes et Magdalénien inférieur. Dans la zone avant, le Magdalénien moyen de la couche 3, remanié par la mise en culture de la doline, coiffe la couche 4, ensemble le mieux documenté à ce jour et qui fait l'objet de cet article.
\end{abstract}

Riche de plusieurs milliers de vestiges, cet ensemble présente certaines particularités : si la mauvaise préservation de la faune limite l'approche archéozoologique, les données acquises lors de l'analyse des productions lithiques et osseuses apportent en revanche de nombreux éléments de discussion. La composante lithique, à travers la nature de certaines armatures et de leurs modalités de production, fait clairement écho à certaines industries du Magdalénien inférieur. Néanmoins, tandis que la plus grande part de la composante osseuse s'accorde pleinement avec cette proposition (type de pointe, extraction par rainurage longitudinal), une série de pièces témoigne de la mise en œuvre d'un débitage du bois de renne par percussion habituellement exclu de ces ensembles. Cette « mixité » technique qu'il conviendra de confirmer par le biais de datations directes - vient alimenter les débats actuels sur la transition badegoulo-magdalénienne. Enfin, la découverte de plusieurs incisives d'herbivores sciées et d'une ébauche d'anneau ouvert en bois de renne renforce l'originalité de l'assemblage, ouvrant de nouveaux horizons de recherches.

Mots-clés : Dernier Maximum Glaciaire, Quercy, Magdalénien inférieur, Badegoulien, archéozoologie, industrie lithique, industrie osseuse, parure.

Key-words: Late Glacial Maximum, Quercy region, Lower Magdalenian, Badegoulian, archaeozoology, lithic industry, bone industry, ornaments.

(1) Laboratoire TRACES (UMR 5608) - Maison de la recherche - Université de Toulouse 2 - 5, allées Antonio-Machado, 31058 Toulouse cedex 9 - sylvain.ducasse@univ-t/se2.fr

(2) Département d'archéozoologie du Muséum d'Histoire naturelle de Genève - Route de Malagnou, CP 6434,1211 Genève 6, Suisse Jean-Christophe.Castel@ville-ge.ch

(3) Office et Musée d'Archéologie de Neuchâtel - Laténium, Espace Paul Vouga, 2068 Hauterive (Suisse) et Université Jean Moulin -

Lyon 3 - francois-xavier.chauviere@ne.ch

(4) Laboratoire PACEA (UMR 5199) - CNRS - Université de Bordeaux - Avenue des facultés - Bâtiment B18, F-33405 Talence -

m.langlais@pacea.u-bordeaux1.fr

(5) Hypogée, Géologue expert - Ancien Chemin des Ganges, 34190 Saint-Bauzille-de-Putois - hubert.camus@free.fr

(6) Laboratoire PACEA (UMR 5199) - Musée national de Préhistoire, 24620 Les Eyzies-de-Tayac - andre.morala@culture.gouv.fr

(7) Laboratoire PACEA (UMR 5199) - Musée national de Préhistoire, 24620 Les Eyzies-de-Tayac - alain.turq@culture.gouv.fr 


\section{Abridged english version}

At the heart of the Last Glacial Maximum in Quercy region: the contribution of "Le Petit Cloup Barrat » layer 4 to the Badegoulian-to-Magdalenian transition debate

The Petit Cloup Barrat site is situated in Cabrerets (Lot, France), $800 \mathrm{~m}$ to Pech Merle, a site famous for Palaeolithic cave art (fig. 1). It was discovered during the 1990 s and comprises a small $\left(20 \mathrm{~m}^{2}\right)$ cavity opening onto the edge of a doline (fig. 2). Excavations began in 2003, yielding a succession of sedimentary assemblages containing numerous mineral and organic remains attributable to the Upper Palaeolithic. The bottom of the stratigraphy has not yet been reached.

The preservation of the archaeological layers and their respective sedimentary envelopes inside and outside the shelter is not consistent. In front of the current entrance the layers show signs of severe leaching and detailed subdivision is not possible. Conversely, under the porch the sedimentary distinctions are well preserved, ensuring good preservation of the lithic and organic remains. So far, around a dozen square metres have been excavated, but an extension of more than $50 \mathrm{~m}^{2}$ is possible (fig. 2A, 3B).

\section{Research program}

The assemblage presented here belongs to layer 4, which extends in front of the shelter. It contains evidence of industries contemporary with the Last Glacial Maximum hitherto unknown from Quercy. Despite the provisional character of the sample (excavations are ongoing), an in-depth study of these findings seems important in the context of the debate surrounding the Badegoulian-Magdalenian transition.

This research has regional and inter-regional implications. On the regional level, work carried out between the late 1970 s and the first half of the 1990s helped to redefine the transition between the Badegoulian and Magdalenian techno-complexes. According to these studies and the ${ }^{14} \mathrm{C}$ data then available, these two distinct entities seem to have shared a contemporaneous phase (circa 17000 BP) but in a distinct geographical area (Lot/Célé valley vs. Aveyron valley). Further investigations, both taphonomical (evaluation of the homogeneity and representativeness of the series) and methodological (the pertinence of ${ }^{14} \mathrm{C}$ frameworks that group AMS and conventional dates), are required. Layer 4 of Petit Cloup Barrat represents a meticulous recent excavation with which to test most of the scenarios that have been advanced to explain this overlap in production methods and artefacts.

\section{Stratigraphy and main features of layer 4}

The sedimentary assemblages that make up this complex stratigraphy have been affected by various processes including drawdown, runoff, gelifraction and human and animal disturbance. A considerable part of the clastic sediment in the excavation sector results from erosion of the ledge and of the cave roof or nearby calcareous escarpments. From bottom to top, several units may be observed (fig. 3A): (1) sedimentary unit 10, included layer $10 \mathrm{~N}$ tentatively attributed to the early Solutrean; (2) layer 9 yielding a sparse archaeological content currently unattributed; (3) sedimentary unit 8 yielding Upper Solutrean assemblages - 8a1 -, Badegoulian - 8a2 - and layer 8c, in direct contact with layer 8a2, quite similar to layer 4; (4) sedimentary unit 6/5 and layer 7 contain sparse and unattributed remains; (5) Layer 4 containing the material that is the subject of this article; (5) layers 3 to 0 yielding heterogeneous archaeological assemblages (Middle Magdalenian mixed with ceramic fragments).

Layer 4 is a formation comprising an earthy matrix with blocks of several decimetres lying flat with modified surfaces, calcareous pebbles broken from the shelter and allogenic pebbles. It slopes gently towards the centre of the doline. In the sector which has been fully excavated, several vertical pebbly structures affect the thickness and reach as far as layer 9 , traversing layer 5 . At the time that it formed the site was open, and it has experienced progressive infilling and stabilisation of the slopes to the horizontal. Because of gelifraction and remobilisation, the boundaries between layer 4 and those that enclose it are difficult to define. The abundance or scarcity of large calcareous items gives information on changes in the physical geography of the shelter and the doline. The upper and lower boundaries of layer 4 have been determined by the distribution of artefacts, both being indicated by the decreasing frequency of remains.

A key question concerns the relationship between the sequence identified in front of the shelter, and that found in the covered section of the deposits (which includes items attributable to the Solutrean and Badegoulian). There are clear parallels between the characteristic remains of layer 4and those found in the shelter, despite divergences in the dynamics of sedimentation. The challenge is to position the layer 4 occupations with respect to the techno-complexes previously cited. Some explanations will be provided by means of a general archaeo-stratigraphic discussion, incorporating material found between layers $8 \mathrm{c}$ and $8 \mathrm{~d}$ and some new ${ }^{14} \mathrm{C}$ dating.

\section{Lithic production and artefacts}

An initial petrographical examination of the non-flint rock shows a mixture of local and allogenic material. Some of these show signs of use (cutting, intentional splitting and traces of abrasion or polishing), although it is not yet possible to say in exactly which processes they were used. 
Flint remains are abundant, and thanks to their distinctiveness play a central role in the chrono-cultural definition of the layer 4 archaeological assemblage. An initial petrographical examination reveals the presence of local and/or regional material (Flint from the Célé alluvium, Jurassic Portlandian flint, etc) associated with more distant material (Périgord, Haut-Agenais, southern Aquitaine basin and Saintonge).

The material includes 25,555 flint objects (tabl.1). In addition to 20,294 splinters there are 5,261 assorted pieces (flakes, blades and bladelets) of which around 15\% have been made into tools. A group of 283 objects shows that various kinds of blanks were used to create a variety of domestic tools (tab. 2; fig. 5 and 6). Although in some instances finished tools had been imported (fig. 7), the finds indicate on-site blade production in accordance with "semi-tournant» exploitation. Apart from the production of thicker flakes to be used as bladelet cores, the flake production has also been performed on the site to obtain thin flakes (fig. 8). Most of the microliths relate to hunting ( $n=394$, including manufacturing waste or unfinished pieces: see table 3) and show some original features (fig. 10, 14 and 17). One morphotype can be distinguished from numerous backed bladelets and intrusive scalene ones (Middle Magdalenian overlying: fig. 9): this consists of right and marginal backed bladelets and micro-bladelets ( $n=66$, fig. 10), whose asymmetrical shape, clearly predetermined during the debitage phases (fig. 11 and 12), is then accentuated/corrected by a consistent series of modifications (fig. 13). In the same way, the very varied production methods for (micro-)bladelets is also significant (tabl. 5): apart from pyramidal and prismatic cores made on flint blocs, there are numerous cores that show an "edge blank debitage" (débitage "sur tranche d'éclats » : fig. 16) or a "front blank debitage» (débitage "sur front d'éclat" : fig. 15). Some of which are now considered characteristic of the Lower Magdalenian like the "off-centre ventral front" debitage (fig. 15, n`5, 6 and 8).

\section{Bone remains}

Layer 4 has yielded 3,647 identifiable bone remains. Among the identified species there are considerably more reindeer than ibex or horse (tabl. 7, fig. 21A). It is difficult to determine the relative importance of reindeer and ibex in the faunal composition insofar as the splitting and fragmentation of the bones prevents more than a simple anatomical identification for some of the small fragments. The layer 4 assemblage seems to show selective hunting of smaller ungulates, even though the environment would have provided larger prey.

The diaphyses are the best represented in terms of identified fragments, and MNI (tabl. 8). The post-cranial axial bones are much rarer. Although these fragile bones are prone to destruction after deposition, it is possible that some axial bones were introduced into the habitat. Some 40 bones show cutmarks; almost all are on diaphyses, and 3 are disarticulation cuts. After taking the meat, the potential nutritional resources of the bones do not appear to have been exploited by the classic form of dismemberment, but rather by breaking the bones near the joints (fig. 22). This method allows access to the marrow of the long bones and separation of the joints for use as fuel. A large proportion of the conserved bone material is burnt (fig. 18 and 23).

Dental remains are relatively abundant, though few teeth are still attached to jaws. All age groups are represented, with a slight over-representation of young adults (fig. 21B). The summer, and the end of the good hunting season are attested, the other seasons do not seem to be represented.

\section{Hard skeletal production and artifacts}

Layer 4 has yielded 198 fragments of worked and/or used reindeer antler, bones, teeth and shells (fig. 24; tabl. 10-14).

Reindeer antler was used in the production of projectile points (fig. 26, $n^{\circ} 1-5$ ) and also objects of unknown function (pseudo baguettes demi-rondes) (fig. $26, \mathrm{n}^{\circ} 6-13$ ). Analysis has revealed the coexistence of two segmentation methods: cutting grooves to split the antler and produce rods which are then shaped by scraping and fine abrasion (fig. 25); and percussion to reduce the fragments of beam or tine before shaping them (fig. 27). All stages of the first method are present on site, but there are no examples of finished products for the second method. The discovery of an uncompleted open ring adds to the interest of this series as it gives the first certain stratigraphic placement for objects of this type, previously attested by examples from earlier excavations at Placard (Charente) and the Cave à Endives (Lot; fig. 28, graph. 4). This discovery adds to our knowledge of the diachronic links between Charente and Quercy, already suggested by symbols of the Placard type identified in the decoration of the caves of Pech Merle and Cougnac.

Two functional groups can be distinguished among the bone tools: grinders made from ungulate diaphyses and eyed needles (fig. 29).

The ornaments are remarkable for the marked presence of herbivore incisors with cuts on the vestibular surface ( $n=23)$, of which one finds the roots left over from their manufacture suggesting on-site production, as much as for the scarcity of pierced teeth $(n=2$; fig. 30A). The other animal component of the body ornamentation consists of shells $(n=3)$, principally Dentalium sp (fig. 30B).

\section{Discussion and perspectives}

The functional status of the layer 4 occupations is difficult to define, quite apart from chrono-cultural considerations. Even bearing in mind the probability that this assemblage corresponds to several distinct phases of occupation, the coexistence of hunting weapons, domestic tools and ornaments (some produced on-site) does not really seem compatible with a 
specialised hunting shelter complementing other habitations in the valley. Here, as elsewhere in Quercy during the LGM, reindeer is the main prey species during the hunting season.

Although some of the ornaments (cut herbivore incisors) found in layer 4 have no equivalent within the industries currently attributed to the Lower Magdalenian, the lithic and bone artefacts discussed above certainly belong to this techno-complex. Based on the objectives and methods of lithic production (e.g.: right and marginal backed micro-bladelets) and the methods used to work reindeer antler and the associated tools (splitting by double grooves as the most frequent method; round or elliptical points, pseudo-baguettes demi-ronds), the whole assemblage fits comfortably into the second half of the LGM. This chronological position was recently confirmed by two ${ }^{14} \mathrm{C}$ results that place the layer 4 occupations between $16,950+/-90$ $\mathrm{BP}$ and 16,370 +/- $90 \mathrm{BP}$, thereby complementing the radiometric data already available for the sequence inside the shelter. A similar lithic industry was found at the top of layer 8c, dated around 16,000 BP (15,870 +/- 150 BP), above a Badegoulian assemblage with raclettes dating to 18,598 +/- 150 BP (fig. 33).

The first evidence of the percussive method of working reindeer antler, characteristic of the Badegoulian must be examined taphonomically before integration into wider chronological considerations. It is, however, already possible to situate the industries of layer 4 of Petit Cloup Barrat in a broad network of social interactions apparent in the LGM (fig. 36). The transfer of flint raw material, certain stone and bone components, « open rings » or sandstone lamps (fig. 37) suggest that this network extends at least from the Pyrenees to Vienne, encompassing the site of Lascaux.

\section{1 - Introduction}

Mentionné par l'Abbé Lemozi dès les années cinquante (Lemozi 1951), le site du Petit Cloup Barrat fut redécouvert au milieu des années 1990 par le petit-fils d'André David, inventeur de la grotte ornée de Pech-Merle dont l'entrée préhistorique est distante d'à peine $800 \mathrm{~m}$. C'est en effet au sud du causse de Gramat, dans la commune de Cabrerets, que se localise le gisement, à une altitude de $320 \mathrm{~m}$ (fig. 1). Le paysage environnant est celui des plateaux jurassiques faiblement vallonnés et entaillés par le Lot et le Célé, cours d'eaux dont la confluence se situe à quelques kilomètres du site. L'abondance de silex taillés associés à des ossements de renne et de bouquetin sur une majeure partie de cette petite doline ${ }^{8}(35 \mathrm{~m}$ de longueur, $14 \mathrm{~m}$ de largueur et environ $2 \mathrm{~m}$ de profondeur) laissait supposer l'existence d'un vaste site dont les occupations, d'après les observations réalisées par Jacques Jaubert et Jean-Michel Geneste au début des années 2000, pouvaient être attribuées à une phase moyenne du Magdalénien.

À l'aplomb de l'entrée d'une grotte située à l'extrémité ouest de la doline se trouvait, avant le début des opérations, un « monticule » allongé $(5 \times 1 \mathrm{~m}$ sur $0,20 \mathrm{~m}$ de hauteur) correspondant probablement aux déblais des fouilles anciennes (fig. 2B1). Le produit de ces premiers travaux, dont il est aujourd'hui difficile de reconstituer la chronologie exacte, est peut-être en partie conservé au Musée de Préhistoire de Cabrerets. L'indication «Cloup Barrat » est en effet associée à une série lithique et osseuse très réduite, peu diagnostique, mais dont l'aspect général semble tout à fait compatible avec les vestiges exhumés lors des fouilles actuelles. Puisque, a priori, il ne s'agit pas des frères Brondel, amis d'André David qui ont pourtant exploité le gisement dans les années cinquante, c'est vers l'Abbé Lemozi ou André David lui-même qu'il faut se tourner pour trouver l'inventeur de cette série. Ce dernier, amateur éclairé dont nous savons qu'il a localement pratiqué de nombreuses opérations entre 1920 et 1970, a d'ailleurs conservé dans sa cave une série remarquable, redécouverte en 1994 par Pascal Gaudebert et l'un d'entre nous (JCC) : une révision récente de ce matériel lithique et osseux, publié en 1997 sous le nom de la Cave à Endives (Raux et Piel-Desruisseaux 1997), nous inciterait à y voir un autre indice de son intervention au Petit Cloup Barrat, tant les parallèles sont saisissants (cf. infra et étude en cours).

\section{1 - Une archéo-séquence classique?}

Les fortes potentialités de ce gisement ont conduit l'un d'entre nous (JCC) à y réaliser un sondage au cours de l'été 2003 (correspondant aux carrés QRSTU-71 : fig. 2A). Ce sondage, en confirmant la présence d'un ensemble Magdalénien moyen à lamelles scalènes, permit la mise en évidence de niveaux attribuables au Badegoulien à raclettes (indices remaniés) et au Solutréen récent à pointes à cran, présents notamment au Cuzoul de Vers (Vers, Lot) et aux Peyrugues (Orniac, Lot). S'ensuivit une fouille programmée menée entre 2004 et 2008 au terme de laquelle notre connaissance du site s'est affinée, tant sur le

(8) En langue occitane « cloup » signifie doline. En l'absence de toponyme précis, le nom de Petit Cloup Barrat a été donné en 2001 pour le différencier d'un cloup de plus grandes dimensions situé contre la grange cadastrée "Cloup Barrat ». Il s'est avéré plus tard que le véritable Cloup Barrat était bien celui reconnu comme site archéologique puisque le seul à être « barré ", c'est-à-dire fermé. Les deux appellations Petit Cloup Barrat et Cloup Barrat sont donc équivalentes et seront ici utilisées indifféremment. 

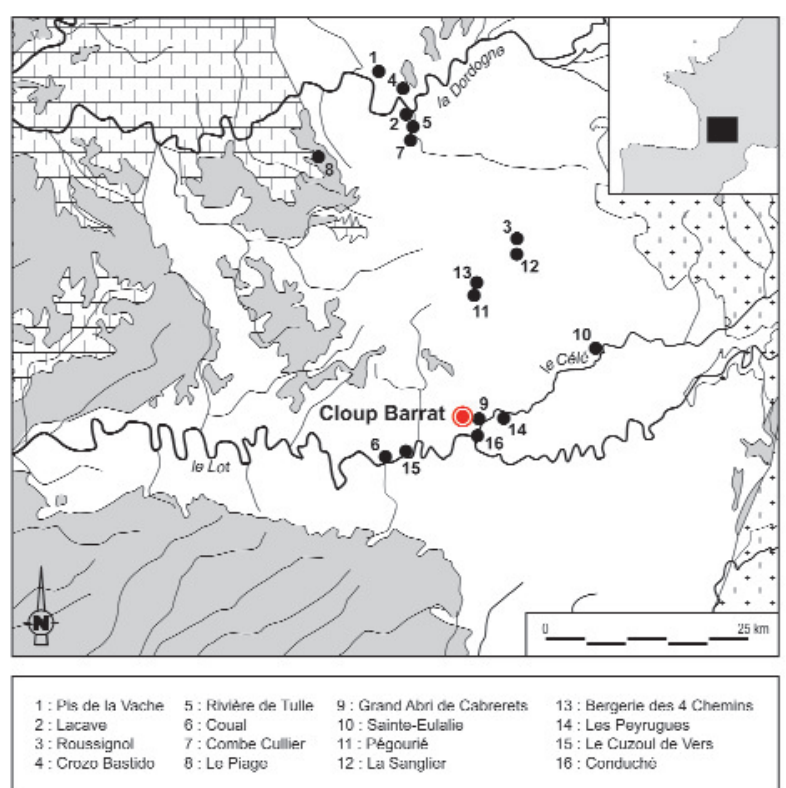

Figure 1 - Localisation du Petit Cloup Barrat et répartition des principaux gisements quercinois attribués au Solutréen, au Badegoulien et au Magdalénien.

Figure 1- Localization of the Petit Cloup Barrat and repartition of main archaeological sites in Quercy region attributed to Solutrean, Badegoulian and Magdalenian.

plan géo-archéologique que du point de vue de la caractérisation chrono-culturelle et économique des différents ensembles mis au jour. Si les travaux menés au fond de l'abri ont permis de préciser la séquence stratigraphique conservée au Petit Cloup Barrat (carrés TU-71 et 72), les trois dernières campagnes de fouille ont essentiellement concerné la couche 4 qui se développe quant à elle en dehors de l'abri. Une fouille minutieuse et adaptée aux contraintes conjuguées du terrain et de la nature des séries - nombreux micro-vestiges lithiques - a conduit à documenter un ensemble archéologique original manifestement contemporain du Dernier Maximum Glaciaire (DMG) et absent des séquences citées précédemment. L'interruption volontaire de la fouille après la campagne 2008 a été l'occasion de revenir plus précisément sur cet abondant matériel et ce, au regard des données aujourd'hui disponibles sur cet intervalle chronologique (Morala 1993 ; Fourloubey 1996 ; Cretin 2000 ; Bodu et al. dir. 2007 ; Langlais 2007 ; Ducasse 2010). Ainsi, bien que la fouille ne soit pas encore achevée, il nous a semblé important d'y consacrer une étude spécifique, cet ensemble n'ayant été le plus souvent évoqué que de manière très générale (Castel et al. 2006 ; Ducasse et Langlais 2007 ; Chauvière et al. 2008 ; Langlais et Ducasse sous presse). Son rôle dans les discussions actuellement menées sur la transition badegoulo- magdalénienne est pourtant essentiel, au même titre que certaines industries régionales (Allard 1992 ; SéronieVivien 1995 ; Clottes et al. dir. à paraître) ou non (Bodu et al. 2007 ; Langlais et al. 2007 ; Pétillon et al. 2008 ; Sacchi et al. dir. à paraître).

\section{2 - Du Quercy à l'Europe de l'ouest : rappel des enjeux}

La reprise des fouilles au Petit Cloup Barrat avait pour objectif de documenter le contraste observé dans le peuplement paléolithique entre causses et vallées quercinois. Ces deux types d'environnement étaient en effet susceptibles d'influer sur les comportements humains (conditions climatiques, spectres fauniques et végétation distincts : Castel et al. 2006). Indépendamment de problématiques plus strictement chrono-culturelles, il s'agissait de tester dans la diachronie l'hypothèse d'une complémentarité économique entre établissements de vallée (Le Cuzoul de Vers, les Peyrugues) et gisements de plateaux ; le volet archéozoologique de ce programme étant par ailleurs appuyé par les données acquises à l'Igue du Gral, piège naturel sub-contemporain permettant de préciser une grande part des ressources animales disponibles (Castel et al. 2008 ; Coumont et Castel 2007).

La fouille de la couche 4 est venue enrichir les objectifs de cette opération. Au moment où diverses analyses visaient à éclaircir la nature des relations existant entre Badegoulien et Magdalénien en Quercy, le Petit Cloup Barrat s'est posé comme un relai essentiel de cette problématique, majoritairement nourrie par des travaux réalisés entre le milieu des années 70 et le début des années 90 (Séronie-Vivien et al. 1981 ; Allard et al. 1995 ; SéronieVivien 1995). Ce renouveau de la documentation régionale, succédant à la caractérisation d'un Magdalénien inférieur aux marges méridionales du Bas-Quercy (Ladier 2000 ; Langlais 2007 ; Langlais et al. 2007), contribue depuis quelques années à cette réflexion diachronique. En effet, régionalement, deux " pôles » culturels semblent s'opposer au sein d'un même intervalle temporel. Autour de $17000 \mathrm{BP}$, tandis que le complexe Lot/Célé abrite des assemblages à raclettes attribués au Badegoulien, la vallée de l'Aveyron, dépourvue de tels indices ${ }^{9}$, se caractérise par l'existence d'industries sans raclettes intégrées au courant Magdalénien. Pour ces dernières, les arguments développés concernent tant la sphère lithique (Langlais 2007) que le travail des matières dures d'origine animale, puisque ce registre d'activité témoigne de l'emploi de la technique du double rainurage (Langlais et al. 2010b ; Pétillon com. pers.), technique généralement absente du « bagage » badegoulien (Allain et al. 1974 ; Pétillon et Averbouh à paraître ; Pétillon et Ducasse en préparation). Néanmoins, l'existence de cette manière de débiter le bois de renne à Pégourié et ce, au sein d'une industrie que les auteurs at-

(9) Indiquée par E. Ladier comme possiblement badegoulienne, la série lithique de Pénovaire (Pajot 1969) a fait l'objet d'une rapide réévaluation en Janvier 2006 par deux d'entre nous (ML et SD). Ce travail a finalement permis d'écarter cette éventualité, privilégiant son rattachement à une phase classique du Magdalénien, comme l'avait suggéré $B$. Pajot (supports laminaires dominants liés à des matériaux allochtones d'origine très variée, lamelles à dos rectilignes d'assez grand gabarit, absence totale de raclettes ou de production autonome d'éclats). 

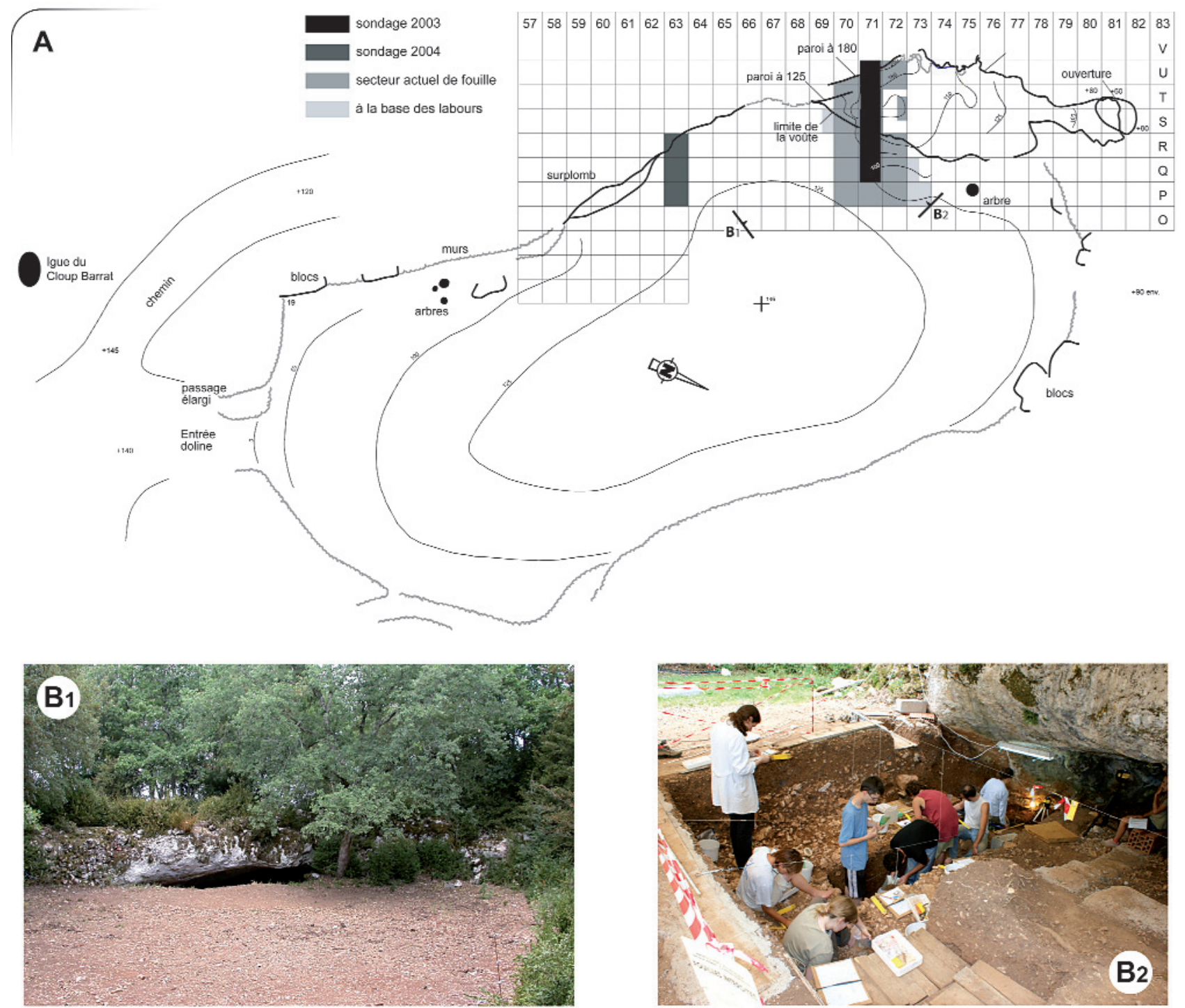

Figure 2 - A - Plan de la doline et localisation du secteur fouillé. B - Vues du site avant (1) et pendant (2) la fouille. Clichés J.-C. Castel.

Figure 2 - A - Plan of the doline and localization of the excavations. B - Views of the site before (2) and during (2) the excavations. Pictures J.-C. Castel.

tribuent, sur la base d'une forte composante lamellaire et de plusieurs datations conventionnelles, à un Badegoulien « final » à raclettes (couche 8 : Séronie-Vivien 1995, p. 254 et Séronie-Vivien 2005), alimente les débats actuels sur les termes de la transition badegoulo-magdalénienne. L'ensemble de ces éléments participe ainsi à une discussion complexe où se répondent divers arguments d'ordre synchronique (complémentarité économique, saisonnalité), diachronique (transformation des équipements), taphonomique sensu lato (évaluation de l'homogénéité et de la représentativité des séries analysées), voire méthodologique (pertinence du cadre $\mathrm{C} 14)^{10}$. En somme, la question posée est la suivante : quelles sont régionalement les réalités de cette bipartition culturelle et géographique?
In fine, à l'échelle quercinoise, ces deux principaux axes de recherche se sont donc rejoints, créant une synergie entre approche chrono-culturelle (Badegoulien final versus Magdalénien inférieur) et réflexions d'ordre économique. Véritable " laboratoire ", la couche 4 du Petit Cloup Barrat permet de tester à la lumière d'une fouille récente la plupart des scénarios avancés pour expliquer cette variabilité synchronique des productions et des équipements (Langlais et Ducasse sous presse). S'il nous conduira à discuter du bien-fondé de cette bipartition " géo-culturelle », cet assemblage riche et varié associant industries lithiques, osseuses, parure et faune, s'inscrit dans un cadre analytique dépassant largement le Quercy : une fois décrites les principales composantes de la

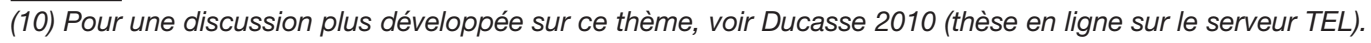


couche 4, nous verrons que les liens tissés nourrissent des problématiques interrégionales susceptibles d'enrichir nos connaissances sur les sociétés du DMG, tant en France qu'à l'échelle du sud-ouest européen.

\section{2 - Position stratigraphique, caractéristiques pédo-sédimentaires et limites de la couche 4 (HC, JCC et SD)}

Le secteur retenu pour la fouille est implanté perpendiculairement à un abri long de $8 \mathrm{~m}$ et profond de 4 $\mathrm{m}$, de part et d'autre du sondage 2003 (fig. 2A : bandes 7071-72). Au sein de cette grotte/abri, qui correspond à ce qui subsiste d'un réseau karstique superficiel que l'érosion verticale a fini par recouper pour former la doline (Castel et al. 2006 ; Chauvière et al. 2008), plusieurs unités sédimentaires ont pu être distinguées. Celles-ci ont été marquées par des processus divers (soutirage, ruissellement et gélifraction) généralement liés à l'évolution de ce modelé karstique, de son démantèlement jusqu'à son comblement. La majeure partie des remplissages clastiques, qui ont parfois été remaniés postérieurement (perturbations animales et humaines), provient ainsi du démantèlement des parois et de l'effondrement en blocs de la corniche du porche d'entrée.

\section{1 - La couche 4 au sein de la séquence du Petit Cloup Barrat}

Les observations menées depuis la campagne 2005 ont permis d'obtenir une vision claire et dynamique de cette stratigraphie complexe. Notre compréhension et, par conséquent, notre interprétation de la séquence s'est affinée à mesure que la fouille a progressé, nous conduisant à l'issue de la campagne 2008 à en proposer une synthèse détaillée (Camus 2009). L'ensemble se caractérise par une forte hétérogénéité inhérente au milieu karstique, l'analyse stratigraphique de la partie sommitale étant par ailleurs perturbée tant par la présence d'une fosse récente (fig. 3B) que par les remblais des fouilles anciennes (cf. supra). La séquence documentée à ce jour se compose, de la base au sommet, comme suit (fig. 3A) :

- l'ensemble $10^{11}$ traduit une ambiance karstique nette et inclut la couche $10 \mathrm{~N}$ dont le contenu archéologique pourrait être rapporté à une phase ancienne du Solutréen (Morala 2009) ;

- la couche 9 traduit une plus large ouverture et l'introduction de gélifracts. Elle contient un matériel archéologique très pauvre et actuellement non diagnostic ;

- l'ensemble 8 renferme à sa base (c.8a1) un sousensemble archéologique attribué au Solutréen supérieur (pointes à cran et feuille de saule). Lui succède la couche $8 a 2$ comportant des éléments lithiques diagnostics du
Badegoulien (raclettes et nucléus sur tranche à encoche). La couche 8c, en contact direct avec la couche 8a2, offre à son sommet un matériel tout à fait comparable à celui de la couche 4 (à ceci près que l'abri a pu la préserver des agents météoriques extérieurs). Les liens que la couche $8 \mathrm{c}$ entretient avec la 8d qui la surmonte sont encore discutés.

- l'ensemble 6/5 ainsi que la couche 7 indiquent plusieurs épisodes de ravinements. Les vestiges archéologiques y sont très rares et ne permettent pour le moment pas de diagnose chrono-culturelle;

- la couche 4, dont le matériel fait l'objet de cet article, correspond à une formation à matrice terreuse abondante. De nombreux blocs pluri-décimétriques à surface altérée y sont posés à plat, associés à des galets calcaires fracturés sur place. Ces gros éléments témoignent du recul du porche d'entrée. Le mur de la couche 4 est en légère pente vers le centre de la doline;

- les couches 3 à 0 ne renferment quant à elles pas d'ensembles archéologiques homogènes (rares vestiges du Magdalénien moyen mêlés à des tessons de céramique).

\section{2 - Définition des limites de la couche 4}

\subsection{1 - Limites verticales}

Essentiellement présente entre les travées $P$ et $S$ (un lambeau de couche existe au sud-ouest du carré T70 : fig. 3B), la couche 4 se caractérise par un contact stratigraphique diffus avec les ensembles sédimentaires qui l'encadrent (processus de remobilisation), rendant délicate la définition de ses limites verticales. Sur le terrain, la fréquence des éléments calcaires de grande taille a constitué l'un des critères de différenciation les plus pertinents. Mais ce n'est finalement qu'à partir des vestiges d'origine anthropique que ces limites ont pu être appréhendées plus précisément, une raréfaction nette du matériel en silex, des galets ainsi que de la faune, semblant signaler la base de la couche (fig. 4). Son sommet est plus difficile à définir, la transition c.3/c.4 se matérialisant, sans véritable discontinuité sédimentaire, par la présence de vestiges magdaléniens remobilisés (lamelles scalènes notamment) auxquels sont associés quelques tessons de céramique. L'ensemble de ce matériel, lithique ou osseux, se caractérise par une plus forte altération.

Afin de circonscrire au mieux l'étendue stratigraphique de la couche 4, nous avons entamé un travail d'analyse archéo-stratigraphique systématique ${ }^{12}$ dont les premiers résultats ont pu être inclus dans la présente publication. Pour chaque carré (PQRS-70 à 72), la distribution spatiale de l'ensemble des vestiges cotés (toutes catégories confondues) a permis d'établir une base de données réunissant les seuls éléments dont l'appartenance à une même « nappe » ne fait aucun doute. Dans la pratique, cela nous a conduits à :

(11) Le terme d'« ensemble » correspond à une séquence présentant plusieurs couches distinctes.

(12) Nous tenons à remercier F. Lacrampe-Cuyaubère pour son aide ainsi que ses conseils sur l'utilisation du logiciel Datadesk 6.0. 


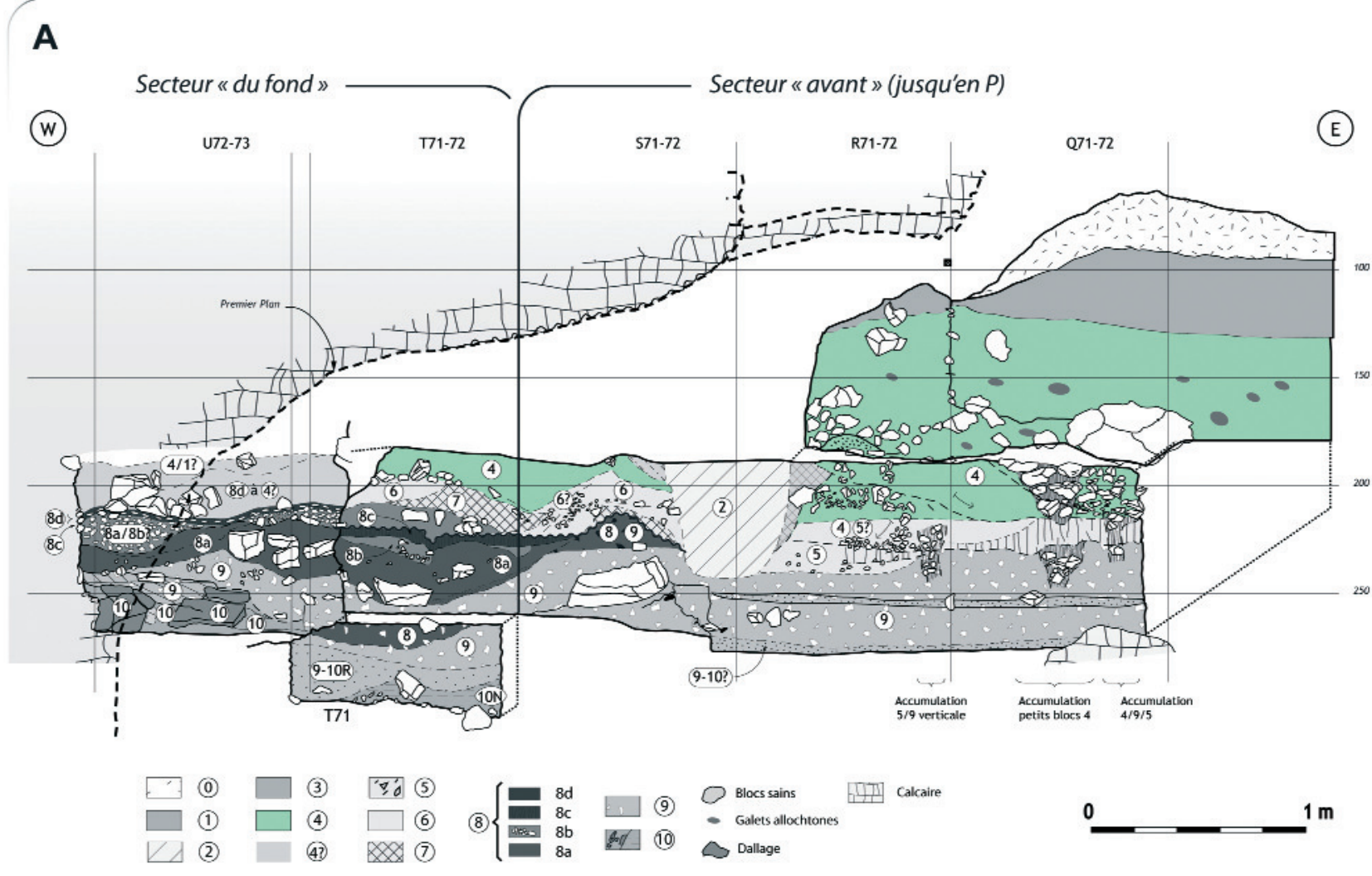

0 : Déblais

1 : Sol non compacté, traces de racines.

2: Remplissage anthropique dans une fosse.

3 : Dépôts à matrice marron très abondante,

rares clastes de taille inférieure à $5 \mathrm{~cm}$.

5 : Dépốt de gélifracts semi jointifs, taille 5 à $10 \mathrm{~cm}$ (probablement équivalent à 6).

6 : Dépôt à stratification horizontale de clastes grossiers, présence de blocs

(probablement équivalent à 5).

7 : Témoin d'une formation terreuse entre $5 / 6$ et 9

4: Dépôt de clastes émoussés/arrondis de 1 à $15 \mathrm{~cm}$ et blocs, plus ou moins jointifs dans une matrice marron à éléments

$1 \%$ allochtones (schistes, quartz, grès ferrugineux),

8 : Dépôts à niveaux colorés noirâtres et rouge (matière organique et bralés?) :

$8 \mathrm{a}$ : noirâtre $; 8 \mathrm{~b}$ : cailloutis : $8 \mathrm{c}$ : rougeâtre $: 8 \mathrm{~d}$ : indéfini entre 8 et 6 ou 4 ?

9 : Dépôts de gélifracts homométriques $(1$ à $5 \mathrm{~cm}$ ), à matrice pauvre

et clastes jointifs sans imbrication préférentielle.

$99 \%$ calcaires (gris à pâte fine et blanc biodétritique grossier), $\quad 10$ : Remplissage karstique interne remanié avec composante organique à sa base présence de quelques blocs allochtones (socle Massif Central).
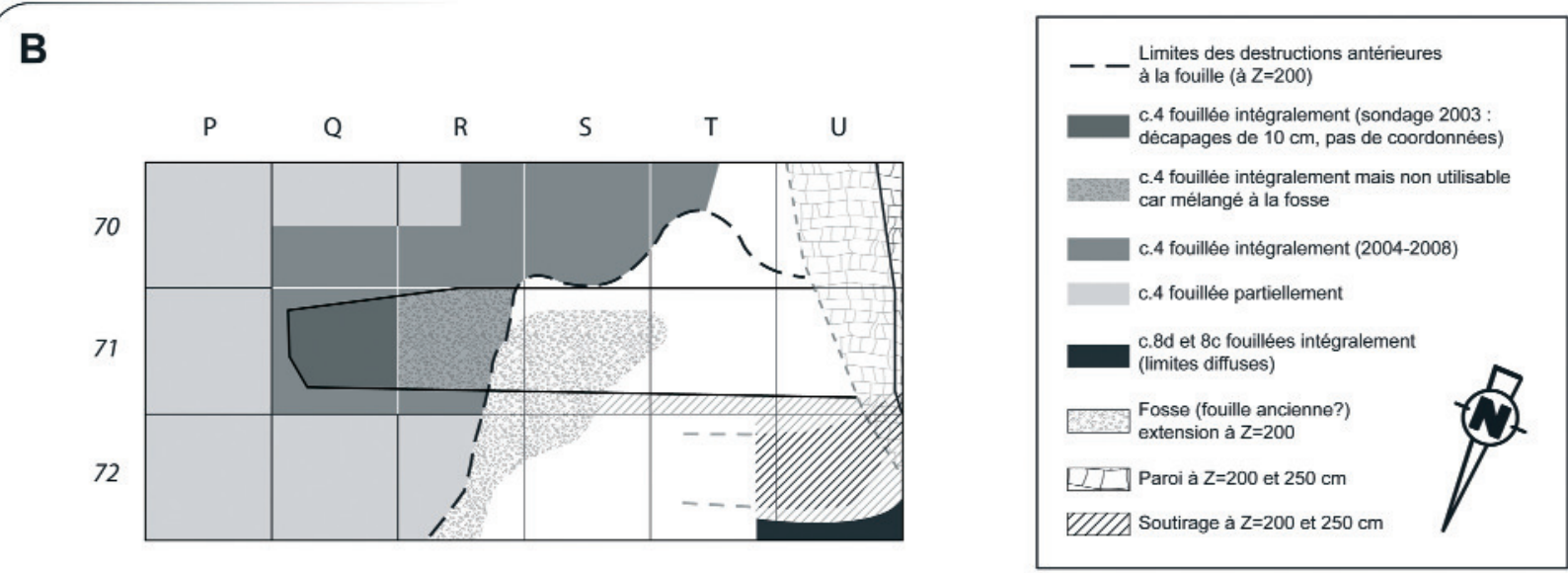

Figure 3 - A - Stratigraphie du Petit Cloup Barrat (Relevé et infographie Hubert Camus). B - Emprise de la fouille et degré d'avancement des travaux par quart de mètres-carrés.

Figure 3 - A - Stratigraphy of the Petit Cloup Barrat (Illustration by Hubert Camus). B - Extension of the excavations. 


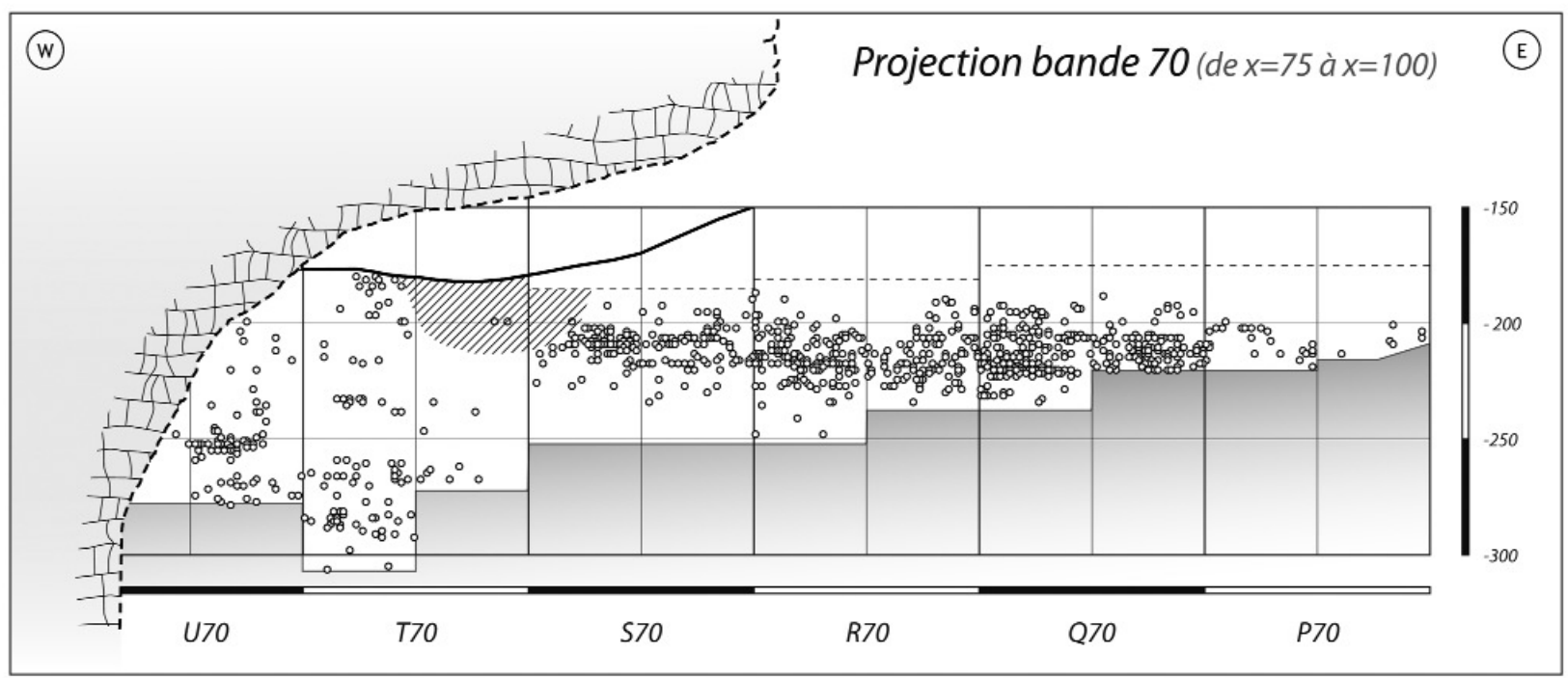

Limite des fouilles 2008

- - - - Limite supérieure de l'enregistrement systématique

PII/T Secteur perturbé (creusement antérieur aux fouilles)

Figure 4 - Projection sagittale de l'ensemble du matériel côté dans les deux secteurs (bande 70, X=75-100).

Figure 4 - Sagittal view of recorded remains in the two sectors (band $70, X=75-100 \mathrm{~cm}$ ).

1) occulter le matériel issu du sondage 2003 pour lequel les données contextuelles étaient insuffisantes (« tranches » de plusieurs dizaines de cm d'épaisseur) ; 2) extraire l'ensemble des éléments cotés et/ou inclus dans les décapages réalisés au sein des déblais (une partie du matériel 2004) ;

3) identifier les concentrations verticales de vestiges. En croisant cette approche avec les données chronoculturelles (cf. nature « diagnostique » de certains éléments lithiques et osseux), les premiers résultats ont pu être affinés ou nuancés, aboutissant in fine à une sorte « d'arrêt sur image " de la couche 4 à l'aube de la prochaine opération triennale (2011-2013). Bien que ce phénomène reste très marginal, la finesse des « tris " réalisés à partir des vestiges cotés s'accorde parfois mal avec le traitement des refus de tamis par décapage, l'échantillon considéré étant donc susceptible d'abriter quelques éléments intrusifs (cf. infra : le cas des lamelles scalènes). Les analyses présentées ci-dessous et, avant tout, les quantifications proposées, tiennent donc compte de ce découpage provisoire et « évolutif ».

\subsection{2 - Limites horizontales}

La couche 4 s'étend actuellement sur près de $9 \mathrm{~m}^{2}$ dont 3,5 seulement ont été intégralement fouillés. Au vu de l'emprise des « destructions " antérieures à la fouille (fouilles anciennes, fosse récente : fig. 3B), le sondage exploratoire réalisé en 2003 n'a concerné qu'une faible superficie de la couche 4 (moins de $2 \mathrm{~m}^{2}$ ) dont une partie est par ailleurs inutilisable compte tenu de son mélange avec le matériel de la fosse attenante. Quant au sondage effectué dans la bande 63 (P-Q-R : fig. 2A), non intégré à ce travail, il devra faire l'objet d'une nouvelle évaluation à la lueur des données acquises sur la couche 4, un niveau présentant de nombreuses similitudes sédimentaires ayant pu y être défini.

Le point le plus intéressant - et le plus discuté - concerne les relations entretenues entre la séquence définie à l'avant de l'abri et la succession des dépôts présents dans la partie couverte du gisement, seule zone à avoir à ce jour livré des éléments indiscutablement badegouliens $\left(\right.$ raclettes $\left.^{13}\right)$ et solutréens (pointes à cran à retouche couvrante et feuille de saule). Décrits en suivant, les vestiges caractéristiques de la couche 4 trouvent de réels parallèles dans le fond de l'abri et ce, malgré les divergences perçues dans les dynamiques pédosédimentaires. L'enjeu est de pouvoir situer les occupations de la couche 4 vis-à-vis des technocomplexes précédemment cités. Si ces éléments, présents entre les couches $8 \mathrm{c}$ et $8 \mathrm{~d}$, ne seront pas pris en compte lors des analyses présentées ici (ou à seul titre comparatif), ils n'en seront pas moins mobilisés lors d'une discussion globale, une approche archéo-stratigraphique (encore préliminaire compte tenu des faibles superficies fouillées dans ce secteur) complétée par la réalisation de quelques mesures d'âge C14 permettant d'apporter quelques éléments de compréhension (cf. infra).

(13) Notons qu'il existe quelques raclettes dans le secteur avant $(N=6)$, réparties entre les carrés $P 70, Q 70, Q 72$ et S70. Néanmoins, cinq d'entre elles, non cotées, proviennent des déblais des fouilles anciennes et se répartissent dans des intervalles d'altitudes bien supérieurs au niveau de la couche 4, parfois même mêlées à des fragments de céramique. La dernière, issue du carré R70, provient d'un décapage d'une dizaine de centimètres d'épaisseur ( $Z=199-209)$ associant déjà une lamelle scalène (Magdalénien moyen) aux éléments caractéristiques de la couche 4. 


\section{3 - Equipements et productions lithiques (ML et SD)}

Le gisement se partage donc en deux secteurs (fig. 3A) : la zone avant (PS70-72), qui permet notamment de suivre un véritable niveau conservé sur plusieurs mètres carrés, et le fond de l'abri (TU70-72) qui, de son côté, s'avère bien plus complexe puisqu'il livre sur un espace restreint une stratigraphie dilatée à la conservation inégale (cf. supra : dynamique de soutirage). Issues de l'analyse de la couche 4 , les données présentées ici seront, sur certains points particuliers, complétées par les informations collectées lors de l'étude de la couche $8 \mathrm{c} / \mathrm{d}$ dont le sommet livre des éléments typo-technologiques tout à fait comparables (cf. supra).

L'analyse litho-technologique des différentes roches hors silex reste à ce jour en suspens (Daulny 2005). Ainsi, bien que cet ensemble corresponde à un corpus quantitativement important, il ne jouera dans le cadre présent qu'un rôle secondaire. Une approche pétrographique a tout de même permis la mise en évidence d'une double composante, locale (quartzite, grès sidérolithiques) et allochtone (schistes, basaltes, granites et certains calcaires). Si une partie de ces éléments présente des stigmates témoignant de la confection d'un macro-outillage et de la mise en œuvre d'une production d'éclats, ce n'est qu'au prix d'une étude intégrale que les différents registres d'activités qui y sont liés pourront être véritablement identifiés et mis en relation avec les autres champs techniques. En attendant qu'un tel travail soit mené, notre intérêt s'est fixé sur les seuls vestiges lithiques en silex, témoins abondants qui, par leur originalité, tiennent une place centrale dans la définition de cet ensemble chrono-culturel (cf. infra).

\section{1 - Nature du corpus analysé}

Après redéfinition des limites inférieures et supérieures de la couche 4 (cf. supra), le matériel lithique pris en compte réunit 25555 objets en silex, dont près de $80 \%$ correspondent à des esquilles. Ces dernières $(\mathrm{N}=20294)$, qui résultent des multiples activités de débitage et de retouche, témoignent d'une bonne conservation gravimétrique des dépôts. Les 5261 pièces restantes se répartissent selon différents supports dont près de $15 \%$ sont transformés en outils (tabl. 1). En dehors des différents types d'éclat et fragments qui proviennent comme nous le verrons de chaînes opératoires diverses, la composante lamellaire (lamelles et éclats lamellaires), très abondante au sein des supports bruts $(43,8 \%$ de l'ensemble hors esquille), tient une place centrale dans l'équipement de chasse (selon l'observation de stigmates diagnostiques d'un fonctionnement en armatures : cf. infra). De leur côté, les lames et éclats laminaires, certes moins nombreux, jouent un rôle notable dans l'outillage de transformation (98 pièces, soit 34,6\% du total).

Malgré la diversité lithologique dont témoigne cet ensemble (étude en cours André Morala), le caractère préliminaire de cette approche au Petit Cloup Barrat ne permet actuellement pas d'alimenter une discussion approfondie d'ordre techno-économique. De fait, les paragraphes suivants s'attacheront à documenter l'assemblage de la couche 4 de manière globale, sans tenir compte de l'origine des matériaux exploités. Levier heuristique pour appréhender les assemblages lithiques du Paléolithique supérieur (Bon 2002 ; Tartar et al. 2006 ; Langlais 2007), la distinction " outillage domestique / équipement cynégétique » est ici adoptée.

\begin{tabular}{|c|c|c|c|c|c|c|c|c|c|}
\hline & \multirow{2}{*}{ Brut } & \multicolumn{5}{|c|}{ Outil } & \multirow{2}{*}{ Total } & \multirow{2}{*}{$\%$} & \multirow{2}{*}{$\%(1)$} \\
\hline & & microlithes & outils d'extrémité & outil latéraux & autres & percuteur & & & \\
\hline lame & 218 & 2 & 47 & 37 & 1 & & 305 & 1,19 & 5,80 \\
\hline éclat laminaire & 62 & & 8 & 4 & 1 & & 75 & 0,29 & 1,43 \\
\hline lamelle & 1239 & 391 & 10 & 9 & & & 1649 & 6,45 & 31,34 \\
\hline éclat lamellaire & 645 & 1 & 10 & 6 & & & 662 & 2,59 & 12,58 \\
\hline éclat & 1195 & & 33 & 38 & & & 1266 & 4,95 & 24,06 \\
\hline fragment & 1195 & & 25 & 18 & 36 & & 1274 & 4,99 & 24,22 \\
\hline casson & 21 & & & & & & 21 & 0,08 & 0,40 \\
\hline esquille & 20294 & & & & & & 20294 & 79,41 & \\
\hline rognon & 8 & & & & & 1 & 9 & 0,04 & 0,17 \\
\hline Total & 24877 & 394 & 133 & 112 & 38 & 1 & 25555 & & \\
\hline Total (1) & 4583 & & & 684 & & & 5261 & & \\
\hline
\end{tabular}

Tableau 1 - Supports et outils (outils d'extrémité : catégorie comprenant burins, grattoirs, grattoir-burin, perçoir-becs et lames appointées / outils latéraux : catégorie comprenant couteaux, lames, lamelles et éclats retouchés, pièces tronquées / (1): décomptes et proportions hors esquilles).

Table 1 - Blanks and tools (end tools : burins, end-scrapers, composite tools, perforators, pointed blades / side tools : retouched blades, bladelets and flakes, truncations / (1) : counts and proportions without "chips"). 


\section{2 - Quels objectifs économiques ?}

\subsection{1 - La composante " domestique »}

L'outillage à vocation domestique ${ }^{14}$ représente $5,4 \%$ de l'ensemble, soit 283 pièces (tabl. 2). S'il se compose de divers types, la catégorie dominante correspond aux pièces à retouches latérales $(\mathrm{N}=112)$. Celle-ci réunit de nombreux éclats et fragments d'éclats $(\mathrm{N}=63)$ parmi lesquels s'individualisent quelques éclats minces $(\mathrm{N}=11)$ tandis que les lames et éclats laminaires $\left(N=35\right.$, fig. $5 n^{\circ} 2$ et 8) présentent des retouches uni ou bilatérales plus ou moins profondes. Six pièces à « retouches » marginales et discontinues, sont définies comme " couteaux ». Deux racloirs sur éclats sont à noter (fig. 8, $n^{\circ} 6$ ).

Les burins, relativement nombreux ( $N=74$, soit $26 \%$ ), se déclinent sans préférence particulière selon trois soustypes : dièdre (fig. $5, n^{\circ} 1,3,5$ à 7 ), sur troncature $\left(n^{\circ} 4\right)$ et sur cassure (fig. $6 \mathrm{~B}, \mathrm{n}^{\circ} 10$ ). Ils sont pour plus de la moitié réalisés sur supports allongés ( $N=42$, soit $56 \%$ ), qu'il s'agisse de lames, d'éclats laminaires ou lamellaires, le reste des pièces ayant été confectionné sur éclats $(\mathrm{N}=32)$. Ce type d'outils est associé à de nombreuses chutes de

\begin{tabular}{|c|c|c|}
\hline & Nombre & $\%$ \\
\hline burin & 74 & 26,15 \\
\hline bec \& perçoir & 40 & 14,13 \\
\hline pièce esquillée & 20 & 7,07 \\
\hline grattoir & 10 & 3,53 \\
\hline encoche \& denticulé & 10 & 3,53 \\
\hline pièce tronquée & 8 & 2,83 \\
\hline grattoir-burin & 5 & 1,77 \\
\hline lame appointée & 4 & 1,41 \\
\hline Outils latéraux & 112 & \multirow{5}{*}{39,58} \\
\hline dont pièce retouchée & (80) & \\
\hline dont fgt de pièce retouchée & (24) & \\
\hline dont racloir & (2) & \\
\hline dont pièce d̀ retouches marginales & (6) & \\
\hline Total & 283 & \\
\hline
\end{tabular}

Tableau 2 - Décompte des outils à vocation domestique.

Table 2 - Count of « domestic » tools. burin $^{15}$. Généralement simples, les burins sont par deux fois associés à une seconde partie active (burin-bec), une extrémité tronquée s'y opposant dans un seul cas. En tant qu'outils composites, les premiers font écho à quelques grattoir-burin ( $N=5$, fig. $6 A, n^{\circ} 2$ et 3 ).

Les outils perforants, composés de 40 pièces, correspondent à la catégorie typologique la mieux représentée après celle des burins ( $14 \%$ de l'outillage). II s'agit de microperçoirs $(\mathrm{N}=20)$ en partie confectionnés sur lamelles et éclats lamellaires $(\mathrm{N}=12)$ ainsi que de becs et/ou perçoirs réalisés tant sur éclats et fragments d'éclats ( $N=12$ dont 1 perçoir multiple) que sur produits issus des débitages laminaires et lamellaires $(\mathrm{N}=8)$. Les pièces esquillées, qui sont, par définition, présentes sous la forme résiduelle de fragments d'éclats et d'une lame (fig. $8, n^{\circ} 7$ ), comptent pour $7 \%$ de l'ensemble. Le reste de l'équipement se partage entre grattoirs (dont deux portent un front ogival), pièces denticulées et à encoches, pièces tronquées mais aussi lames appointées ( $N=4)$. L'une d'entre elles, entière, se démarque nettement de l'assemblage (fig. 7). Présentant de nettes traces d'ocre sur ses deux faces, elle a été confectionnée à partir d'un support laminaire au profil régulièrement courbe et au talon facetté/abrasé témoignant de l'utilisation d'une percussion tendre organique. Cet outil en silex sénonien périgourdin faisait probablement partie d'un équipement de voyage au même titre que les quelques outils sur lame importés du Bergeracois où depuis les formations maastrichtiennes connues entre Chalosse et Armagnac (silex à Lepidorbitoïdes sp. : Normand 1986 ; Bon et al. 1996 ; Séronie-Vivien et al. 2006 ; Chalard et al. 2010 ; Colonge et al. 2011). L'identification d'un éclat de retouche en silex dit du «Grain de Mil » (Santonien de Charente-Maritime : Simonnet et coll. 2007 ; Caux 2011) nous renseigne là aussi sur la gestion d'un «stock » d'outils à longue durée de vie soulignant une certaine planification des activités.

Les outils sur lames présents dans la série recoupent donc deux réalités : l'apport de support transformés ou non, complété par une production réalisée in situ. De fait, malgré l'existence d'un nucléus prismatique abandonné au stade laminaire ${ }^{16}$, c'est essentiellement sur la base des produits et sous-produits du débitage que nous avons pu documenter cette composante d'un point de vue technologique.

La longueur initiale des supports reste difficile à déterminer compte tenu du caractère très fragmenté du corpus, fragmentation en partie due à une probable segmentation volontaire par percussion dont le rôle devra être éclairci $\left(\mathrm{N}=19\right.$, fig. $5, \mathrm{n}^{\circ} 10$ et fig. $6, \mathrm{n}^{\circ} 7$ ). Concernant leur gabarit,

(14) Constituant la sphère dite de « transformation », par opposition aux microlithes liés quant à eux à la sphère « d'acquisition ", cf. infra.

(15) Dans un certain nombre de cas il reste difficile de se prononcer sur l'origine technologique de ces supports à "pan revers » (63 pièces peuvent en effet provenir soit de l'entretien de l'outillage, soit d'un débitage sur tranche d'éclat ; modalité opératoire par ailleurs largement développée au sein de l'assemblage : cf. infra).

(16) Le faible nombre de nucléus pourrait d'ailleurs s'expliquer soit par une réduction continue des volumes vers des modules lamellaires, soit par une réorientation des objectifs en fin de course, privilégiant l'obtention d'éclats. La question de la représentativité de la zone fouillée peut également être posée $\left(9 \mathrm{~m}^{2}\right)$. 


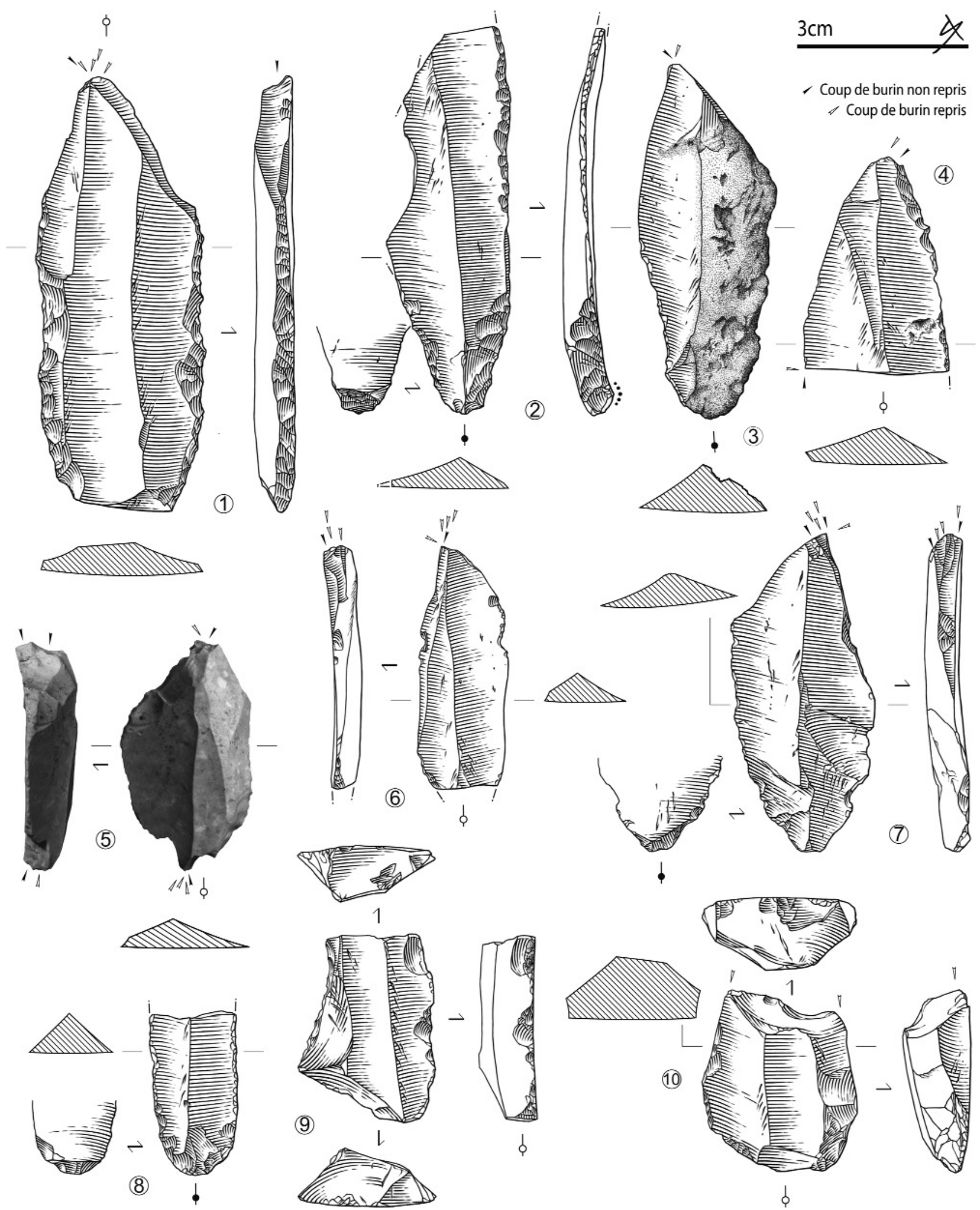

Figure 5 - Exemples d'outils sur lames - $n^{\circ} 1$ : burin dièdre sur lame retouchée, $n^{\circ} 2$ : lame à retouche proximale et latérale, $n^{\circ} 3$ à 7 : burins, $n^{\circ} 8$ : lame retouchée, $n^{\circ} 9:$ possible nucléus à lamelles sur tranche à encoche (fracturation distale par percussion), $n^{\circ} 10:$ lame retouchée et fracturée par percussion. Dessins et clichés S. Ducasse.

Figure 5 - Tools made on blades $-n^{\circ} 1$ : dihedral burin on retouched blade, $n^{\circ}$ 2: retouched blade, $n^{\circ} 3$ to $7:$ burins, $n^{\circ} 8$ : retouched blade, $n^{\circ}$ 9: bladelets core "sur tranche à encoche »? (distal fracturing made by percussion), $n^{\circ}$ 10: retouched blade broken by percussion. Drawings and pictures by S. Ducasse. 

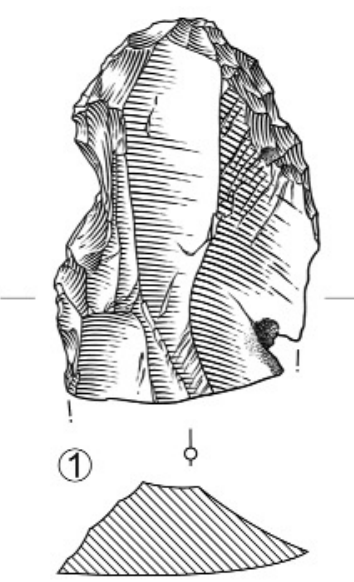

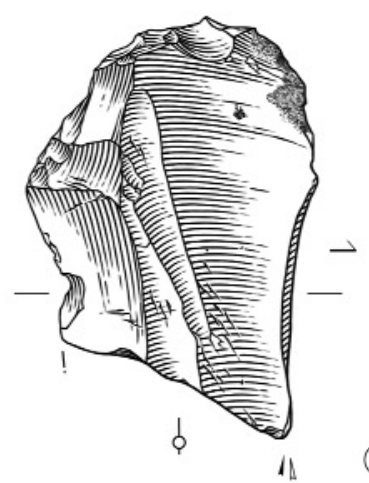

(2)
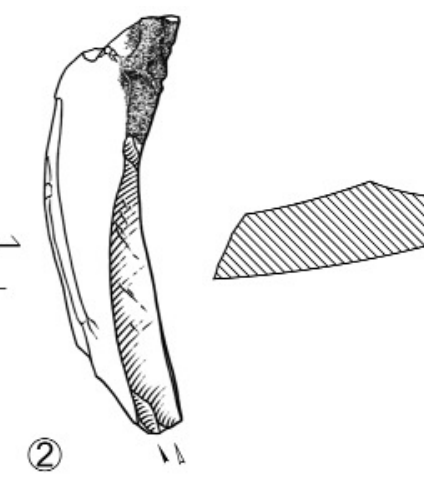

(3)
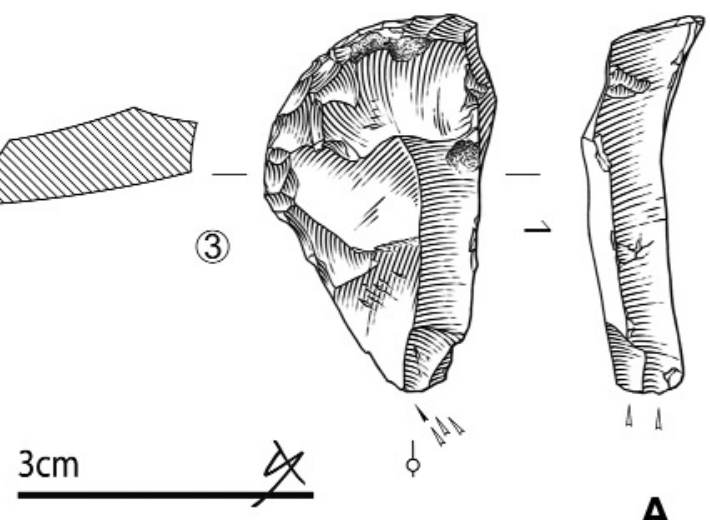

in

A I

A

- Coup de burin non repris

$\checkmark$ Coup de burin repris

B
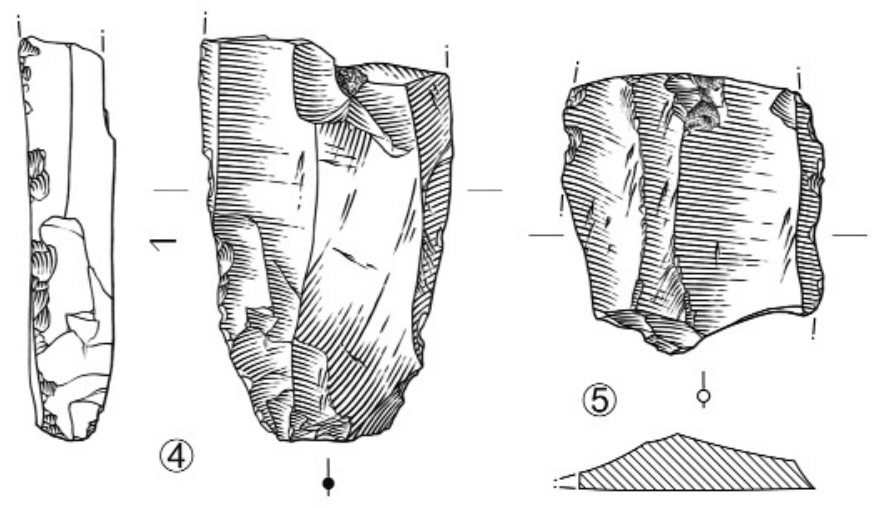

(5)
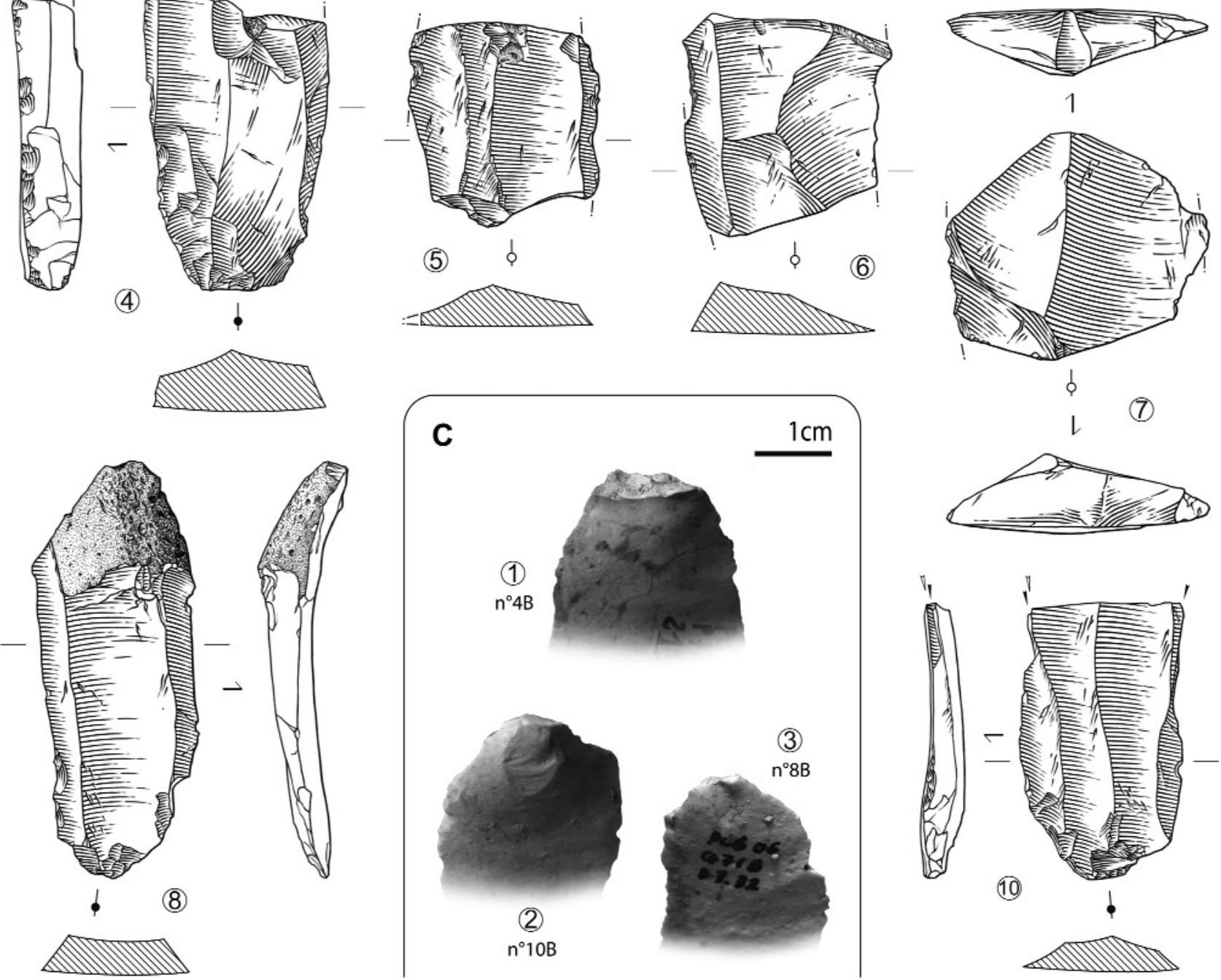

Figure 6 - Exemples de produits laminaires retouchés $\left(A-n^{\circ} 1\right.$ : grattoir, $n^{\circ} 2$ et 3 : grattoir-burins; $B n^{\circ} 4:$ lame retouchée, $n^{\circ} 10$ : burin) ou non ( $B-n^{\circ} 5$ à 8 ; le fragment $n^{\circ} 7$ présente les stigmates d'une fracturation par percussion). $C$ : détail de certains talons. Dessins et clichés S. Ducasse.

Figure 6 - Example of laminary blanks, retouched $\left(A-n^{\circ} 1\right.$ : end-scraper, $n^{\circ} 2$ and 3: end-scrapers/burins (composite tools); $B-n^{\circ} 4$ : retouched blade, $n^{\circ} 10$ : burin) or not $\left(B-n^{\circ} 5\right.$ to 8 ; fragment $n^{\circ} 7$ shows stigmata of fracturing by percussion). $C$ : details of few butts. Drawings and pictures by $S$. Ducasse. 


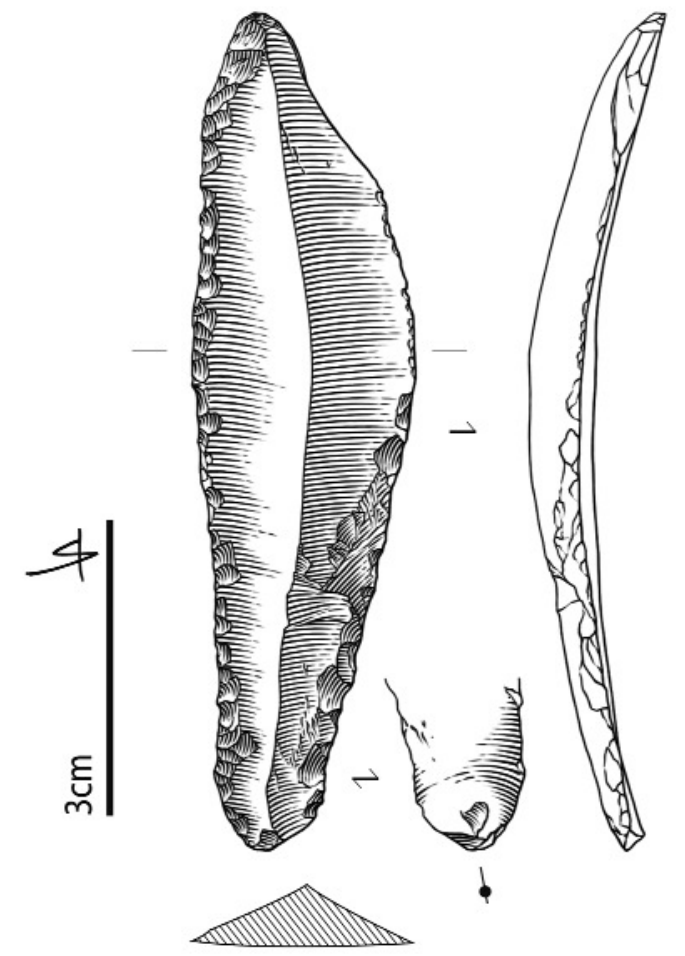

Figure 7 - Lame appointée, retouchée et ocrée en silex sénonien. Dessin S. Ducasse.

Figure 7 - Pointed and retouched blade that shows traces of ocher (senonian flint). Drawing S. Ducasse.

les lames présentent des largeurs comprises entre 10 et $30 \mathrm{~mm}$ pour une épaisseur située entre 2 et $6 \mathrm{~mm}$ sans véritable discontinuité avec les lamelles (le seuil lamelamelle se situe ici autour de $10 \mathrm{~mm}$ de largeur : graph. 1). L'examen des talons associés aux supports de plein débitage montre l'emploi préférentiel d'un percuteur tendre organique (fig. $5, n^{\circ} 2,7$ et 8 , fig. $6 C, n^{\circ} 1$ ) sur des corniches abattues par simple abrasion. Certains produits se distinguent néanmoins par une préparation plus soignée (facettage des plans de frappe : $N=5$ ). De leur côté, les éclats laminaires portent le plus souvent les stigmates d'une percussion minérale employée selon un geste plus rentrant. La lecture des faces supérieures indique quant à elle la mise en œuvre d'un débitage de type « semi-tournant » comme en témoigne au demeurant l'unique nucléus analysé. Le débitage semble essentiellement unipolaire, les quelques produits bipolaires devant être interprétés comme les indices d'une correction distale de la table effectuée à l'aide de courts enlèvements opposés. Quelques pièces à crête ou néocrête $(\mathrm{N}=13)$ témoignent d'aménagements transversaux destinés à maintenir des convexités favorables. Enfin, si l'existence de rares lames à pan revers pourrait indiquer la conduite d'un débitage laminaire « sur tranche » comme elle existe par exemple au sein de la séquence inférieure de l'abri Gandil (Langlais 2010, p. 70-71), l'éventualité que ces supports puissent s'intégrer dans les premières séquences d'initialisation lamellaire ne doit pas être négligée (cf. infra).

Une part des éclats alimentant l'équipement de transformation provient des chaînes opératoires laminaires et/ou lamellaires (5 outils sont par exemple confectionnés sur tablettes de ravivage). Par ailleurs, une production autonome d'éclats épais, essentiellement mise en œuvre afin d'obtenir des supports de nucléus à lamelles $(62 \%$ des nucléus : cf. infra), a généré des sous-produits qui, pour certains, ont pu être récupérés comme supports d'appoint $(5,6 \%$ des éclats sensu lato ont été transformés en outils domestiques). Au contraire, utilisés préférentiellement comme outils latéraux retouchés ou non (racloirs, couteaux ?), des éclats minces $(\mathrm{N}=41)$ sont obtenus à partir d'un débitage autonome lié à l'exploitation de galets (ou portion de galets) de silex local. Menée ici au percuteur dur, cette production, commune au Magdalénien inférieur et au Badegoulien (Morala 1993 ; Cazals 2000 ; Cretin 2000 ; Bracco et al. 2003 ; Langlais 2007 ; Ducasse 2010), permet l'obtention de supports minces lors de séquences courtes, la recherche d'une superposition des enlèvements générant parfois des produits concaves très caractéristiques (fig. 8, $n^{\circ} 2$ et 3). Bien que la répétition de ces courtes séries puisse progressivement envahir la totalité de la surface du galet, il semble qu'au sein de la couche 4 cette production n'ait été menée que de manière ponctuelle, les rares nucléus associés $(\mathrm{N}=4)$ témoignant d'une faible productivité (peu de négatifs, larges plages néocorticales (fig. $8, n^{\circ} 1$ ).

Si les lamelles et éclats lamellaires sont, dans de rares cas, intégrés à l'équipement domestique, leur rôle économique est prioritairement lié à la confection d'armatures composites : ce besoin, nettement perceptible à travers l'abondance de microlithes (394 pièces, soit près de $60 \%$ du total des supports retouchés), a donc motivé la mise en œuvre d'une importante production lamellaire intégrée à la sphère cynégétique.

\subsection{2 - La composante cynégétique}

Le groupe des microlithes témoigne d'une relative diversité typologique (tabl. 3). En dehors des ébauches, cet ensemble regroupe 375 pièces parmi lesquelles les lamelles à dos simples sont les mieux représentées $(\mathrm{N}=261)$. Bien que de nombreux fragments soient réunis sous cette appellation par défaut d'extrémité, une part de ces pièces correspond à des morphotypes « neutres " qu'une analyse de détail, en cours, permettra sans doute de mieux caractériser. Viennent ensuite les lamelles et microlamelles à dos dextre marginal $(\mathrm{N}=64)$ qui singularisent cet assemblage, complétées par quelques microlamelles à dos inverse ainsi qu'une diversité de sous-types : lamelles à dos inverse, alterne, tronquées, denticulées voire appointées. Malgré leur rareté $(\mathrm{N}=7)$, les lamelles à dos dextre étroites, parfois appointées, constituent une autre particularité de ce corpus. Notons que si les 10 lamelles scalènes recensées doivent être considérées comme intrusives, cela ne remet pour autant pas en cause l'intégrité de la série analysée. Cette " intrusion » résulte en effet de l'échantillonnage auquel nous avons procédé, incluant certains décapages de plus 


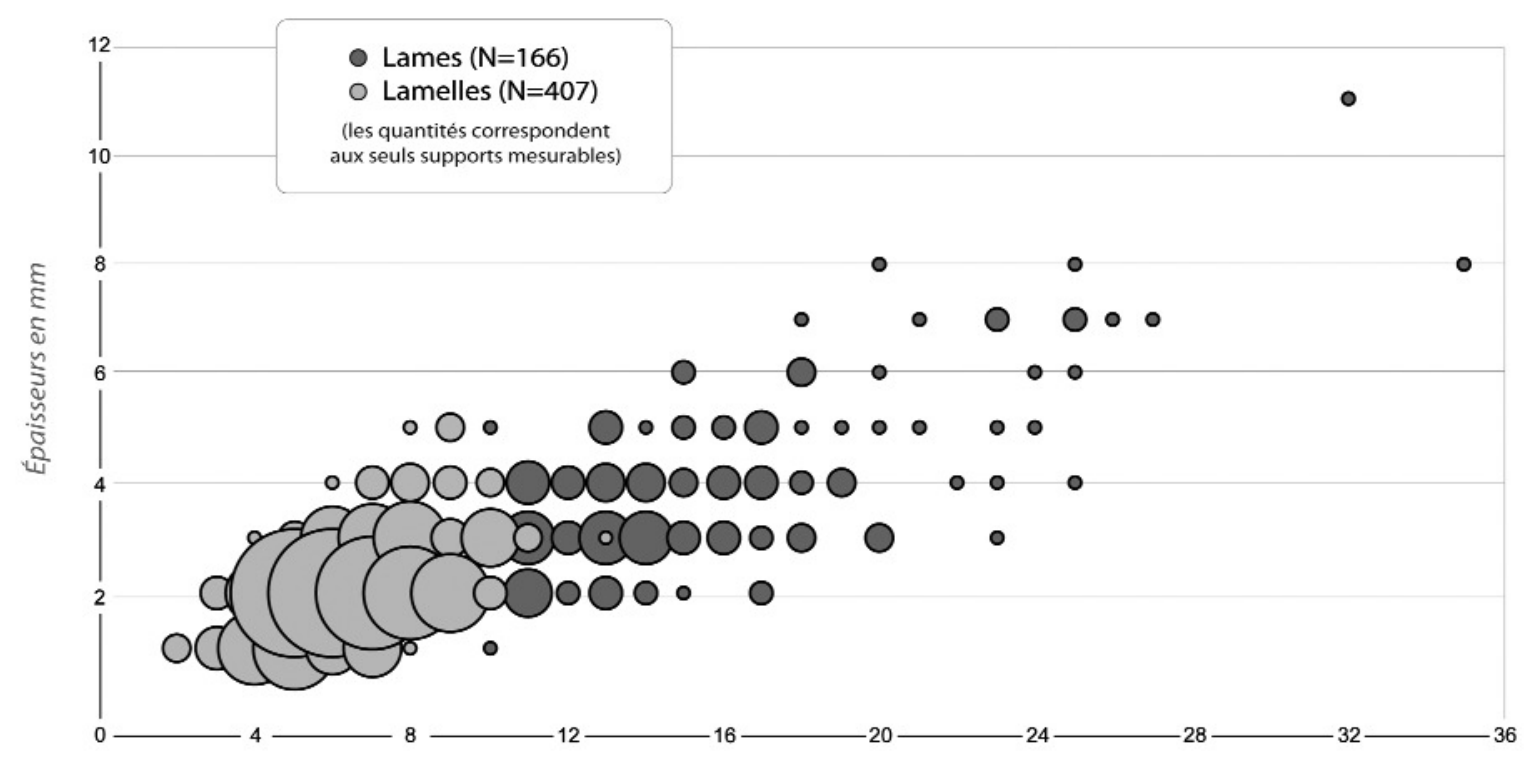

Largeurs en $\mathrm{mm}$

Graphique 1 - Gabarits des lames et lamelles brutes mesurables ( $N$ total $=573$ ).

Graphe 1 - Dimensions of mesurables blades and bladelets $(N$ total $=573$ )

\begin{tabular}{|c|c|c|}
\hline & Nombre & $\%$ \\
\hline LD simple & $\begin{array}{l}278 \\
(17)\end{array}$ & 70,56 \\
\hline $\begin{array}{l}\text { LD et MLD dextre marginal } \\
\text { dont ébauche }\end{array}$ & $\begin{array}{l}66 \\
(2)\end{array}$ & 16,75 \\
\hline LD dextre étroite & 7 & 1,78 \\
\hline MLD & 8 & 2,03 \\
\hline LD senestre marginal & 4 & 1,02 \\
\hline LD inverse & 4 & 1,02 \\
\hline LD alterne & 1 & 0,25 \\
\hline LD alterne marginal & 1 & 0,25 \\
\hline LD tronquée & 5 & 1,27 \\
\hline LD denticulée & 4 & 1,02 \\
\hline LD appointée & 2 & 0,51 \\
\hline LDD appointée & 1 & 0,25 \\
\hline $\mathrm{PD}$ & 3 & 0,76 \\
\hline Lamelle scalène & 10 & 2,54 \\
\hline Total & 394 & \\
\hline
\end{tabular}

Tableau 3 -Typologie des microlithes ( $L D$ : lamelle à dos / MLD : microlamelle à dos / LDD : lamelle à dos double / PD : pointe à dos).

Table 3 - Microliths typology (LD : backed bladelet / MLD backed microbladelet / LDD : double backed bladelet / backed point). de $10 \mathrm{~cm}$ réalisés à l'interface entre la couche 4 et l'ensemble supérieur attribué au Magdalénien moyen (cf. supra). La projection verticale comparée des lamelles scalènes et des lamelles à dos dextre marginal (fig 9) confirme d'ailleurs l'improbabilité de leur association ${ }^{17}$

\section{Un morphotype original : les lamelles et microlamelles à dos dextre marginal}

Isolé dès la campagne 2003 au sein d'un matériel remanié (diagnostic AM, Langlais et al. 2004), ce type de microlithe a, par son abondance ainsi que son originalité, été très tôt considéré comme un élément marquant de la série récoltée depuis 2004 en couche 4 (fig. 10A). Ceci nous a du même coup permis de tisser des liens archéologiques entre les deux secteurs de la fouille, pourtant actuellement distingués sur des critères de dynamique sédimentaire (cf. supra). Ce « marqueur » typo-technologique, qui participe à une discussion plus large sur la nature des équipements cynégétiques du DMG (cf. infra), se rencontre également au sommet de la couche 8c (U72, T71-72). Aux 64 pièces décomptées en couche 4 (tabl. 3) s'ajoutent 81 exemplaires extraits à la fois du fond de l'abri mais aussi des diverses zones remaniées (fosse en RS-71 72).

La silhouette de ces lamelles, accentuée par les divers aménagements réalisés après débitage, est le caractère qui fonde cette originalité : il s'agit d'un support mince, dissymétrique et le plus souvent appointé naturellement et montrant une torsion dextrogyre (ou contra-horaire : Pelegrin et O'Farrel 2005) plus ou moins marquée (fig. 10A,

(17) L'hypothèse alternative d'un faciès de transition (Cazals et Langlais 2006) nous semble actuellement difficile à argumenter. Rappelons cependant la présence de quelques lamelles à dos et troncature oblique à Lascaux (Allain 1979) et à Reverdit (Bourdier et al. sous presse). 

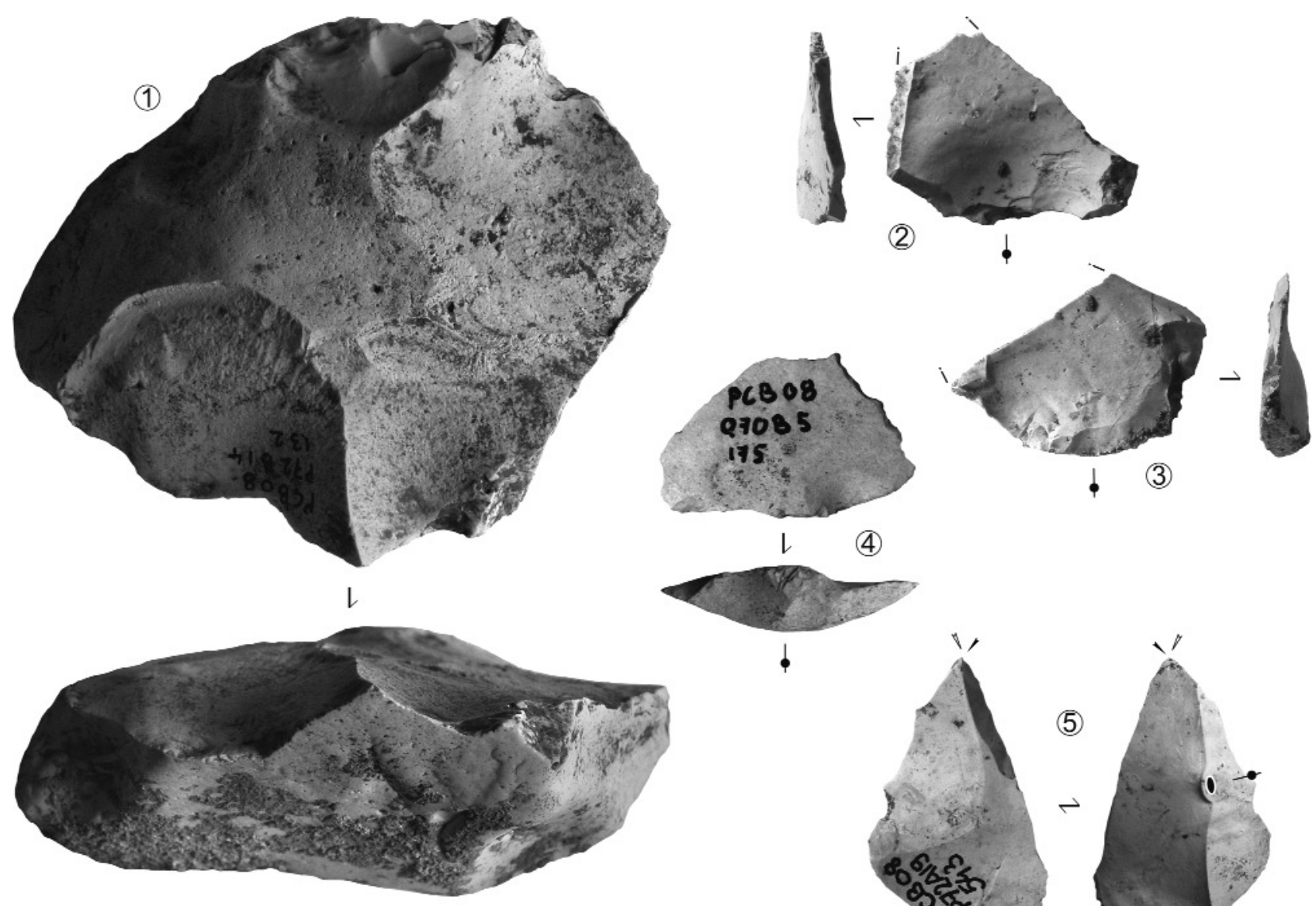

(4)
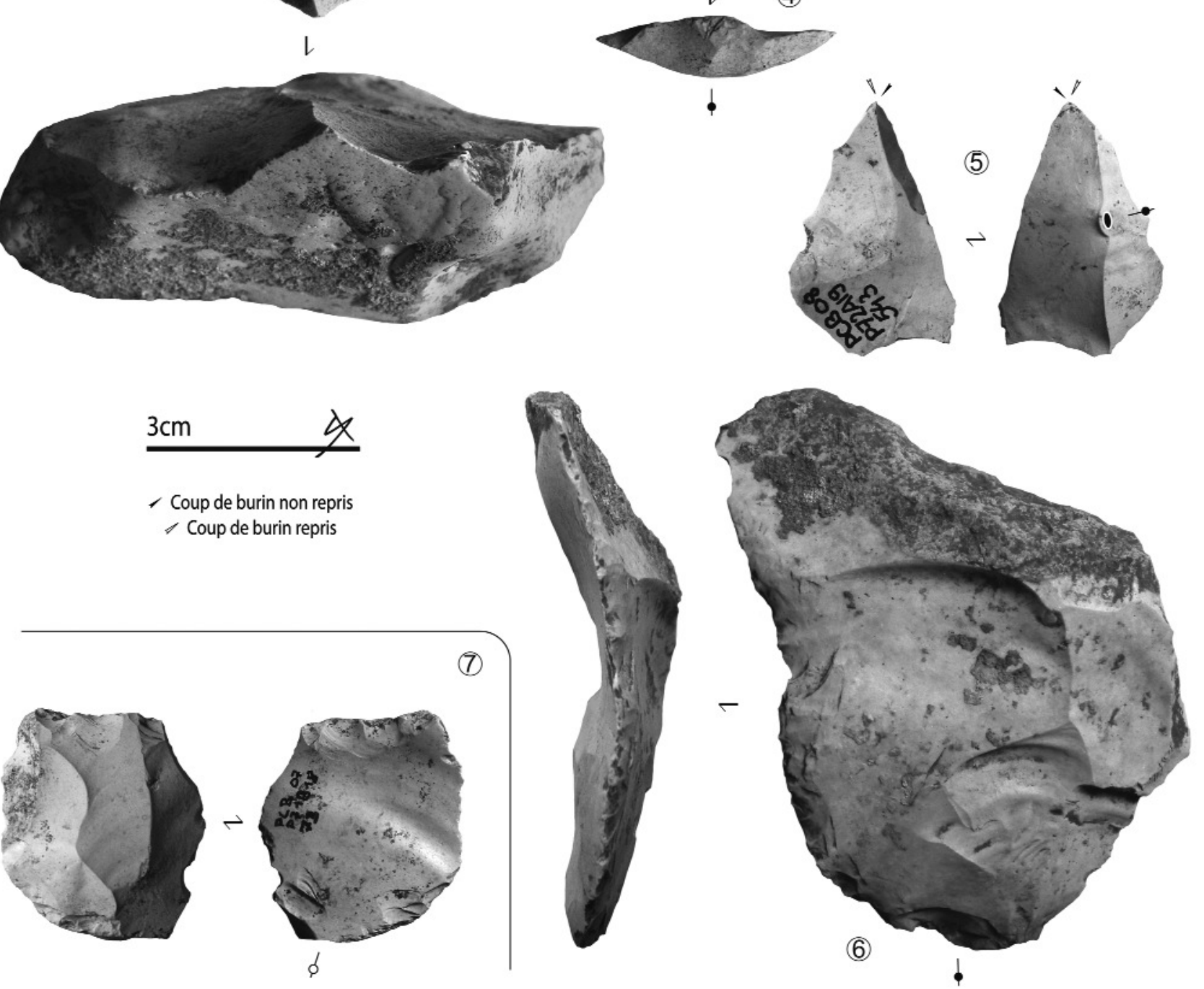

Figure 8 - Une production autonome d'éclats ( $n^{\circ} 1$ : nucléus à éclats minces, $n^{\circ} 2$ et 3 : éclats minces, $n^{\circ} 4:$ perçoir, $n^{\circ} 5:$ burin, $n^{\circ} 6$ : racloir sur grand éclat); $n^{\circ} 7$ : pièce esquillée. Clichés $S$. Ducasse.

Figure 8 - Autonomous flake production ( $n^{\circ} 1$ : core that produces thin flakes, $n^{\circ} 2$ and 3: thin flakes, $n^{\circ} 4$ : perforator, $n^{\circ} 5$ : burin, $n^{\circ} 6$ : side-scraper) ; $n^{\circ}$ 7: "pièce esquillée ». Pictures S. Ducasse. 


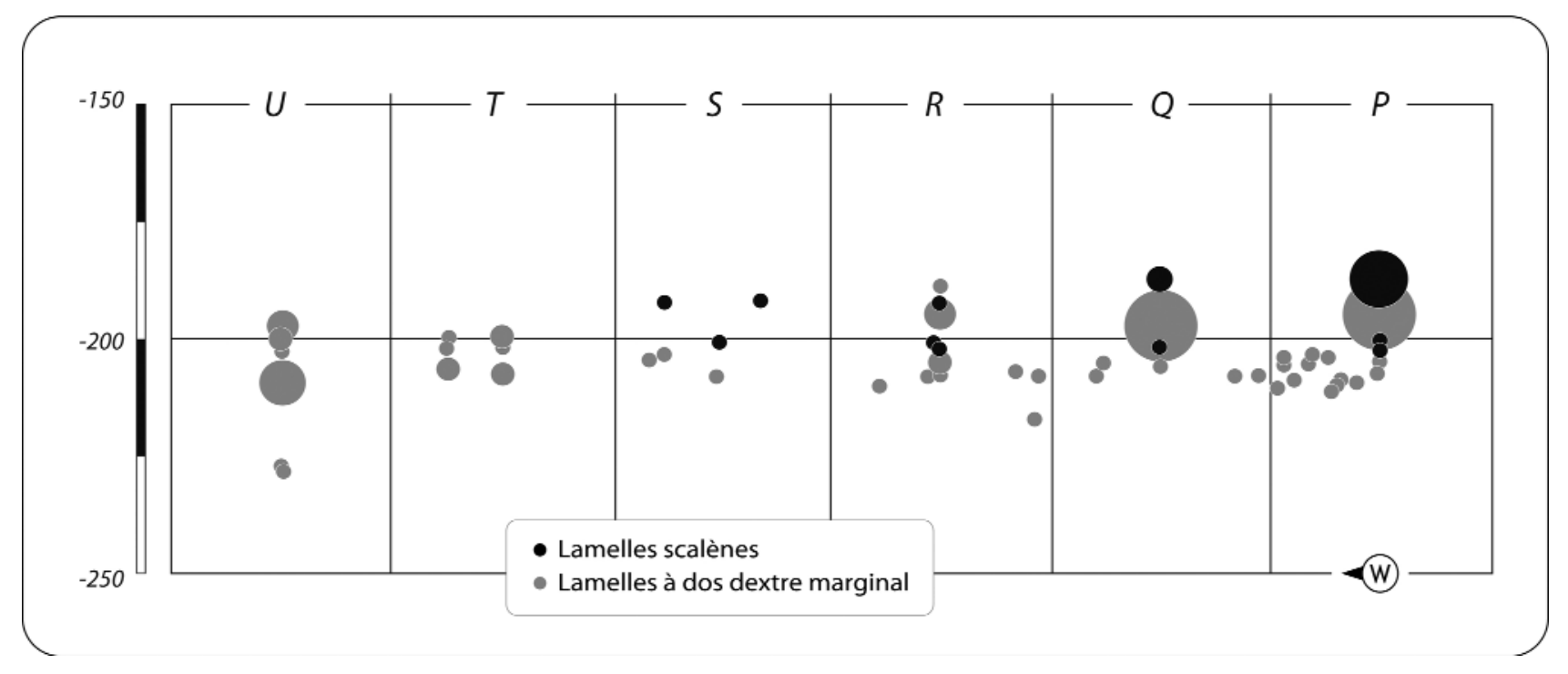

Figure 9 - Projection comparée des lamelles scalènes et des lamelles à dos dextre marginal (pièces cotées et tamis).

Figure 9 - Compared view of stratigraphic distribution of «lamelles scalènes » and bladelet with right- hand and marginal back.

$\mathrm{n}^{\circ} 4,9,11$ ou 12). Cette dissymétrie, définie par la convergence distale d'un bord droit rectiligne opposé à un bord gauche convexe, est appuyée à la fois par le façonnage d'un dos dextre, mais aussi par un bordage proximal et/ou distal venant conformer la délinéation du tranchant sénestre. Le dos, réalisé par une retouche directe, présente divers degrés d'abattement (Christensen et Valentin 2004, fig. 104, p.122) : si de très rares exemplaires montrent des dos " envahissants ", ce sont, par définition, les bords minces abattus par une retouche marginale qui dominent. Dans certains cas, cette modification du tranchant s'apparente à un simple égrisage (fig. $10 \mathrm{~A}, \mathrm{n}^{\circ} 13$ ou 14). Dans la mesure où 11 pièces portent des stigmates diagnostiques d'un impact apical violent (enlèvements de type burinant : fig. $\left.10 \mathrm{~A}, \mathrm{n}^{\circ} 18\right)$, l'hypothèse d'une utilisation de ces pièces comme armatures de trait est actuellement privilégiée (hypothèse d'ailleurs testée par des montages et tirs expérimentaux : Pétillon et al. 2011)

Si la mesure de longueur des pièces (sub-)entières montre deux concentrations autour de $14-16 \mathrm{~mm}$ et $23-25 \mathrm{~mm}$ (fig. 10B, à droite), un certain étalement peut être perçu, définissant un continuum dimensionnel au sein d'un même morphotype comme c'est notamment le cas à SaintGermain-La Rivière (Langlais 2010, p. 107 et 274). Le rapport largeur/épaisseur révèle de son côté une forte normalisation des gabarits autour de 3-5 mm de large pour 1-2 mm d'épaisseur (fig. 10B, à gauche). L'origine technologique de ces lamelles et microlamelles dextrogyres, nettement prédéterminées (faible modification des gabarits par la retouche), est probablement double. Les lamelles sont produites à partir d'un schéma opératoire basé sur l'installation et la gestion d'une surface de débitage dissymétrique : un flanc parallèle à l'axe longitudinal de la table est opposé à un flanc convergent vers la base du nucléus, cette dissymétrie favorisant la recherche de supports tors (fig. 11 à 13). Des micro-enlèvements proximaux, visibles tant sur les nucléus que sur les produits recherchés, permettent de décentrer la nervure-guide afin d'assurer la torsion des supports ainsi débités. L'entretien de cette dissymétrie et/ou de cette convergence distale passe par l'extraction d'éclats lamellaires fronto-latéraux unipolaires. Quand les conditions le permettent, plusieurs lamelles dextrogyres peuvent être extraites en série. Réalisée à partir de cassons, rognons ou éclats épais, cette production est généralement soignée, les talons, préférentiellement lisses abrasés ou punctiformes, témoignant de l'emploi fréquent d'un percuteur tendre (organique et/ou minéral) utilisé selon un geste tangentiel. Les supports microlamellaires de même silhouette (fig. 14A) ont été obtenus soit par réduction des tables lamellaires (cette réduction pouvant alors expliquer le faible nombre de nucléus répondant au schéma opératoire précédent : $\mathrm{N}=4$ ), soit à partir de productions autonomes « sur tranche » ou « sur front ventral déjeté » (cf. infra : fig. 15 et 16). Soupçonnée, bien que très théorique, la piste d'une progression continue du débitage marqué par la succession, sur un même volume, de microlamelles puis de lamelles dans le cadre d'une exploitation sur «tranche d'éclat » (fig. $12, n^{\circ} 4$ et 5) devra être testée lors de la poursuite des analyses (par le biais de remontages notamment). Quoi qu'il en soit, la complémentarité de ces deux objectifs est manifeste sur certains nucléus « mixtes » associant deux tables distinctes répondant à chacune de ces intentions (fig. $12, n^{\circ} 1$ et 4 ). 


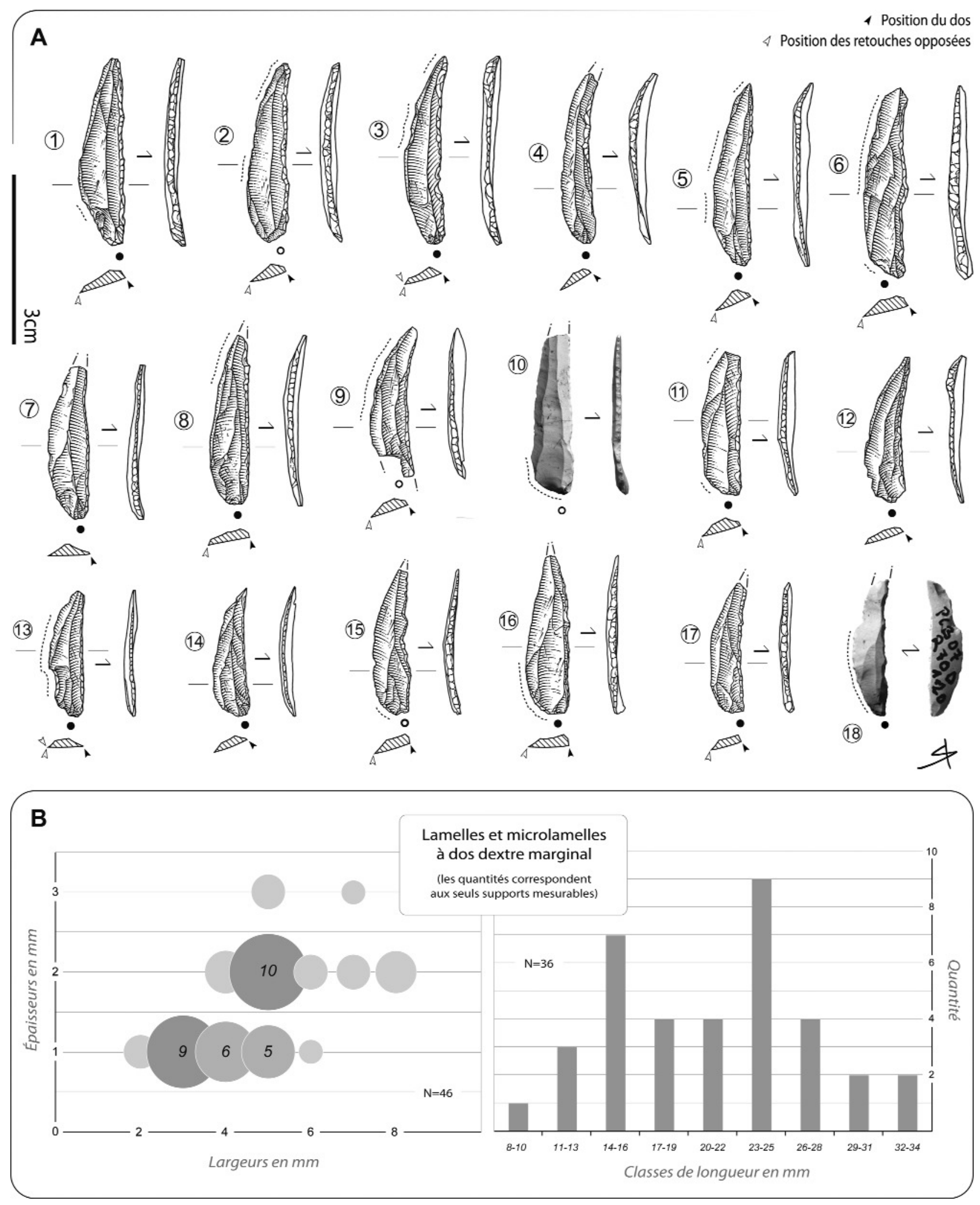

Figure 10 - A - Exemples de lamelles à dos dextre marginal des couches 4 et $8 c$. B - à gauche : gabarits des lamelles et microlamelles à dos dextre marginal $(\mathrm{N}=46)$, à droite : histogramme de longueur des lamelles et microlamelles à dos dextre marginal ( $N=36)$. Dessins S. Ducasse.

Figure 10 - A - Examples of bladelets with right-hand and marginal back from layer 4 and 8c. B - on the left side: width and thickness $(N=46)$, on the right side : length histogram $(N=36)$. Drawings $S$. Ducasse. 


\begin{tabular}{|c|c|c|c|c|c|}
\hline & \multicolumn{4}{|c|}{ Supports } & \multirow{2}{*}{ Total } \\
\hline & lamelle & éclat lamellaire & éclat & fragment & \\
\hline pan revers & 166 & 125 & 1 & 4 & 296 \\
\hline pan revers/CdB & 44 & 5 & & & 49 \\
\hline crête & 2 & & & & 2 \\
\hline néocrête & 16 & 11 & 5 & 2 & 34 \\
\hline $\begin{array}{l}\text { néocrête sur tranche } \\
\text { dont à encoche }\end{array}$ & 24 & $\begin{array}{l}22 \\
(1)\end{array}$ & & 1 & 47 \\
\hline néocrête sur tranche/CdB & 11 & 3 & & & 14 \\
\hline sous-crête & 5 & & & & 5 \\
\hline fronto-latéral & & 14 & & & 14 \\
\hline cintrage & & 60 & 6 & 2 & 68 \\
\hline dont sur tranche & $\begin{array}{c}1 \\
(1)\end{array}$ & & $\begin{array}{l}44 \\
(1)\end{array}$ & 2 & 47 \\
\hline Total & 269 & 240 & 56 & 11 & 576 \\
\hline
\end{tabular}

Tableau 4 - Caractéristiques techniques des lamelles, éclats lamellaires, éclats et fragments $(C d B$ : chute de burin).

Table 4 - Technical attributes of bladelets, lamellar flakes, flakes and fragments ( $C d B$ : burin spall)

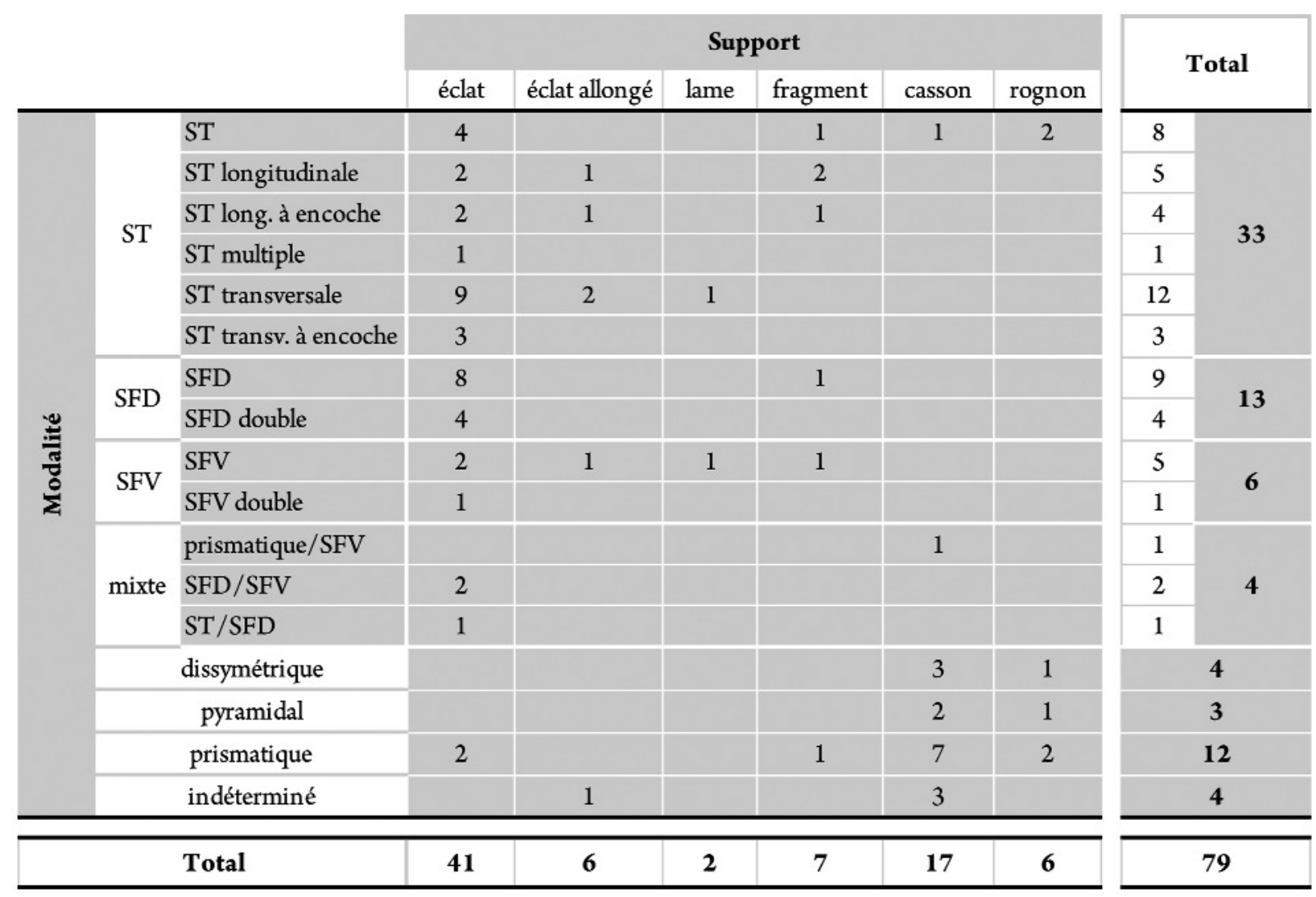

Tableau 5 - Les différentes modalités de production (micro-)lamellaires (ST : sur tranche / SFD : sur front dorsal / SFV : sur front ventral).

Table 5 - Types of bladelets and microbladelets productions 


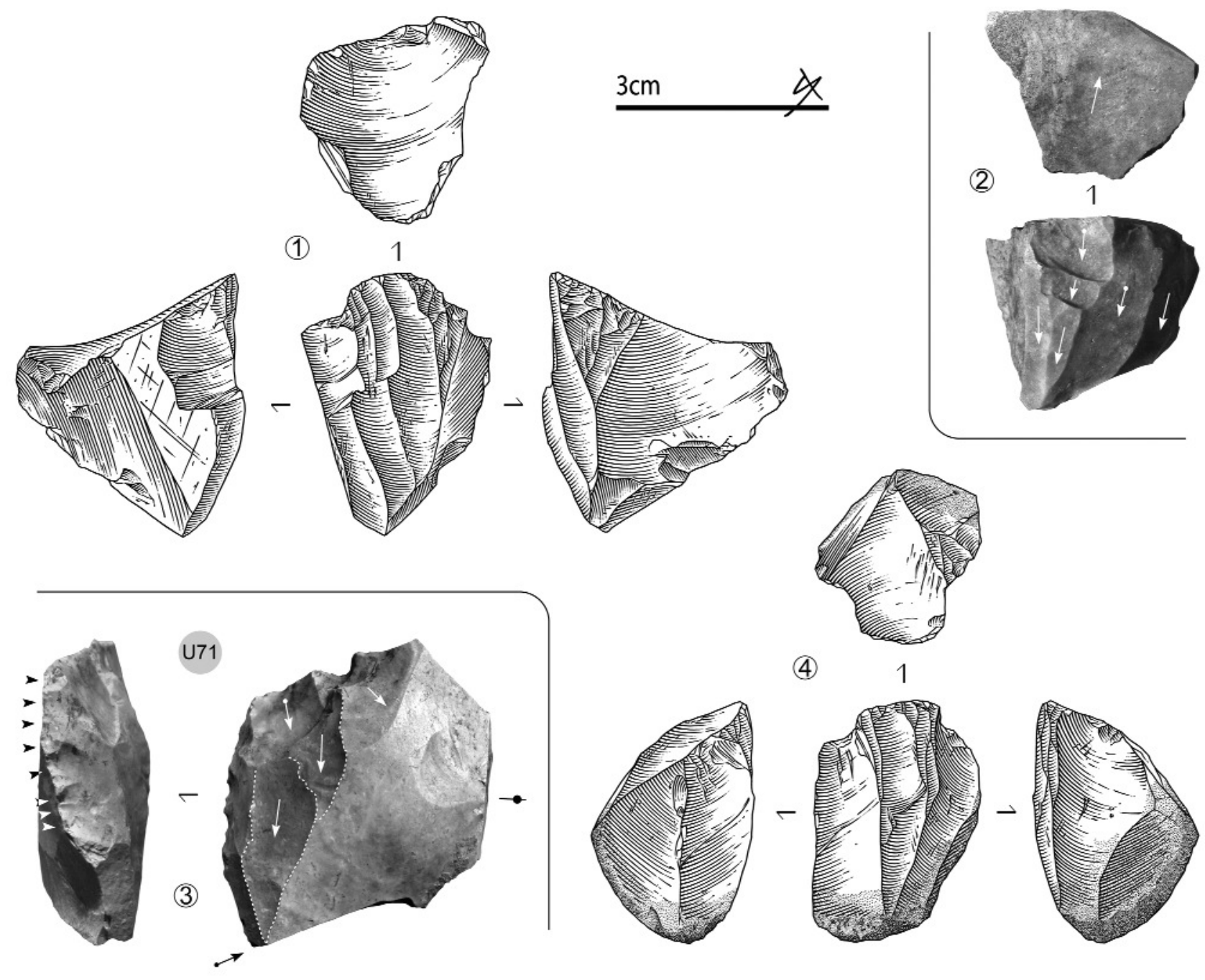

Figure 11 - Une production de supports dissymétriques et convergents ( $n^{\circ} 2$ et 3 : nucléus issus de la campagne 2003). Notons que la pièce $n^{\circ} 3$, correspondant à un débitage lamellaire sur tranche d'éclat, partage à première vue quelques caractères techniques avec les débitages " plans, préférentiels » (ex. "pièces de la Bertonne/d'Orville »: Ducasse et Langlais 2008, Chehmana /et al./2009). La " troncature inverse » si caractéristique de ces productions semble tenir ici un rôle distinct (cf. texte). Dessins et clichés S. Ducasse.

Figure 11 - asymmetric and convergent blanks production ( $n^{\circ} 2$ and 3: cores from 2003 field operation). Note that object $n 3$, despite appearances, do not fit with "Bertonne and Orville cores " (Ducasse and Langlais 2008, Chehmana et al. 2009). Here, the inverse "truncation" does not play the same technical role (see discussion in the text). Drawings and pictures S. Ducasse.

Une diversité des modalités de débitage micro-lamellaire ${ }^{18}$

L'une des caractéristiques de l'industrie de la couche 4 réside donc non seulement dans une forte variabilité des types de microlithes mais aussi dans une grande diversité des modalités de débitage (micro-)lamellaires associés. Tandis que la recherche de grands supports rectilignes s'accorde parfois avec l'exploitation de nucléus sur rognons aux architectures prismatiques et/ou pyramidales, des chaînes opératoires ramifiées sont mises en œuvre afin de produire différents types de supports : en dehors de certaines lamelles dextrogyres, l'installation de tables lamellaires et microlamellaires sur la tranche ou dans l'épaisseur d'éclats épais nous conduit à définir plusieurs modalités de production distinctes (tabl. 5, cf. note 15) :

Lorsque ces volumes sont exploités dans leur épaisseur, deux types de modalités dites sur « front » d'éclat peuvent être définis. La première, baptisée "sur front dorsal ", correspond à la recherche de lamelles et de microlamelles

(18) La terminologie technique employée dans le cadre de cette contribution correspond à celle que nous avons développée lors de divers travaux réalisés dans le sud de la France (Ducasse et Langlais 2007, Langlais 2007 et 2010, Ducasse 2010). 


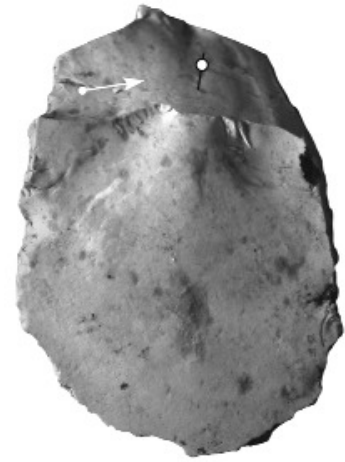

1
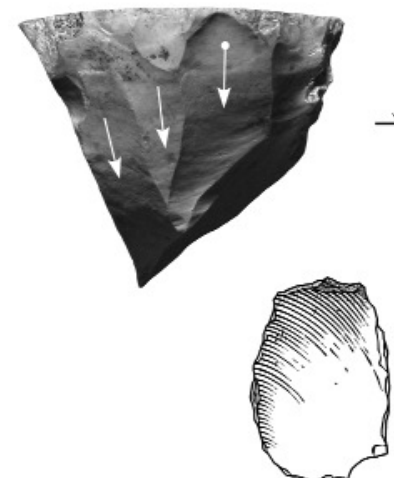

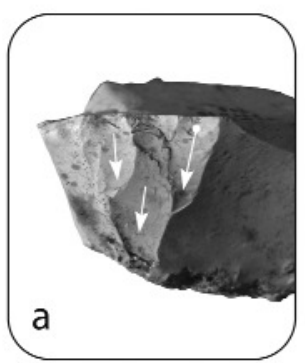

(1)

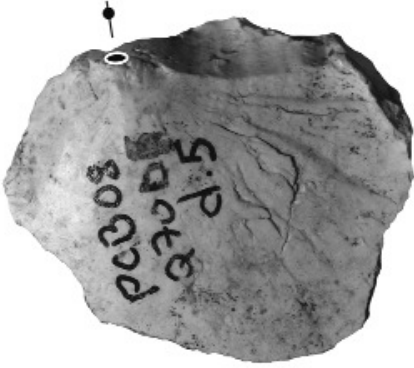

1
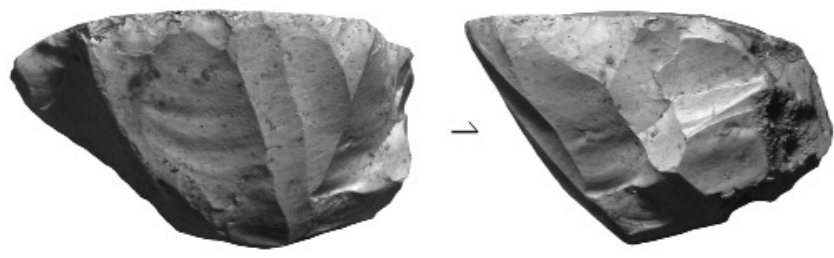

1

(3)

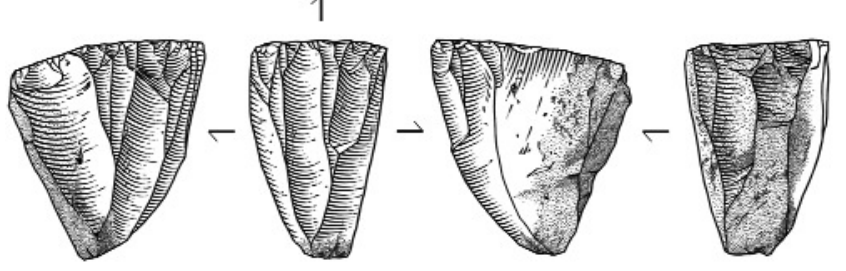

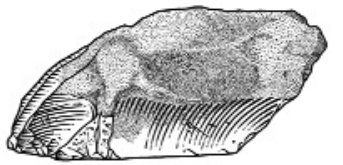

l

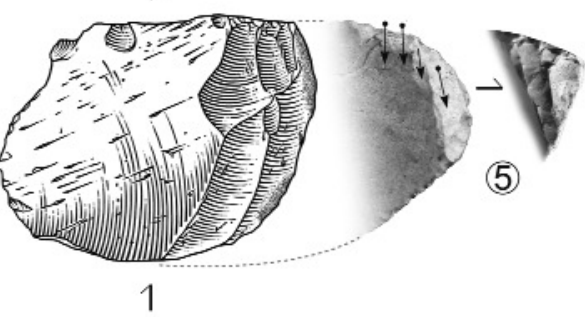

(4)
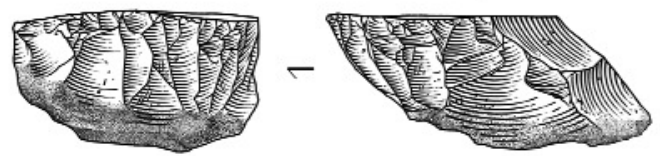

Figure 12 - Variabilité des modalités de production des supports de lamelles à dos dextre marginal ( $n^{\circ} 1$ et $4:$ nucléus " mixtes » ayant généré, sur des tables distinctes, des lamelles et des microlamelles, $n^{\circ} 3:$ nucléus pyramidal sur bloc (?), $n^{\circ} 4$ et $5:$ de la microlamelle vers la lamelle ?). Dessins et clichés S. Ducasse.

Figure 12 - Variability of the production types that are employed to obtain bladelet with right-hand and marginal back blanks $\left(n^{\circ} 1\right.$ and 4: mixed cores that permit to obtain, on distinctive flacked surfaces, bladelets and microbladelets, $n^{\circ} 3$ : " pyramidal »core on block (?), $n^{\circ} 4$ and 5: from microbladelets to bladelets?). Drawings and pictures S. Ducasse.

à partir de tables carénées, souvent convergentes qui, installées en face supérieure de l'éclat (caractère « dorsal »), sont exploitées à partir de leur face inférieure (ex. grattoir caréné : fig. $15, \mathrm{n}^{\circ} 1$ et $4, \mathrm{~N}=13$ ). Bien plus original, le débitage « sur front ventral déjeté » est quant à lui orienté de manière semi-transversale par rapport à l'axe d'allongement du support sélectionné, d'ailleurs fréquemment laminaire. Installée cette fois en face inférieure (caractère « ventral »), la surface débitée doit son « déjettement » à la nature du plan de frappe : il s'agit généralement d'un pan naturel de la face supérieure (fig. $15, n^{\circ} 5$ et 6 ). Ce type de nucléus, décrit pour la première fois à Thèmes (cf. nucléus «grattoir/burin »: Le Brun-Ricalens et Brou, 2003), a parfois été comparé aux "pièces de la Bertonne ${ }^{19}$ bien qu'il s'en distingue à notre sens nettement, comme en témoigne leur présence conjointe dans certaines séries (à la Bombetterie par exemple : Cheynier 1956, fig. 5, $\mathrm{n}^{\circ} 12$ et fig. 6, $\mathrm{n}^{\circ} 21$ ou Ducasse et Langlais 2008, fig. $12, n^{\circ} 6$ et 7 ). À ce titre, la réévaluation en cours de la collection lotoise dite de « la

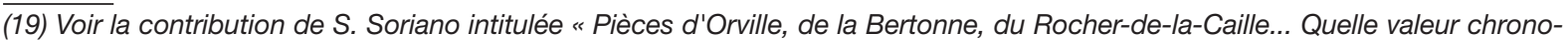
culturelle pour le Paléolithique supérieur ancien sur la marge sud-est du Bassin parisien ? » et disponible dans le rapport 2004 du PCR "Le Paléolithique supérieur ancien au centre et au sud du Bassin parisien. Des systèmes techniques aux comportements » codirigé par P. Bodu, L. Chehmana et N. Teyssandier. 
(1) Un support prédéterminé

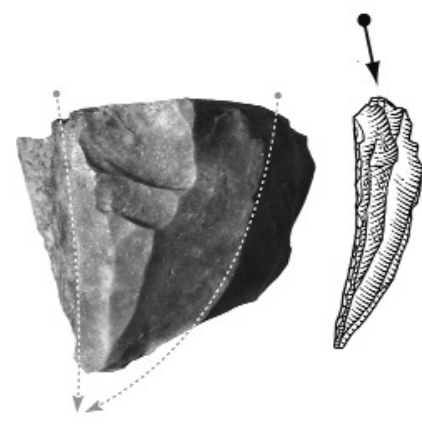

Table dissymétrique

Flanc droit parallèle

à l'axe d'allongement

de la surface de débitage

Flanc gauche

convergent vers la

base du volume

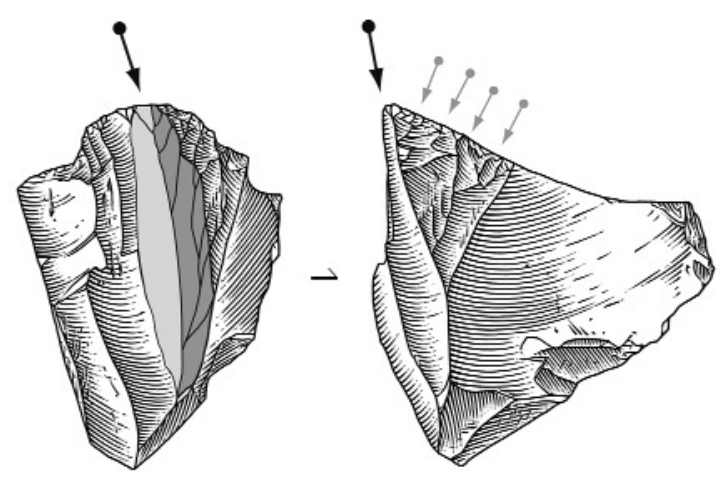

(2) Des modifications post-débitage accentuant la silhouette naturelle du support

pointe naturelle

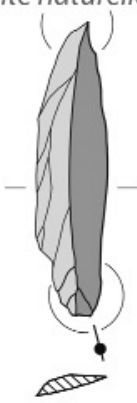

proximale

fréquente
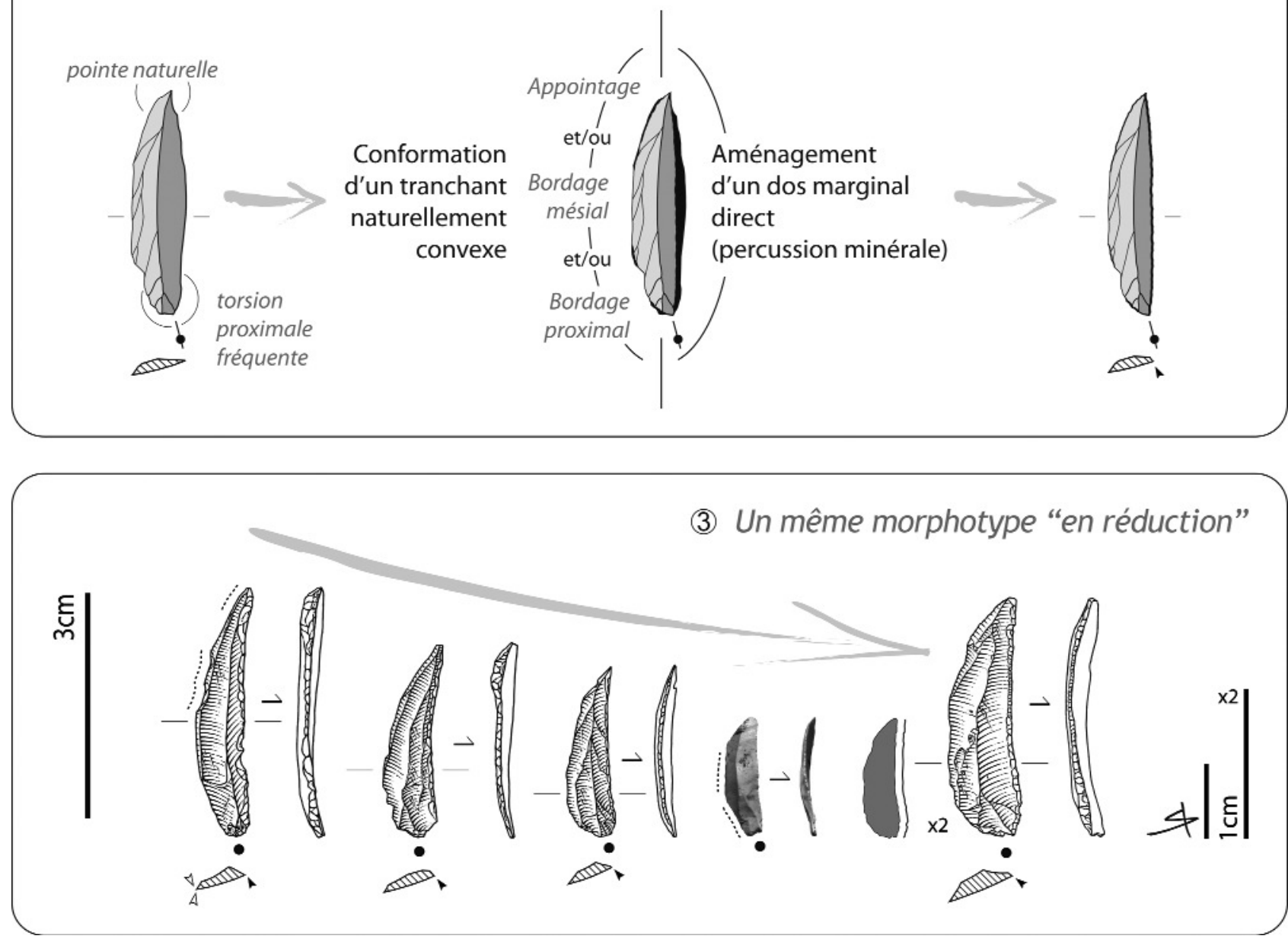

Figure 13 - De la production à la transformation des lamelles dextrogyres, une composante originale de la couche 4 du Petit Cloup Barrat. Dessins, clichés et infographie S. Ducasse.

Figure 13 - From the production to the transformation of the bladelet with right-hand and marginal back blanks: an original component of the layer 4 lithic industry. Drawings, pictures and computer graphics S. Ducasse. 

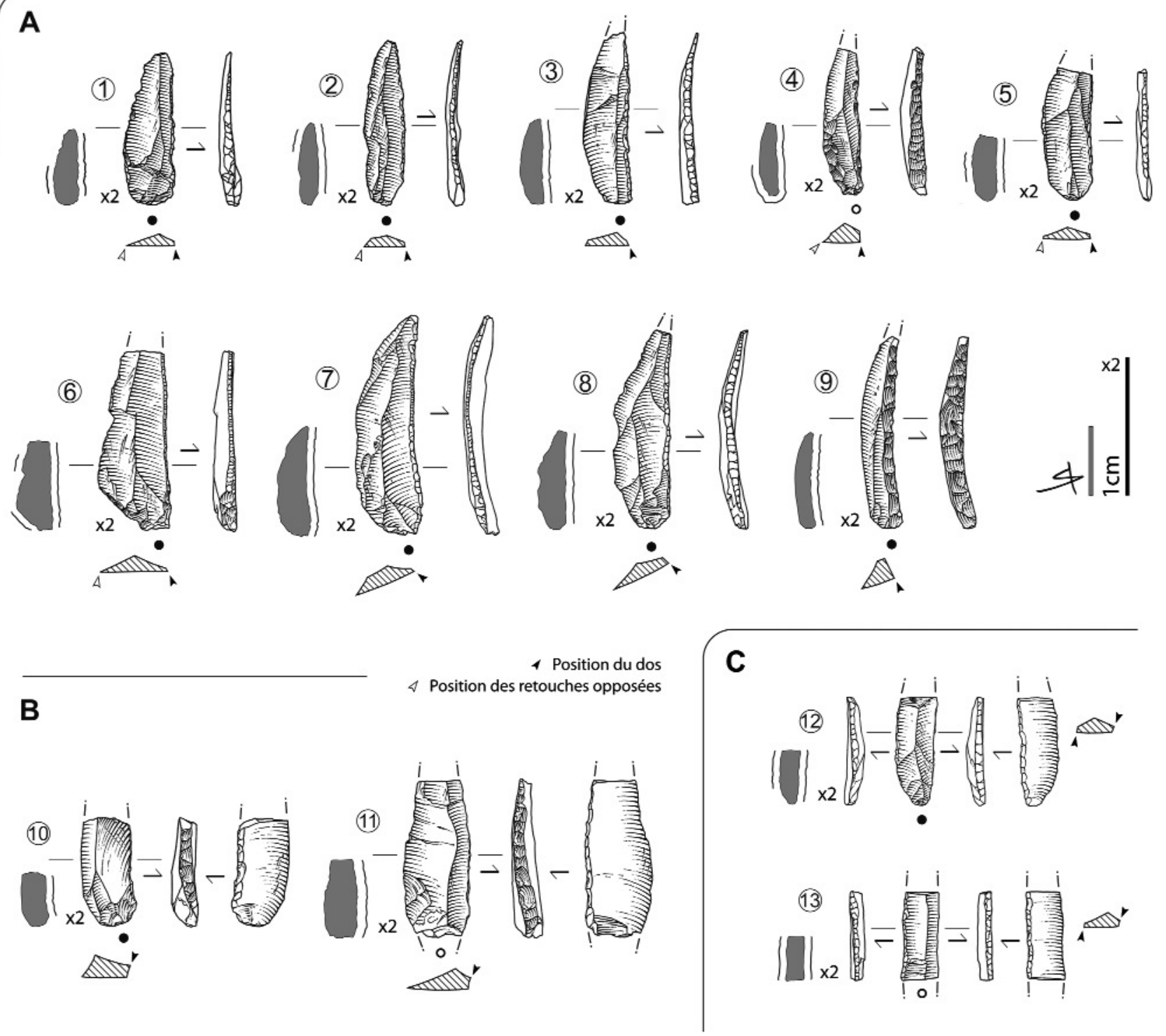

Figure 14 - Exemples de microlamelles à dos dextre direct $(A)$, inverse $(B)$ et alterne (C). Dessins S. Ducasse.

Figure 14 - Examples of backed microbladelets (A: direct retouch, B: inverse retouch, C: alternate retouch). Drawings S. Ducasse.

Cave à Endives » (Raux et Piel-Desruisseaux 1997), tout en nous conduisant à discuter de son origine (anciennes fouilles menées au Cloup Barrat ${ }^{20}$ ), illustre cette confusion fréquente : l'une des deux « pièces de la Bertonne » publiées (op. cit., fig. 4, n 14 et 15) correspond après examen à un nucléus "sur front ventral déjeté " typique (fig. $15, n^{\circ}$ 8) tandis que la seconde doit être classée dans la catégorie des nucléus microlamellaires " sur tranche transversale à encoche " (fig.15, $\mathrm{n}^{\circ} 7$ ), modalité également présente au sein de la couche 4 . L'existence du débitage « plan préférentiel sur tranche transversale " (Ducasse et Langlais 2008) au Petit Cloup Barrat n'est relayée que par quelques indices ténus, tous issus du fond de l'abri : outre la pièce illustrée (fig. $11 n^{\circ} 3$ ) liée semble-t-il à la recherche de supports de lamelles à dos dextre marginal (la « troncature » inverse partielle jouant a priori un rôle de recintrage), notons une possible microlamelle à pan « cannelé » en T72B (ou lamelle « type Orville » : Perlès 1982).

Avec 33 nucléus sur 79 (soit $41 \%$ du total), les productions dites « sur tranche » constituent la tendance la plus marquée de cet assemblage. Quelle que soit

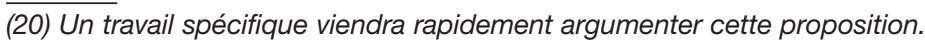




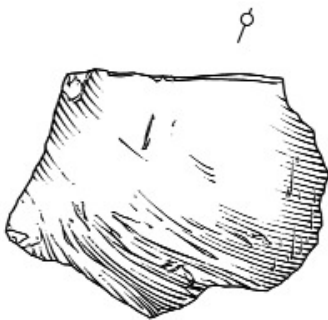

1

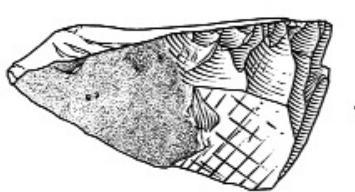

(1)

(2)
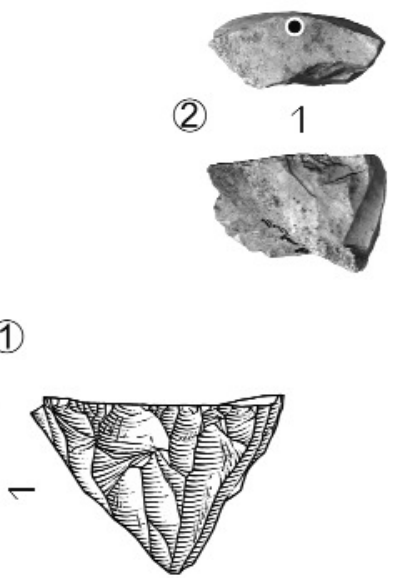

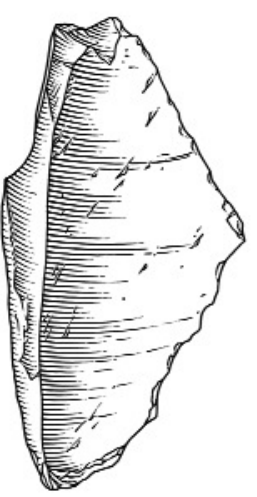

1
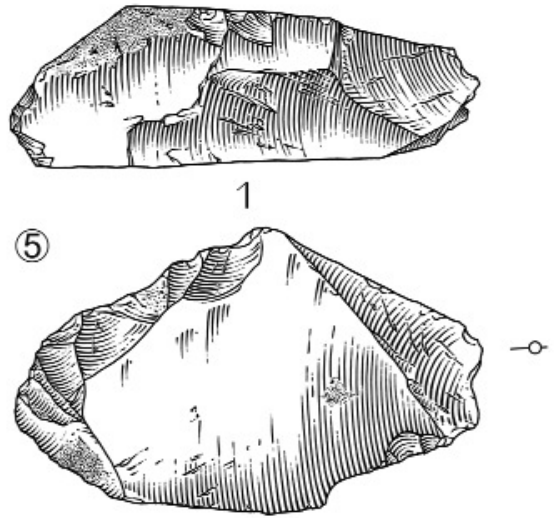

I
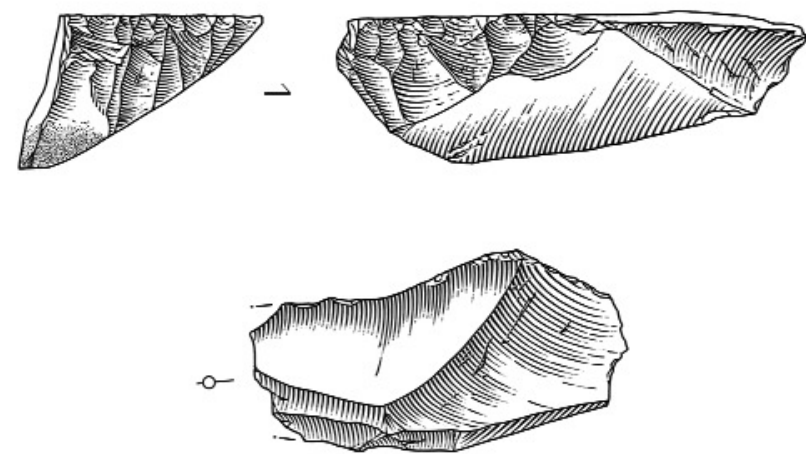

1
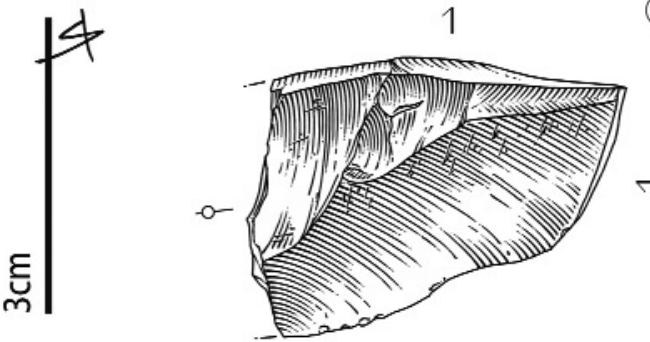

(6)

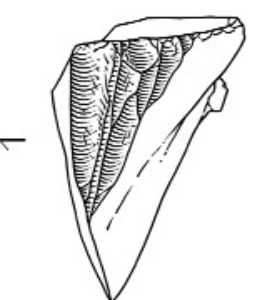

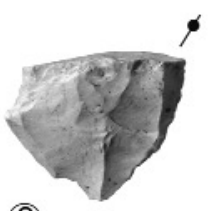

(3)

(4)

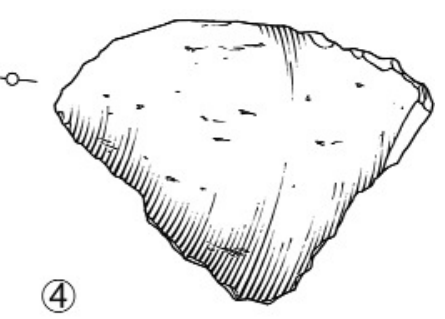

1
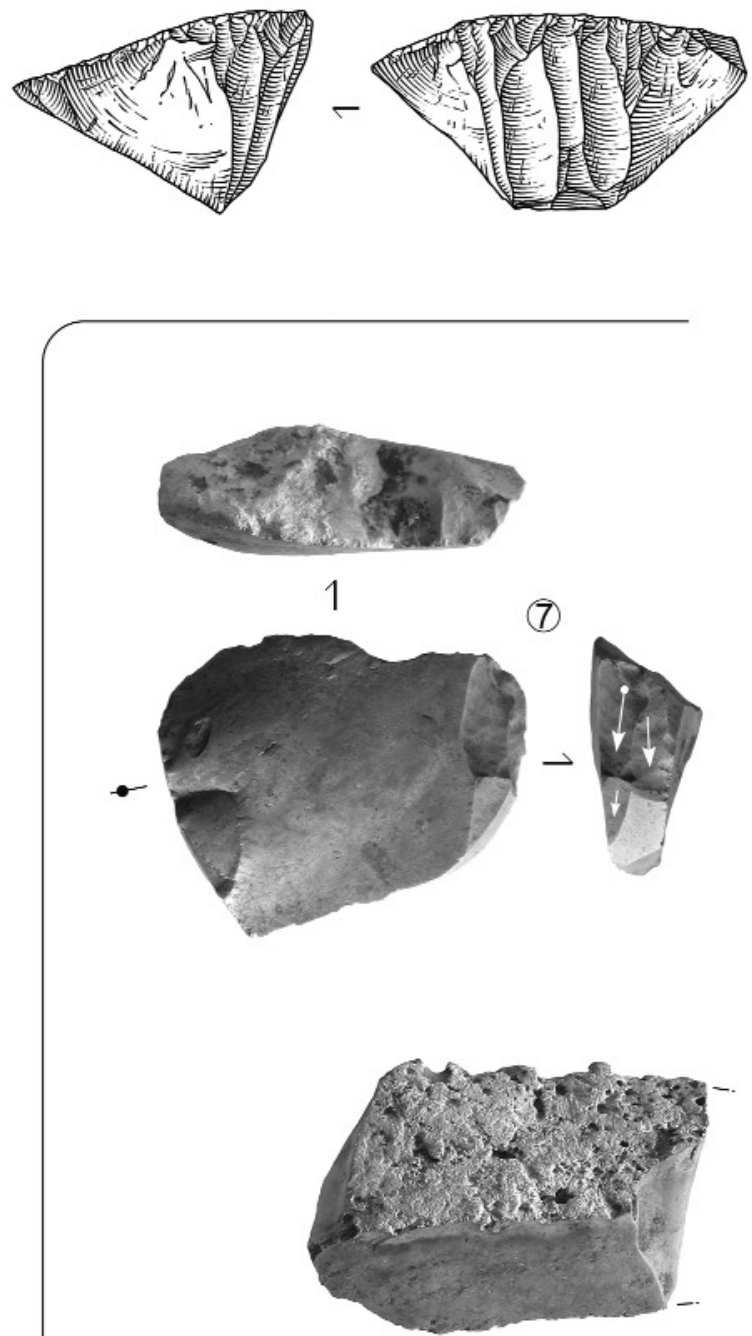

(8)

1
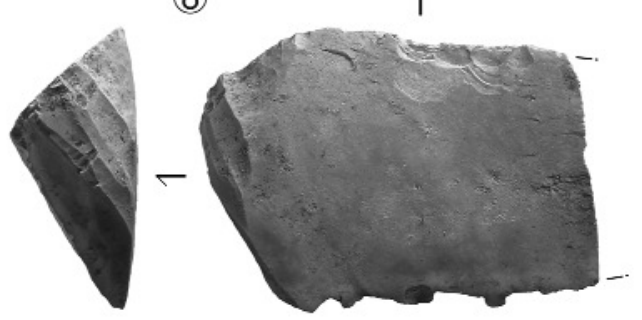

Figure 15 - Exemples de nucléus à microlamelles dits sur «front d'éclats », sous-produits associés ( $n^{\circ} 1$ et 4 : « sur front dorsal », $n^{\circ} 2$ : éclats fronto-latéral, $n^{\circ} 3$ : éclat latéral, $n^{\circ} 5$ et 6 : "sur front ventral déjeté ») et « fausses » pièces de la Bertonne provenant de la Cave à Endives ( $n^{\circ} 7$ : nucléus à microlamelles sur tranche transversale à encoche, $n^{\circ} 8$ : nucléus " sur front ventral déjeté » - cf. Raux et Piel-Desruisseaux 1997, fig. 4, $n^{\circ} 14$ et 15). Dessins et clichés S. Ducasse.

Figure 15 - Examples of microbladelets cores so-called « sur front d'éclat », by-products ( $n^{\circ} 1$ and 4: " sur front dorsal » core or scraper core-like, $n^{\circ} 2$ and 3: lamellar flakes, $n^{\circ} 5$ and 6: "sur front ventral déjeté » core) and false "Bertonne cores »from « la Cave à Endives » $\left(n^{\circ}\right.$ 7: microbladelets core "sur tranche transversale " with side notch, $n^{\circ}$ 8: " sur front ventral déjeté " core - cf. Raux et PielDesruisseaux 1997, fig. 4, $n^{\circ} 14$ et 15). Drawings and pictures S. Ducasse. 
(1)

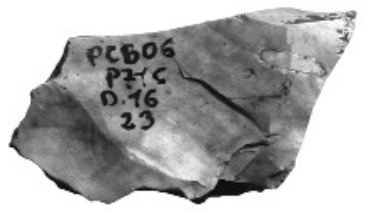

1
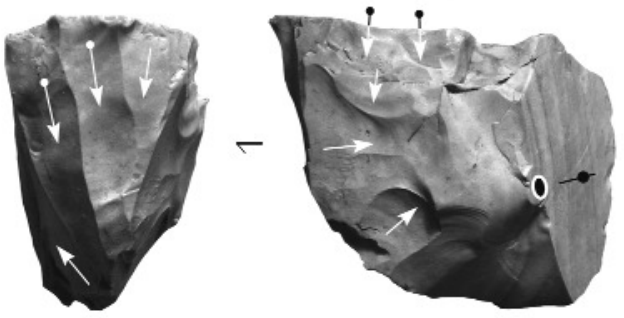

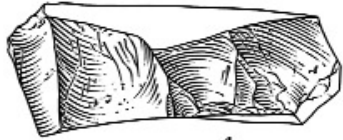

1

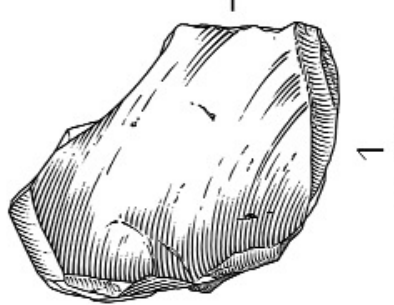

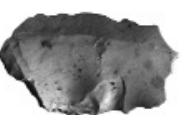

(3)
(2)

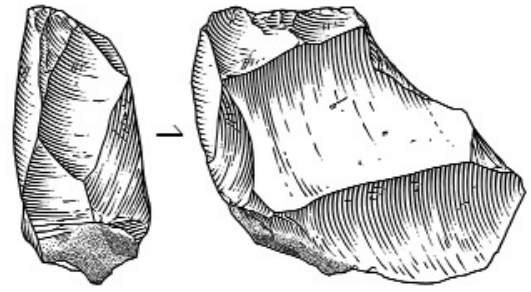

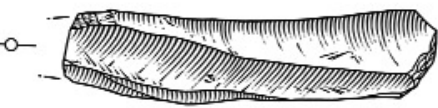

1

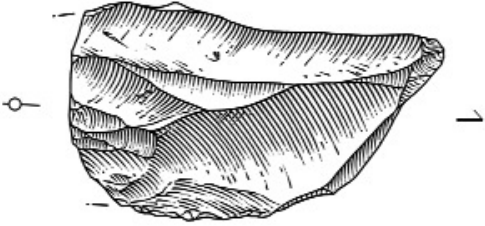

(4)
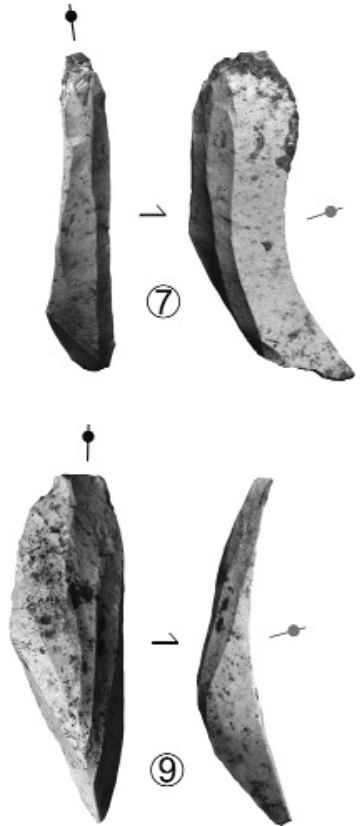

$\sim$
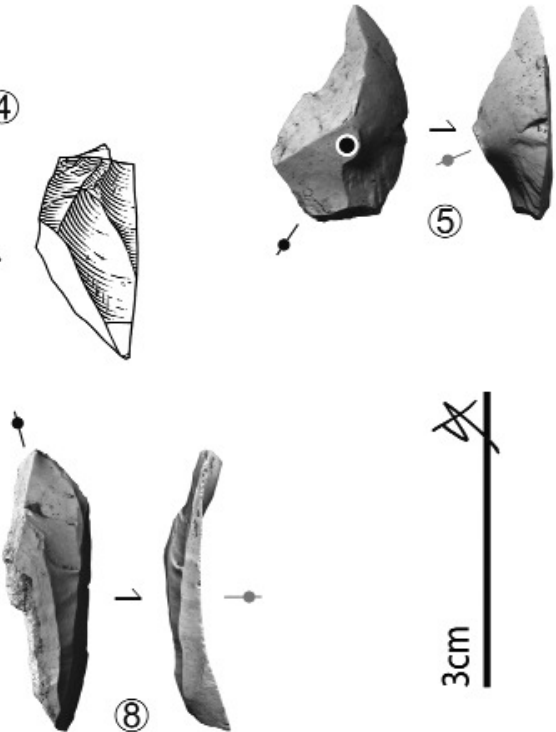

(11)
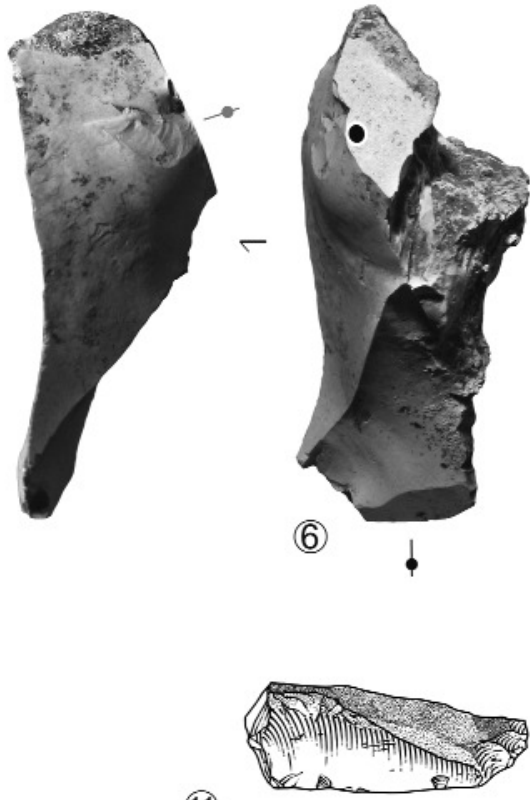

1
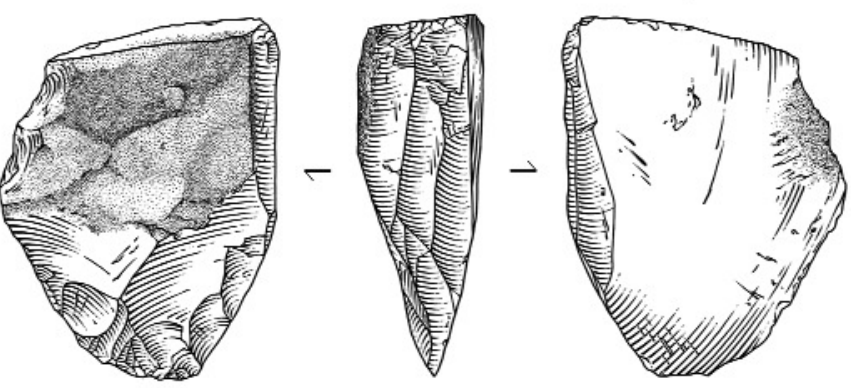

Figure 16 - Exemples de nucléus à lamelles et microlamelles dits sur " tranche d'éclats » et sous-produits associés ( $n^{\circ} 1:$ nucléus sur tranche transversale - recherche de supports convergents, $n^{\circ} 2$ : nucléus à microlamelles sur tranche transversale à encoche, $n^{\circ} 3$ : probable éclat de façonnage de plan de frappe correspondant, $n^{\circ} 4$ : nucléus à microlamelles sur tranche transversale, $n^{\circ} 5$ et $6:$ éclats d'ouverture de table ayant permis d'ôter la partie proximale des supports exploités, $n^{\circ} 7$ à 10 : éclats lamellaires sur tranche, $n^{\circ} 11$ : nucléus à lamelles sur tranche transversale). Dessins et clichés S. Ducasse.

Figure 16 - Examples of bladelets and microbladelets cores so-called « sur tranche d'éclat » and by-products ( $n^{\circ}$ 1: « sur tranche transversale » core - search for convergent blanks, $n^{\circ} 2$ : "sur tranche transversal » with side notch microbladelets core, $n^{\circ} 3:$ rejuvenation core flake (stricking plateform), $n^{\circ} 4$ : " sur tranche transversale » microbladelets core, $n^{\circ} 5$ and $6:$ products from the opening of flake surfaces, $n^{\circ} 7$ to 10: lamellar flakes, $n^{\circ} 1$ : "sur tranche transversal » bladelets core). Drawings and pictures $S$. Ducasse. 

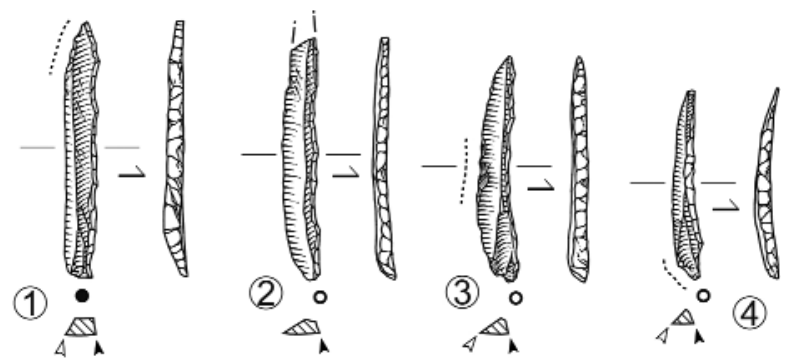

\section{Position du dos}

$\checkmark$ Position des retouches opposées

Figure 17 - Lamelles à dos dextre étroites. Dessins S. Ducasse.

Figure 17 - Narrow and right-hand backed bladelets. Drawings S. Ducasse.

l'orientation de la table sur le volume exploité (transversale, longitudinale) ou le type d'aménagement du plan de frappe observé en fin d'exploitation (le plus souvent lisse, parfois aménagé par un facettage latéral), cette modalité a été mise en œuvre afin de produire, selon les cas, des supports lamellaires et microlamellaires rectilignes (fig. 16, $\mathrm{n}^{\circ}$ 11) à tors comme nous l'avons vu précédemment (cf. infra). L'étroitesse de certaines tables conjuguée à l'abondance de lamelles rectilignes à pan revers brutes (munies ou non de néo-crêtes : tabl. 4) doit notamment mais pas uniquement - être mise en relation avec la présence de lamelles à dos dextre étroites (fig. 17 ; 3$4 \mathrm{~mm}$ de large pour 1-2 d'épaisseur).

\section{3 - Bilan}

Nous sommes loin d'avoir épuisé le potentiel informatif de l'industrie lithique de la couche 4 , actuellement riche de plus de 25000 pièces. Si de nombreux aspects restent à aborder plus finement (typologie des armatures, relation modalités/objectifs dans le cadre des productions lamellaires, etc.), rappelons qu'il s'agit d'un assemblage " ouvert » à propos duquel rien ne dit que la poursuite des fouilles n'amènera pas - parallèlement à une expansion quantitative du corpus - son lot de surprises.

En l'état, la gestion techno-économique des équipements lithiques de la couche 4 est construite autour de trois composantes dont certaines sont imbriquées. Alors que la confection de l'outillage de transformation est assurée par la mise en œuvre, différée ou non, d'un débitage laminaire auquel s'associe une production autonome et locale d'éclats, la fabrication (et/ou réfection) des armes de chasse passe le plus souvent par une ramification des chaînes opératoires : la production de lamelles et en effet liée à l'exploitation de « matrices » pré-débitées (éclats épais, allongés, lames) produites intentionnellement ou récupérés parmi les sous-produits laminaires. Ces productions, aux objectifs et modalités très diversifiés comme nous l'avons vu, alimentent donc un corpus d'armatures lamellaires tout aussi varié, au sein duquel les lamelles (et microlamelles) à dos dextre marginal se singularisent, constituant un morphotype original sur lequel nous reviendrons.

\section{4 - Caractérisation des vestiges osseux : l'alimentaire (JCC)}

Le gisement du Cloup Barrat est particulièrement riche en vestiges fauniques. La base de données, intégrant les pièces enregistrées lors de la fouille ainsi que les éléments extraits du tamisage car considérés comme déterminables, comporte 3647 restes attribuables à la couche 4 . Leur conservation est variable, allant d'un très bon aspect des surfaces osseuses dans la partie abritée où se développent les ensembles attribués au Solutréen et au Badegoulien, à une conservation plus médiocre à l'extérieur.

Le matériel osseux de la couche 4 du Cloup Barrat revêt un intérêt particulier dans la mesure où il s'agit d'un des rares assemblages contemporains du DMG dont la fouille récente permet d'y développer pleinement les techniques de l'archéozoologie. Avec Gandil (Griggo 1997) c'est même le seul pour l'Aquitaine orientale. Même si la surface fouillée est encore assez restreinte, sa caractérisation apporte des éléments de compréhension des comportements humains à l'aube du Magdalénien " classique ».

\section{1 - Analyse dimensionnelle des fragments par type de tissu osseux}

La procédure d'analyse des restes osseux mise en œuvre est classique : la première étape concerne la totalité des fragments osseux avant détermination ; un décompte est réalisé par classe centimétrique, par type de tissu osseux (compact, spongieux, côtes, dents, bois de cervidés) et intègre la combustion. Compte tenu de l'homogénéité de nombreux paramètres (taphonomiques, choix des taxons et modalités d'exploitation), l'analyse porte sur un échantillon représentatif correspondant à la moitié de la surface attribuée à la couche 4 ; les vestiges ainsi décomptés sont au nombre de 60790. Nous ne détaillerons pas cette analyse fastidieuse ici mais il peut être précisé que les variations latérales ou verticales des catégories descriptives retenues sont faibles.

L'examen de la distribution par classe dimensionnelle (fig. 18A) et par type de tissu osseux présent (fig. 18B) indique la présence d'une grande quantité de petits os parmi lesquels une forte proportion d'os brûlés. Les os compacts sont beaucoup plus abondants que les os spongieux que ce soit parmi les os brûlés ou les os non-brûlés.

Les os enregistrés dans la base de données archéozoologique correspondent à ceux qui ont été enregistrés en trois dimensions dès $25 \mathrm{~mm}$, accompagnés des vestiges plus petits mais déterminables. La longueur des vestiges est relativement réduite (fig. 19) : rares sont les fragments dépassant $70 \mathrm{~mm}$ de longueur. Parmi les 32 fragments de plus de $100 \mathrm{~mm}$, on trouve sept fragments de bois de renne, deux côtes, une extrémité articulaire; le reste correspond à des diaphyses. 


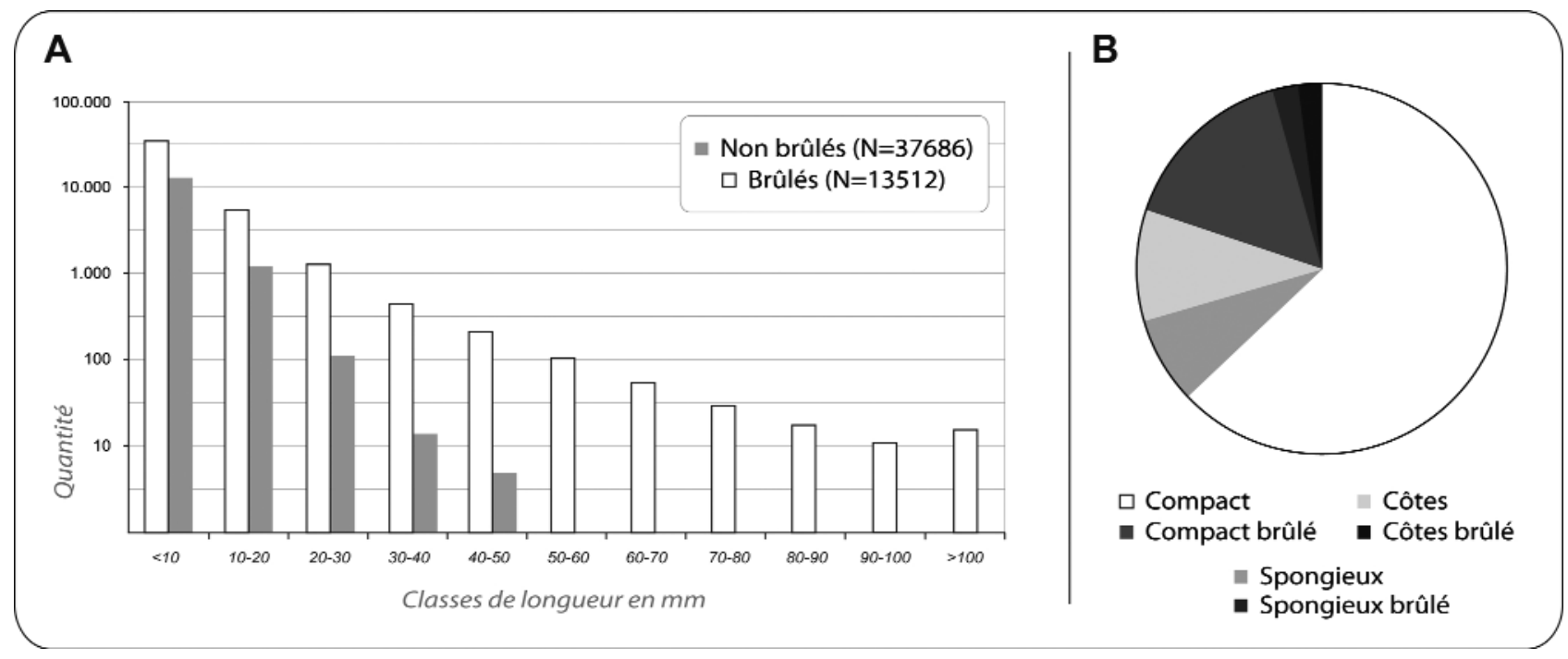

Figure 18 - $A$ - Longueur des vestiges osseux récoltés pour un échantillon couvrant la moitié de la surface fouillée de la couche 4 (NR = 60790, échelle logarithmique). B - Proportions des différents types de tissus osseux observés dans la classe dimensionnelle de 10 à $40 \mathrm{~mm}(\mathrm{NR}=1600)$.

Figure 18 - A - Length of Faunal remains for a sample covering the half of the level 4 excavation (NISP=60790, logary scale). $B$ - Proportions of bone tissues for each centimetric class from 10 to $40 \mathrm{~mm}(\mathrm{NISP}=1600)$.

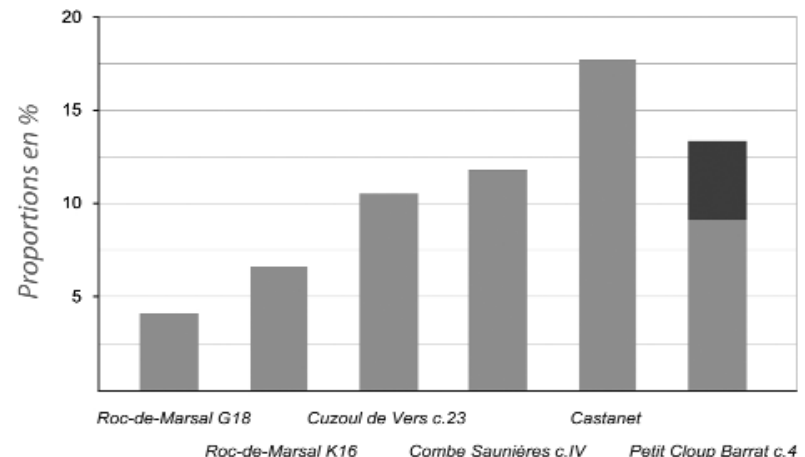

Figure 19 - Proportions des fragments de longueur supérieure à $80 \mathrm{~mm}$ parmi les os récoltés de plus de $40 \mathrm{~mm}$ au sein de quelques gisements paléolithiques du sud-ouest de la France à renne dominant. Pour la couche 4 du Cloup Barrat sont figurées en gris clair les valeurs pour l'extérieur et en gris foncé les valeurs pour le fond de l'abri.

Figure 19 - Proportion of bone fragments with length above 80 $\mathrm{mm}$ among those more $40 \mathrm{~mm}$ in some Upper Paleolithic sites in south-west France with reindeer as main species. For the level 4 from the Cloup Barrat comparison is made between the outside part in gray, and inside of the shelter in dark grey.

\section{2 - Etat de surface des restes déterminés}

L'examen de la conservation des vestiges organiques a concerné un échantillon uniformément réparti de 678 restes dont 426 restes osseux de plus de $30 \mathrm{~mm}$ - soit environ $24 \%$ de l'assemblage total de la couche 4 . Les surfaces osseuses ne sont pas très bien conservées (fig. 20). L'érosion et l'émoussé des angles concernent plus des trois quarts des vestiges (tabl. 6). Les empreintes de racines sont également présentes. Dans l'ensemble, ces vestiges sont peu favorables à l'examen des marques d'origine anthropiques.

Des 48 dents examinées dans cet échantillon, seules deux sont parfaitement conservées ; les autres sont plus ou moins dégradées ce qui rend l'assemblage peu informatif pour préciser les saisons de chasses et l'âge des proies. Par comparaison, la couche $8 \mathrm{c}$ identifiée dans la grotte est bien mieux conservée (Castel et al. 2005 et 2006).

II faut, en outre, remarquer que l'état de conservation se dégrade plus on s'éloigne de la partie abritée, le matériel du carré S70 étant mieux conservé que celui de P70. Curieusement, le matériel localisé vers le nord-est est un peu mieux préservé que celui du quart sud-est.

\section{3 - Les espèces identifiées}

Une large majorité des vestiges de la base de données a été déterminée anatomiquement ou taxonomiquement. Les déterminations à la fois anatomiques et taxonomiques concernent une partie de l'assemblage plus limitée. Le renne domine largement devant le bouquetin et le cheval (tabl. 7).

L'importance relative du renne et du bouquetin dans la composition faunique est délicate à préciser puisque la fracturation et la fragmentation des os ne permettent pas d'attribuer de manière spécifique des fragments déterminés anatomiquement mais de petites dimensions (fig. 21A). Si l'on considère que les carnivores de taille moyenne (l'hyène principalement ainsi que le loup et l'ours pour certaines portions de leurs squelettes) sont absents 


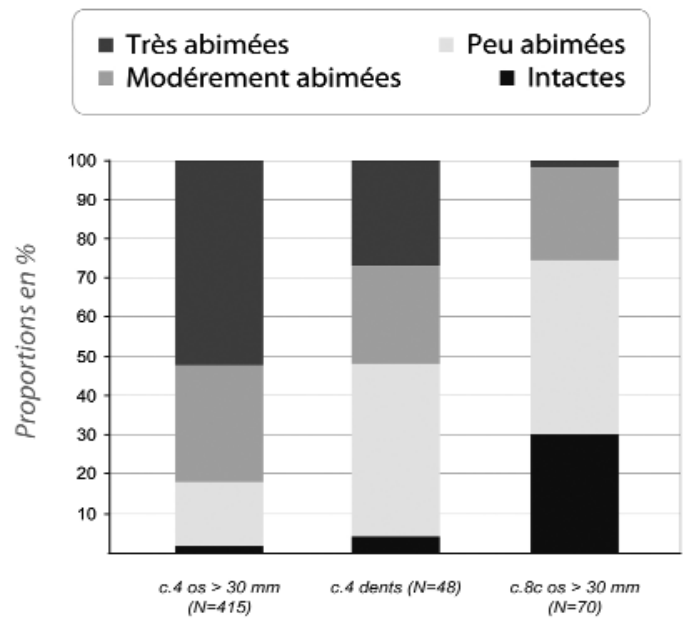

Figure 20 - Conservation des surfaces osseuses ; comparaison des couches 4 et $8 \mathrm{c}$.

Figure 20 - Preservation of bones surfaces. Comparison of levels 4 and $8 \mathrm{c}$.

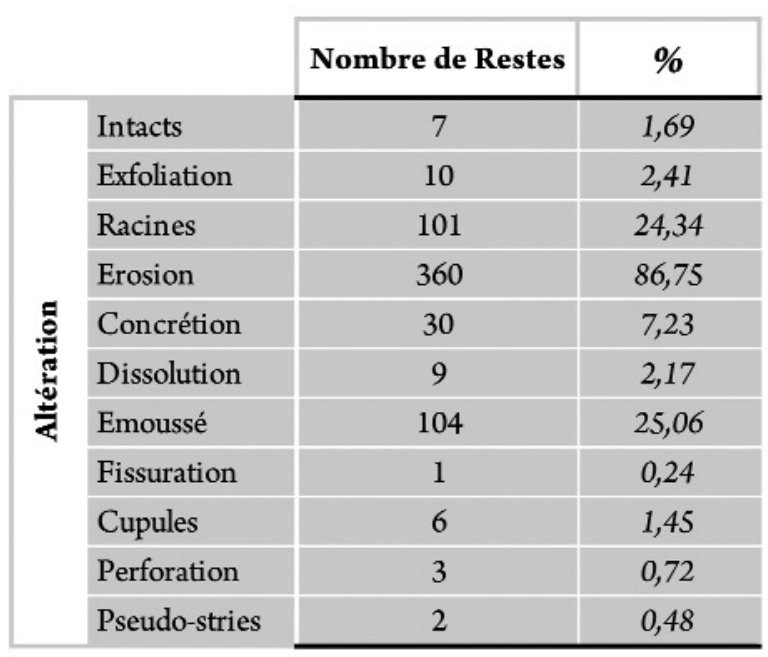

Tableau 6 - Occurrences des modifications superficielles sur un échantillon de 415 os de plus de $30 \mathrm{~mm}$ de longueur. Sept sont intacts, les autres portent au moins une sorte d'altération.

Table 6 - Superficial modifications observed on a sample of 415 bones that are more than $30 \mathrm{~mm}$ long. Seven are intact; other bare at least one modification.

de l'assemblage, on peut alors accepter l'hypothèse que tous les restes attribués à la classe des mammifères de taille moyenne correspondent au renne ou au bouquetin. Si 797 vestiges sont incontestablement du renne et $45 \mathrm{du}$ bouquetin, 2272 peuvent correspondre à l'une ou l'autre espèce et 112 peuvent correspondre au renne, au bouquetin ou au chamois.

Trente-neuf des 45 restes de bouquetin sont des dents ; à la différence de certaines couches du Cuzoul de Vers

\begin{tabular}{|l|c|}
\cline { 2 - 2 } & Nombre de Restes \\
\hline Grands mammifères indéterminés & 77 \\
\hline Moyens ou grands mammifères indéterminés & 106 \\
\hline Mammifères de taille moyenne indéterminés & 1741 \\
\hline Renne ou bouquetin & 425 \\
\hline Bouquetin ou chamois & 4 \\
\hline Petits ou moyens mammifères indéterminés & 87 \\
\hline Petits ou moyens ongulés indéterminés & 21 \\
\hline Petits mammifères indéterminés & 3 \\
\hline Chamois ou saïga & 8 \\
\hline Très petits vertébrés & 8 \\
\hline Indéterminés & 242 \\
\hline & \\
\hline \multicolumn{1}{|c|}{ Total } & $\mathbf{2 7 2 2}$ \\
\hline
\end{tabular}

Tableau 7 - Représentation des différents taxons identifiés (classement par classe de taille).

Table 7 - Number of remains of identified species (ranked by body size).

(Castel 1999, 2010) seule une petite partie sont des incisives (NR=9). Par conséquent, il n'y a pas d'acquisition spécifique d'incisives ; la présence de dents jugales et de quelques restes postcrâniens permet de suspecter que des éléments du squelette de plusieurs individus peuvent être présents parmi les vestiges attribués sans plus de précision aux ongulés de taille moyenne. Dans ces conditions, les parties du squelette qui permettent la meilleure distinction du renne et du bouquetin sont les os du bas des pattes. Ces os attribués au bouquetin étant rares dans la couche 4 , il faut donc admettre que le squelette de ce taxon est bien moins abondant que celui du renne. Toutefois, cette imprécision relative nous conduit à examiner ces deux taxons conjointement pour les analyses de représentation anatomique, de distribution des stries, ou des modalités de combustion.

\section{4 - Existe-t-il un choix des espèces chassées?}

L'une des principales phases d'accumulation de l'Igue du Gral, piège naturel localisé de l'autre coté de la vallée du Célé à $4 \mathrm{~km}$ (fouille en cours, direction J.-C. Castel), s'insère dans la deuxième partie du DMG. Cinq dates sont en effet disponibles entre 14500 et 17000 BP (Castel et al. 2008 ; Castel et Chauvière à paraître). Elles correspondent à des ensembles paléontologiques dans lesquels renne, cheval et bison sont invariablement associés et dominants au cours des différentes saisons de fréquentation du Causse. L'assemblage de la couche 4 avec ses rennes et ses bouquetins, qu'accompagne un peu de cheval, semble donc montrer une chasse préférentielle centrée sur des ongulés de taille modeste alors que l'environnement proche du site permettait d'en chasser de plus gros. Seule une analyse très détaillée des saisons de chasse ou de chute dans l'aven permettrait de l'affirmer avec certitude (travaux en cours). 
A

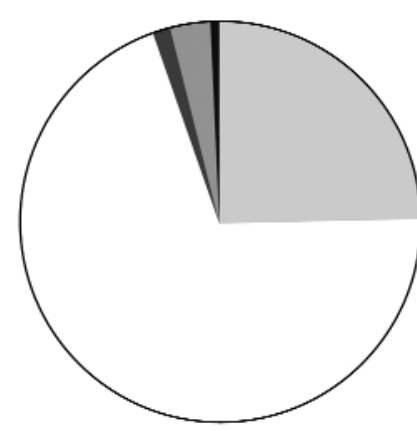

Renne $\square$ Renne ou bouquetin

- Bouquetin

- Chamois

- Petit ou moyen mammifère indét.
B

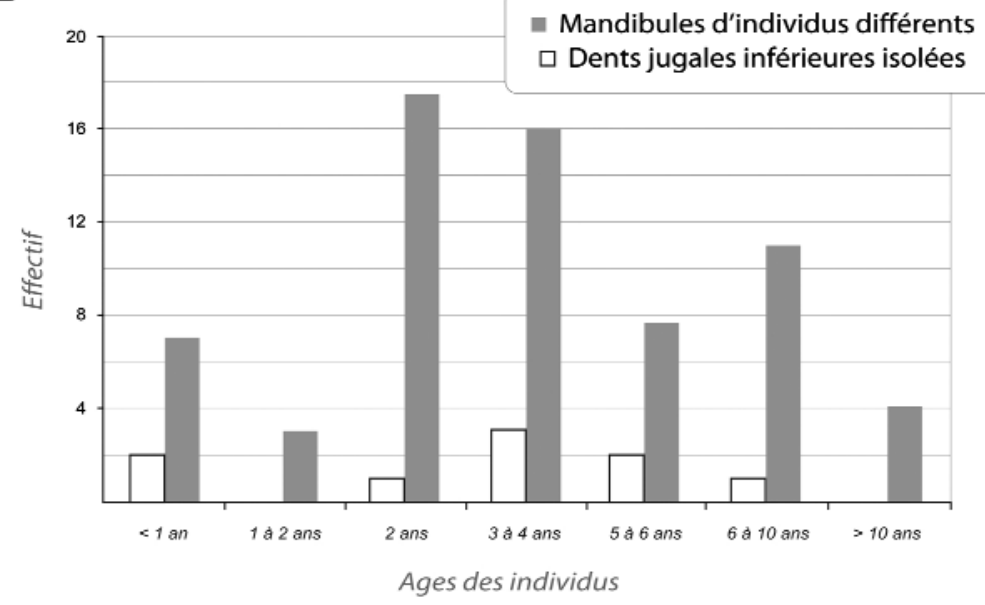

Figure 21 - $A$ - Proportions de renne, de bouquetin et de chamois. B - Age des rennes d'après l'éruption et l'usure dentaire (évaluation préliminaire à partir des tables de Bouchud).

Figure 21 - A- Proportions of reindeer, ibex and chamois. B - Age of reindeer according to teeth eruption (preliminary evaluation according to Bouchud 1966).

\section{5 - Age des proies et saisons d'occupation}

Les vestiges dentaires sont relativement abondants mais peu de dents sont encore sur les mâchoires. Les données que nous présentons sont préliminaires avec une conversion de l'usure observée en âge telle que présentée par Bouchud (1966). Cette méthode est facile à mettre en œuvre et autorise une fiabilité suffisante dans le contexte particulier de la couche 4 . Toutes les classes d'âges sont représentées avec une légère surreprésentation des jeunes adultes (fig. 21B). Le sous-effectif des plus jeunes peut être le résultat de la conservation assez moyenne des vestiges (fragmentation différentielle des dents).

Les dents jugales déciduales sont au nombre de 19 dont 12 sont suffisamment complètes. Celles qui donnent des indications de saisons correspondent d'une part à un individu de deux mois ; sept dents signant d'autre part la présence d'au moins deux individus indiquant un âge de cinq à huit mois. L'été et la fin de la bonne saison sont représentés ; les autres saisons ne semblent pas l'être.

\section{6 - Représentation anatomique}

Comme indiqué, les diaphyses sont les parties les plus représentées (tabl. 8) en nombre de restes identifiés mais aussi en NMI (tabl. 9). La fréquence des parties squelettiques dépend fortement de la résistance des vestiges aux différentes formes d'érosion et de fossilisation. Sans entrer dans un calcul précis de la représentation en fonction de la densité osseuse, il ressort que les os longs sont représentés de façon relativement homogène. Le squelette axial postcrânien est bien plus rare. Une destruction post-dépositionnelle de ces os fragiles est évidente, mais il n'est pas exclu qu'une partie du squelette axial n'ait pas été introduite dans l'habitat. À noter, toutefois, l'abondance des côtes par rapport aux vertèbres que nous n'interprétons pas à ce stade des recherches.

\section{7 - Exploitation alimentaire et technique des carcasses}

Une quarantaine d'os seulement présente des stries de découpe. La quasi-totalité est localisée sur les diaphyses. Trois cependant correspondent à des stries de désarticulation. Des impacts de fracturation des os longs ont été identifiés mais ce type de stigmates est souvent difficile à diagnostiquer avec certitude. Les bords de fractures des os longs sont très fréquemment obliques avec des surfaces lisses ce qui atteste d'une fracturation systématique des diaphyses. De plus, la standardisation des longueurs des diaphyses est tout à fait similaire à ce qui peut s'observer dans des ensembles fauniques du Paléolithique supérieur mieux conservés (fig. 19). Aucun os long n'est complet et aucun ne dépasse le tiers de la longueur totale de l'os.

Les connexions et rapprochements anatomiques ont été recherchés systématiquement. Six ensembles ont été isolés pour l'instant. Le premier concerne deux dents jugales supérieures de cheval trouvées à quelques centimètres l'une de l'autre ; cela ne témoigne guère que de la disparition de l'os maxillaire correspondant. Les cinq autres remontages concernent des tarses de rennes (fig. 22) dont les os sont retrouvés à faible distance (les remontages réalisés sur des os éloignés n'ont pas été retenus pour des raisons de fiabilité). Ils indiquent 


\begin{tabular}{|l|c|}
\cline { 2 - 2 } & Nombre de Restes \\
\hline Crâne & 31 \\
\hline Dents & 308 \\
\hline Mandibule & 65 \\
\hline Hyoïde & 3 \\
\hline Vertèbres & 28 \\
\hline Côtes & 142 \\
\hline Cartilages ossifiés & 3 \\
\hline Sternèbres & 3 \\
\hline Ceintures & 20 \\
\hline Extrémités os longs & 123 \\
\hline Diaphyses & 2218 \\
\hline Patella & 2 \\
\hline Basipode & 52 \\
\hline Phalanges & 58 \\
\hline Phalanges vestigielles & 16 \\
\hline Sésamoïdes & 32 \\
\hline \hline & $\mathbf{3 1 0 4}$ \\
\hline \multicolumn{1}{|c|}{ Total } & \\
\hline
\end{tabular}

Tableau 8 - Fréquence des parties anatomiques toutes espèces confondues.

Table 8 - Frequencies of anatomical parts for all species together.

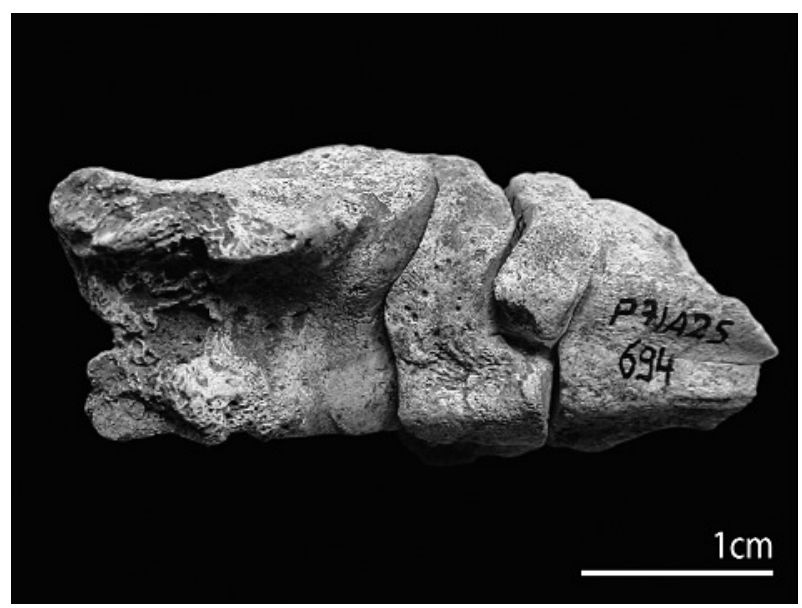

Figure 22 - Remontage sur membre postérieur de renne (du talus à l'extrémité proximale du métatarsien). Cliché J.-C.Castel.

Figure 22 - Refitting of posterior limb of reindeer (from talus to the metatarsal). Picture J.-C. Castel.

\begin{tabular}{|l|c|l|l|l|}
\hline & NME & droit & gauche & NMI \\
\hline Rocher & 14 & & & 7 \\
\hline Mandibule-condyle & 7 & & & 4 \\
\hline Axis & 2 & & & 2 \\
\hline Autres vertèbres & & & & 1 \\
\hline Côtes & & & & 1 \\
\hline Humérus-distum & & 6 & & 6 \\
\hline Humérus-foramen & 13 & & & 7 \\
\hline Radio-ulnaire-foramen & 7 & & & 4 \\
\hline Pyramidal & 6 & & 4 & 4 \\
\hline Métacarpien LFA & $520 \mathrm{~mm}$ & & & 7 \\
\hline Fémur-foramen & 14 & & & 10 \\
\hline Tibia-foramen & 19 & & & 4 \\
\hline Talus & 7 & 4 & & 5 \\
\hline Cubo-naviculaire & 8 & 5 & & 4 \\
\hline Grand cunéiforme & 8 & & & 4 \\
\hline Métatarsien LFA & $1425 \mathrm{~mm}$ & & & 1 \\
\hline le phalange & 38 & & & 1 \\
\hline 2e phalange & 14 & & & \\
\hline 3e phalange & 3 & & & \\
\hline & & & \\
\hline
\end{tabular}

Tableau 9 - Renne et bouquetin ; évaluation du MNI pour quelques parties du squelette représentatives (dents exclues). LFA : longueur cumulée des fragments de sillon antérieur.

Table 9 - Reindeer and ibex; evaluation of MNI of representative skeletal parts (teeth excepted). LFA: Total length of anterior groove from metapodial fragments. 
l'abandon sur place de tarses en connexion avec les extrémités des os longs correspondants (extrémité distale du tibia et extrémité proximale du métatarsien) après fracturation des diaphyses pour en retirer la moelle. En outre, la proximité des vestiges indique de faibles déplacements post-dépositionnels des parties squelettiques abandonnées. Après prélèvement de la viande, les potentialités alimentaires des os membres semblent donc avoir été exploitées par une fracturation autour des articulations permettant à la fois d'accéder à la moelle et d'isoler les articulations (au sens de Castel 1999, 2003,2010 ) et non par une désarticulation classique (au sens de Binford 1981).

Une proportion importante de la matière osseuse conservée est brûlée (fig.18A). Ces vestiges sont principalement de couleur noire ce qui implique une combustion intentionnelle et systématique. Les os de couleur marron, correspondant à une combustion incomplète, constituent une proportion non-négligeable (fig. 23). Cela résulte de la dégradation préférentielle des os de couleur grise ou blanche dans le contexte très érosif de la couche 4 et non de modalités de combustion particulières favorisant la forte représentation de vestiges peu brûlés. Une proportion importante de ces vestiges est de nature compacte que ce soit parmi les déterminés (fig. 23) ou les indéterminés (fig. 18B).

Au final, si la procédure d'exploitation des potentialités alimentaires et techniques des os des membres ressemble étroitement à celle décrite pour le Badegoulien du Cuzoul de Vers (Castel, 1999, 2003, 2010) elle n'est pourtant pas confortée par la présence d'une majorité écrasante d'articulation brûlées. L'identification d'articulations exclusivement non brûlées ne permet toutefois pas d'écarter que cette procédure de récupération de la graisse osseuse ait pu exister au sein de la couche $4 \mathrm{du}$ Cloup Barrat. La fonte post-dépositionnelle de l'os spongieux est fort possible et masque en effet tout critère d'appréciation.

\section{5 - Outillage, armement et parure sur matieres dures d'origine animale (F.-X. C)}

Parallèlement à l'utilisation de l'os comme combustible, l'exploitation technique des matières dures d'origine animale dans la couche 4 du Petit Cloup Barrat est aussi documentée par 198 pièces sur bois de renne, os, dent et coquille (tabl. 10). Leur transformation est orientée vers la réalisation de l'équipement matériel (outils et armes) et la confection de la parure corporelle. Le corpus est constitué des objets découverts à la fouille, à la suite du tri des refus de tamis et de l'ensemble des restes fauniques, selon des procédures désormais classiques (Chauvière 2003).

\section{1 - Le travail des bois de cervidé}

\subsection{1 - Présentation}

Les bois de cervidé de la couche 4 totalisent 168 vestiges inventoriés (tabl.11). Après raccords, le corpus s'élève à 166 pièces auxquelles s'ajoutent de nombreux fragments inférieurs à $1 \mathrm{~cm}$ non comptabilisés ici. L'état de conservation des volumes est moyen (émoussé des arêtes, traces de dissolution). En outre, la question de l'origine de la fragmentation (naturelle ou anthropique) de

\begin{tabular}{l|c|}
\cline { 2 - 2 } & Nombre \\
\hline Bois de cervidés & 166 \\
\hline Os & 10 \\
\hline Dent & 19 \\
\hline Coquille & 3 \\
\hline \hline \multicolumn{1}{|c}{ Total } & $\mathbf{1 9 8}$ \\
\hline
\end{tabular}

Tableau 10 - Matières dures d'origine animale travaillées (y compris fragments de bois de renne sans trace a priori).

Table 10 - Osseous industry (with fragments maybe unworked).
A

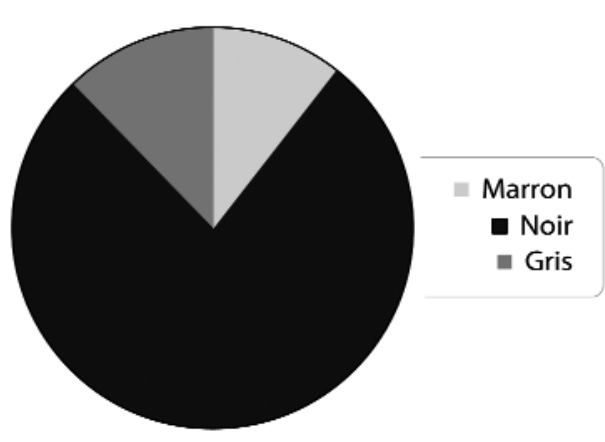

B

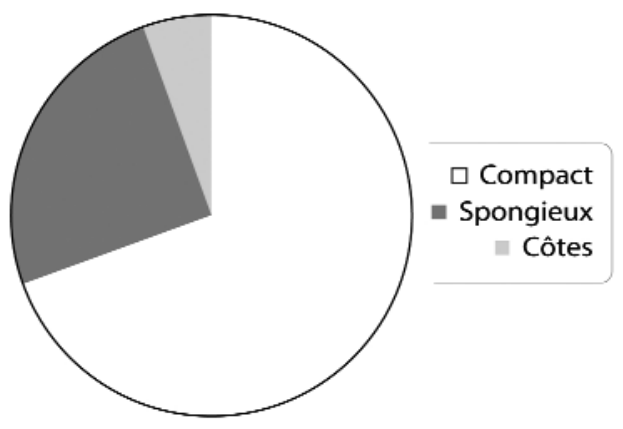

$N R=114 /$ Longueur moyenne $: 22,4 \mathrm{~mm}$

Figure 23 - Caractérisation des restes brûlés déterminés.

Figure 23 - Caracteristics of identified burned bones. 


\begin{tabular}{|c|c|c|}
\hline & Nombre & $\%$ \\
\hline Déchets du débitage & 42 & \multirow{5}{*}{25,30} \\
\hline dont base & (9) & \\
\hline dont cornillon & (1) & \\
\hline dont baguette brute & (5) & \\
\hline dont éclat & (27) & \\
\hline Déchets du façonnage & 4 & \multirow{3}{*}{2,41} \\
\hline dont baguettes régularisées & (2) & \\
\hline dont déchet de résection & (2) & \\
\hline Objets finis & 16 & \multirow{5}{*}{9,64} \\
\hline dont pointe (extrémité) & (5) & \\
\hline dont pointe (fût) & (2) & \\
\hline dont pseudo baguette demi-ronde & (8) & \\
\hline dont indéterminé & (1) & \\
\hline Ebauche & 1 & \multirow{2}{*}{0,60} \\
\hline dont anneau ouvert & (1) & \\
\hline Fragments & 103 & 62,05 \\
\hline Total & 166 & \\
\hline
\end{tabular}

Tableau 11 - Bois de cervidés. Catégories techniques et fonctionnelles (y compris fragments de bois de renne sans trace a priori).

Table 11 - Antler. Technical and functionnal categories (with fragments maybe unworked).

certains bois de renne se pose de manière accrue lorsque l'on considère les très nombreux fragments $(n=103)$ qui ne portent pas de trace de travail a priori (fig. 24 ; graph. 2). De tels artefacts ont été signalés notamment dans le Badegoulien du Cuzoul de Vers. Sur ce site, une origine naturelle a été retenue pour rendre compte de leur état de fragmentation (Pétillon et Averbouh à paraître). En l'absence de référentiels naturalistes tels que ceux qui sont en cours d'élaboration sur les bois de renne de l'Igue du Gral (Castel et al. 2008), nous ne nous prononcerons pas sur le statut de ces fragments au Petit Cloup Barrat. Intégrés à l'inventaire, ils sont toutefois exclus du discours sur les techniques de segmentation des bois.

Plusieurs catégories techniques liées à l'exploitation de ces appendices frontaux ont été identifiées : des déchets de débitage (bases de bois de chute ou de massacre, cornillon, baguette brute, éclats), des déchets du façonnage (résections, baguettes régularisées), une ébauche et des objets finis. L'aspect lisse des bois et les rapports « tissu compact/tissu spongieux » conduisent à les attribuer au renne. C'est aussi à cette espèce que nous rapportons les objets finis, pour lesquels l'état de surface originelle a été effacé par les stigmates de fabrication.
Nous avons décompté neuf bases appartenant à huit bois de chute et à un bois de massacre (tabl. 12). La circonférence et les épaisseurs de tissu compact permettent de distinguer des bois de petits modules, munis d'un tissu compact de $4 \mathrm{~mm}$ d'épaisseur, rapportables à des femelles ou à de jeunes mâles. Les bois de modules supérieurs (moyens et gros modules), d'une épaisseur de compacta comprise entre 6 et $9 \mathrm{~mm}$, peuvent être attribués à des mâles adultes auxquels on pourrait joindre les différents fragments et éclats de $10 \mathrm{~mm}$ et plus (graph. 2) ${ }^{21}$.

\subsection{2 - Exploitation}

L'absence d'andouillers d'œil sur certaines des bases de bois de chute pourrait résulter d'un élagage réalisé par percussion lancée ou par entaillage. Les extrémités conservées des merrains ne portent pas de traces de travail a priori mais les états de surface sont, on l'a dit, moyennement conservés.

\section{Le rainurage}

C'est à partir d'autres fragments de merrains que l'on peut déterminer les méthodes et les techniques de segmentation des bois de renne. L'emploi du rainurage est attesté par huit fragments de baguettes brutes ou régularisées et de déchets de résection qui en portent les traces caractéristiques (fig. 25). L'objectif de ce débitage a été d'extraire des baguettes rectilignes de section carrée à

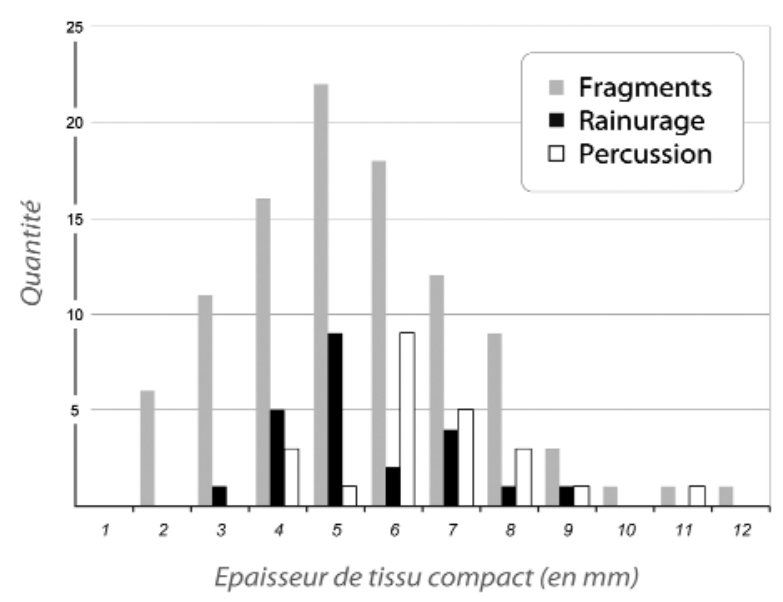

Graphique 2 - Bois de cervidés. Epaisseurs de tissu compact des fragments sans trace de travail, des produits issus du débitage par rainurage (déchets de fabrication et objets finis) et par percussion (éclats).

Graphe 2 - Antlers. Compacta thickness measurements of antler fragments maybe unworked, extracted products by double grooves (waste products and artifacts) and by percussion (flakes).

(21) En raison de la variabilité des modules des bois de renne selon le sexe et l'âge ainsi que de l'inconstance de l'épaisseur de tissu compact à l'intérieur d'un même bois, ces données chiffrées sont surtout indicatives (Pétillon et Averbouh, à paraître). 


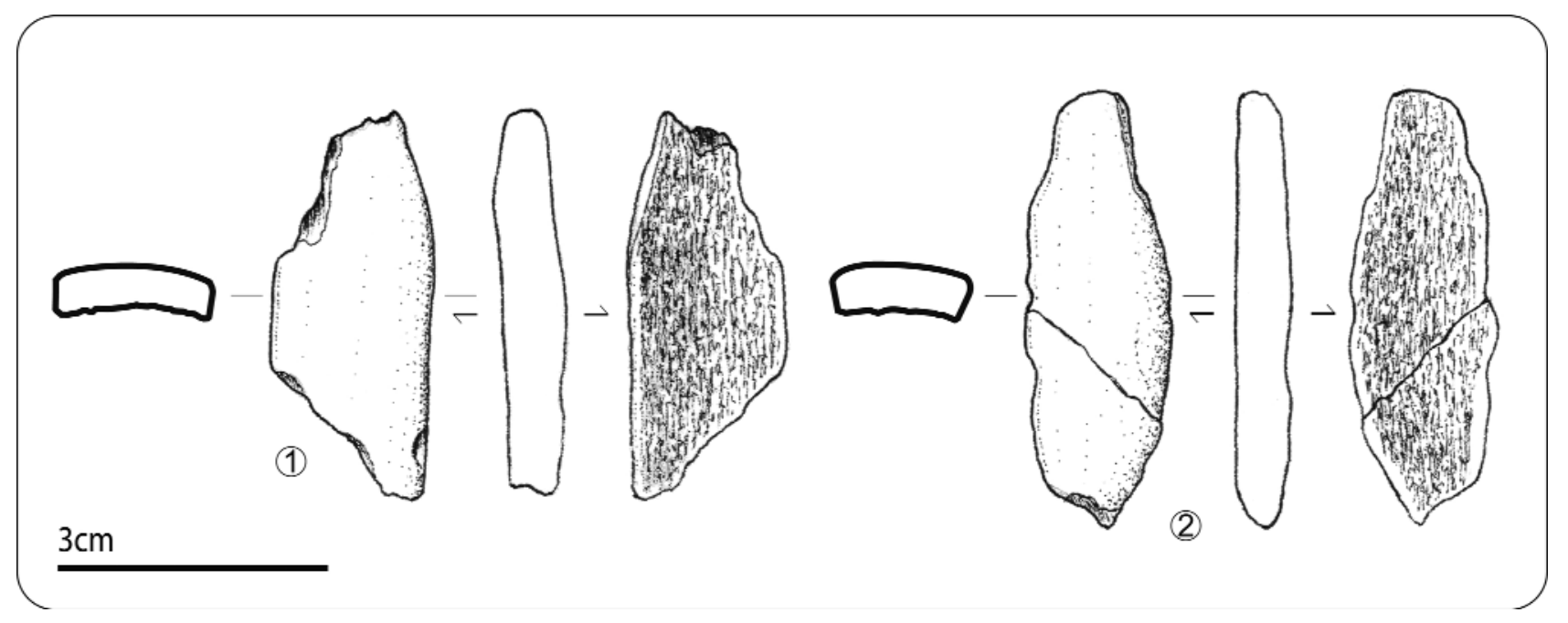

Figure 24 - Exemples de fragments de bois de renne sans trace de travail a priori. Dessins F.X. Chauviere.

Figure 24 - Fragments of Reindeer antler maybe unworked. Drawings F.X. Chauvière.

\begin{tabular}{|c|c|c|c|c|}
\hline Carré & Numéro d'inventaire & Nature du bois & Epaisseur du tissu compact (en mm) & Circonférence (en mm) \\
\hline P70A & 449 & chute & 6 & 65 \\
\hline P71 & 273 & chute & 8 & 135 \\
\hline P72B & 352 & chute & 9 & 120 \\
\hline Q70B & 33 & chute & 9 & 117 \\
\hline Q70B & 229 & massacre & 4 & 70 \\
\hline Q70B & 255 & chute & 4 & 80 \\
\hline Q70C & 112 & chute & 6 & 110 \\
\hline Q71C & $132-133$ & chute & 8 & 133 \\
\hline R71D & 359 & 8 & 100 \\
\hline
\end{tabular}

Tableau 12 - Bois de chute et de massacre. Circonférence et épaisseurs de tissu compact.

Table 12 - Circumference and compacta thickness measurements of shed and unshed antler bases.

rectangulaire, peu larges (fig. 25, $\left.n^{\circ} 1-5\right)$. Après un premier façonnage par raclage qui a régularisé les baguettes (fig. 25, $n^{\circ} 6-7$ ), celles-ci ont été mises à l'axe et au gabarit par segmentation transversale d'une ou des deux extrémités, selon des modalités déjà décrites par ailleurs (Chauvière et Rigaud 2005). On retrouve un témoin de cette opération sous la forme d'un déchet de résection pointu, obtenu sans doute par percussion pour la segmentation transversale du volume (fig. $25, n^{\circ} 8$ ).

L'objectif de cette mise à façon peut être perçu au travers de pointes de petit calibre que l'on peut interpréter comme des têtes de projectile (fig. 26). La couche 4 a livré sept fragments de pointes, de section ronde $(n=6)$ ou elliptique $(n=1)$. Une seule pièce porte une incision que l'on peut assimiler à une discrète ornementation (fig. 26, $\mathrm{n}^{\circ}$ 2). Nous pensons pouvoir identifier une pointe de type LussacAngles (fig. 26, $n^{\circ} 4$ ) dont un autre exemple existe par ailleurs dans le site et dans d'autres séries régionales, comme dans les couches III et C de Sainte-Eulalie notamment (Lorblanchet 1972 ; Lorblanchet et coll. 1973 ; Castel et al. à paraître).

C'est aussi probablement à partir de baguettes extraites par rainurage que huit fragments de « pseudo baguettes demi-rondes ${ }^{22}$ ont été fabriquées (fig. $26, n^{\circ} 6-13$ ). Si elles sont effectivement de section semi circulaire, ces pièces ne présentent pas les traitements de surface appliqués sur les tissu spongieux (stries d'encollage) ou compact (incisions) des faces inférieure et supérieure des baguettes

(22) Selon la terminologie employée par J.-M. Pétillon pour la couche B de la grotte de Scilles (Lespugue, Haute-Garonne : Pétillon et al. 2008 ; Langlais et al. 2010). 

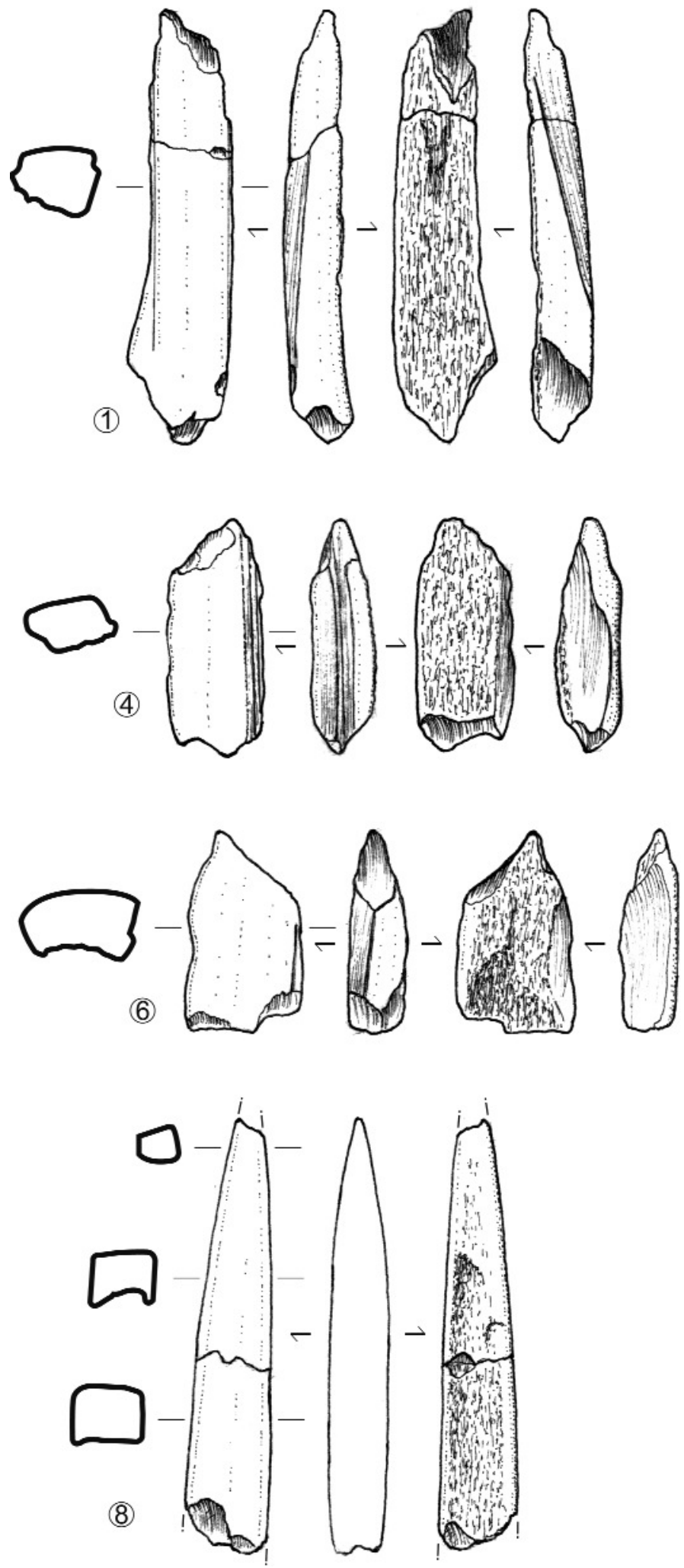
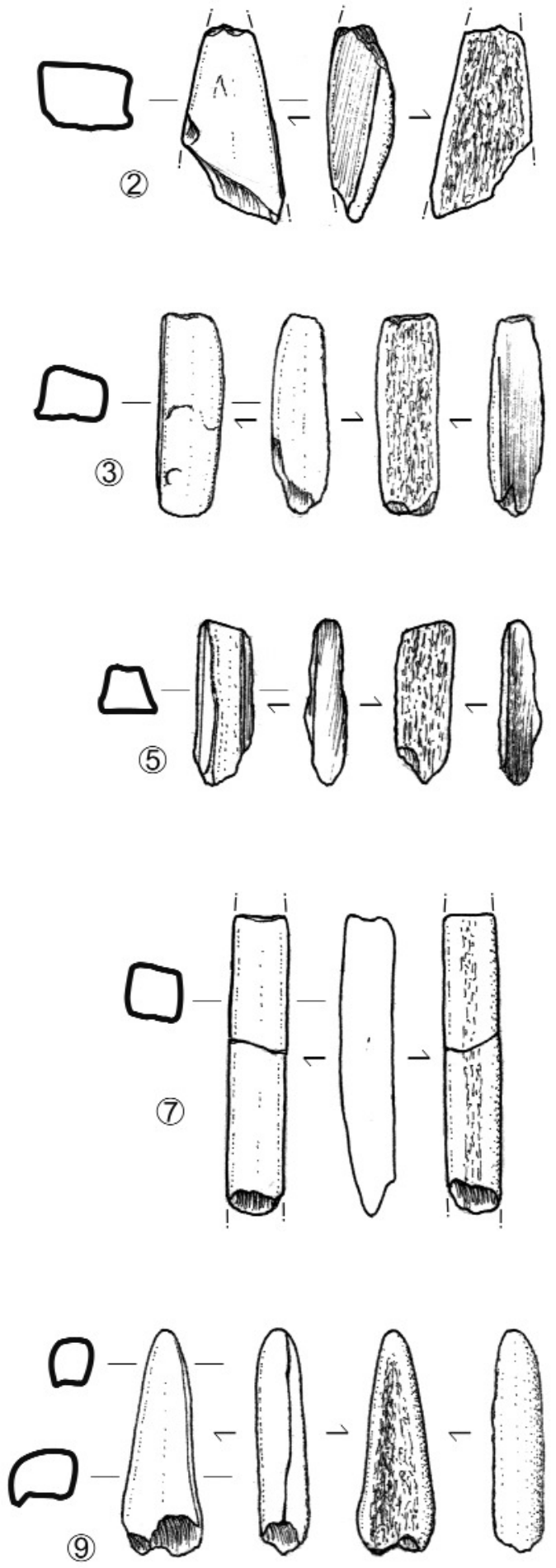

$3 \mathrm{~cm}$

Figure 25 - Produits du débitage par rainurage des bois de cervidés ( $n^{\circ} 1$ à $5:$ baguettes brutes, $n^{\circ} 6$ et $7:$ baguettes régularisées, $n^{\circ} 8$ : déchet de résection). Dessins F.-X. Chauvière.

Figure 25 - Reindeer Antler industry. Extracted products by double grooves ( $n^{\circ} 1-6$ : unformed baguettes, $n^{\circ} 7,8$ : formed baguettes, $n^{\circ}$ 9: waste of "resection ». Drawings F.X. Chauvière. 

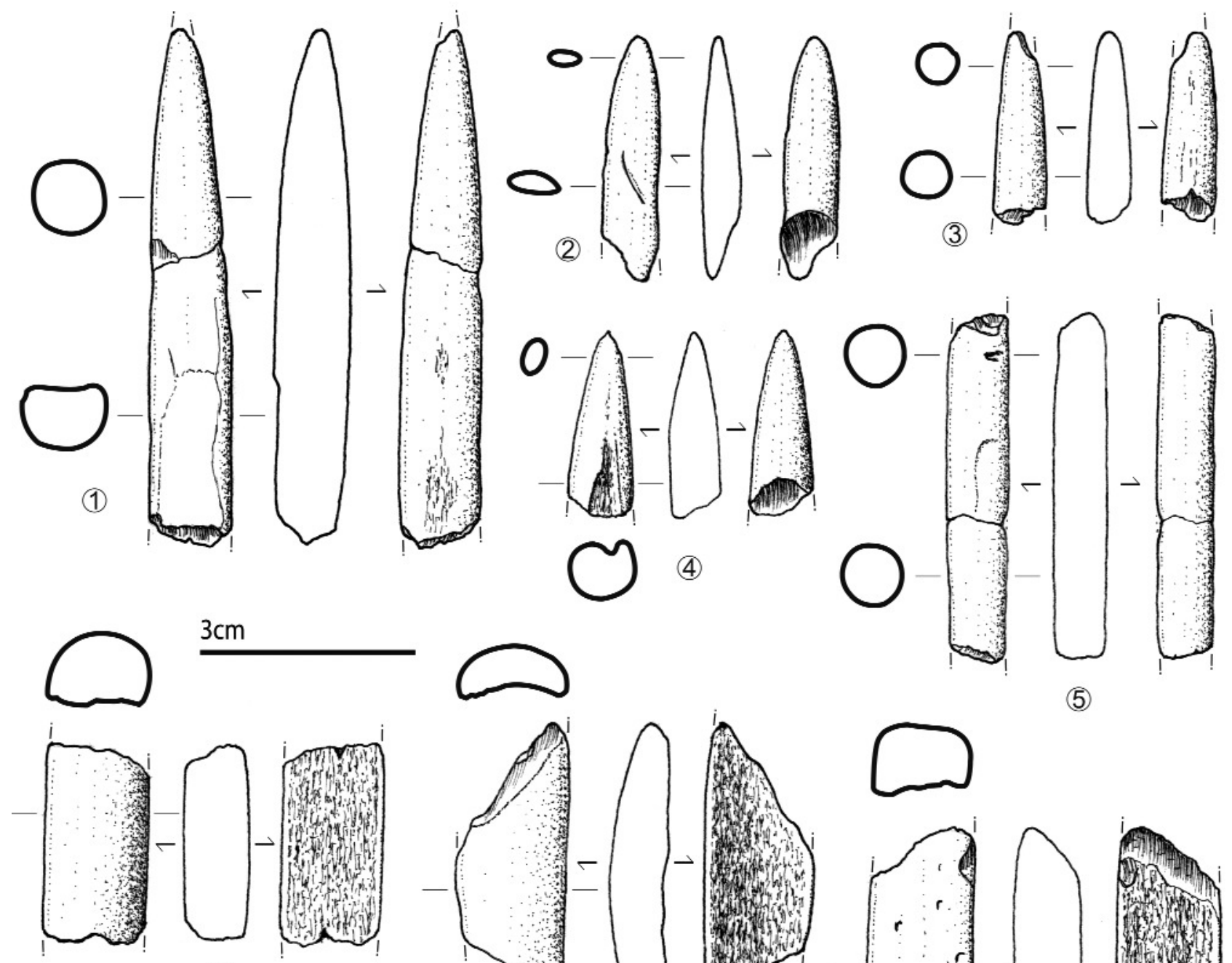

(6)
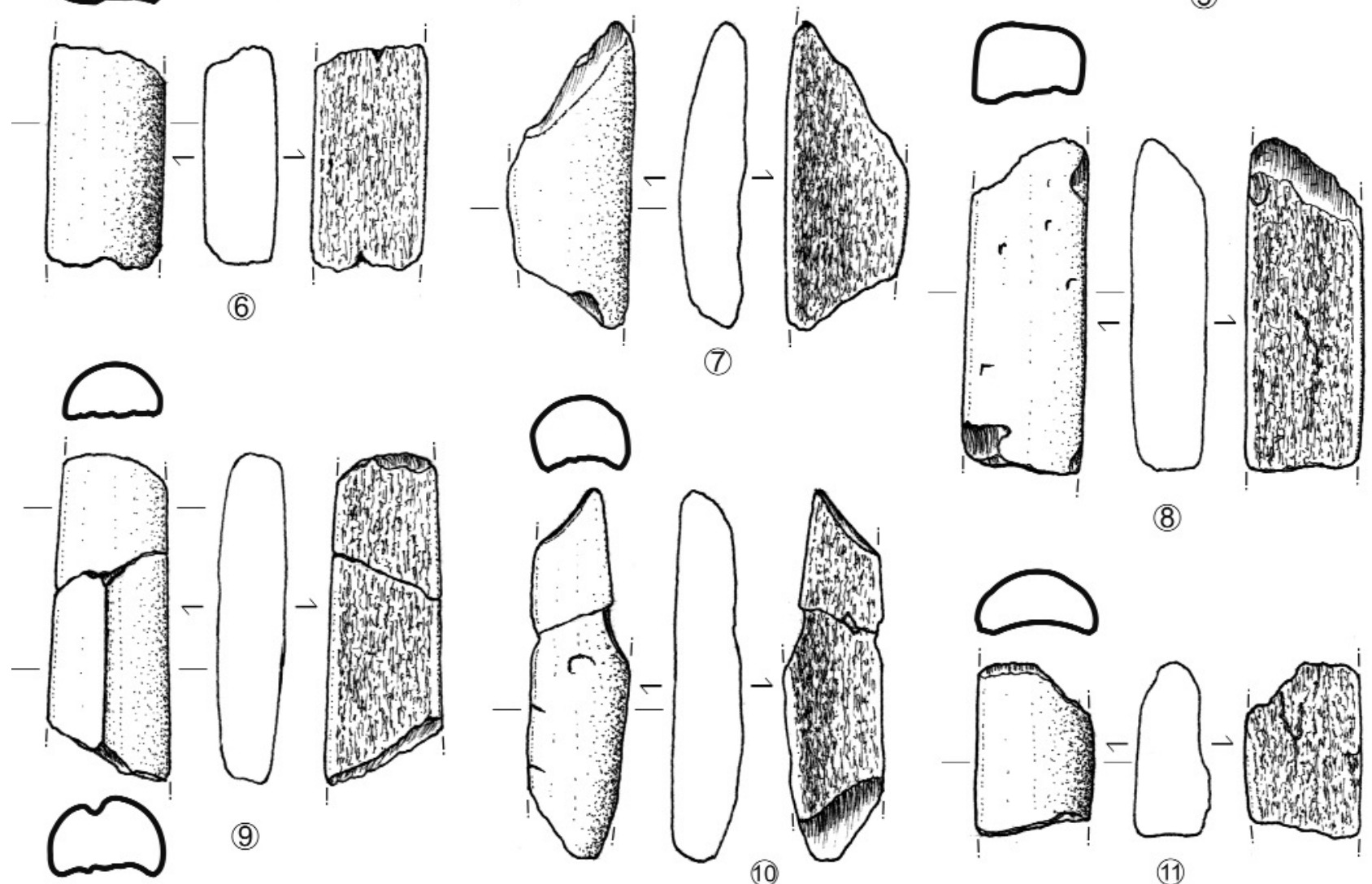

(8)

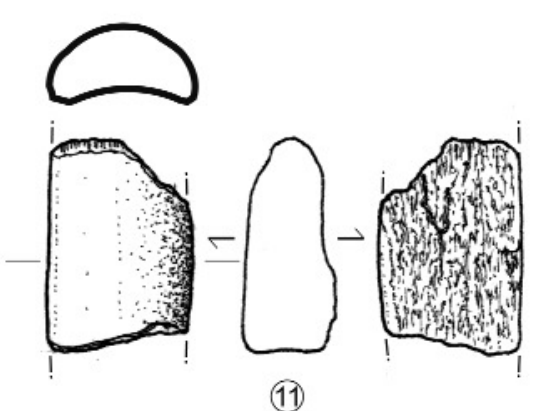

Figure 26 - Objets finis façonnés sur baguettes ( $n^{\circ} 1$ à 5 : pointes de projectile, $n^{\circ} 6$ à 13 : pseudo baguettes demi-ronde). Dessins F.-X. Chauvière.

Figure 26 - Reindeer Antler industry. Manufactured artifacts ( $n^{\circ}$ 1-5: projectile points, $n^{\circ}$ 6-11: baguettes « demi-ronde »). Drawings F.X. Chauvière. 
demi-rondes vraies de la phase moyenne du Magdalénien (Feruglio 1992). Le statut fonctionnel de ces pseudo baguettes demi-rondes, signalées par ailleurs dans la couche B la grotte des Scilles (Pétillon et al. 2008 ; Langlais et al. 2010a), n'est pas défini pour l'instant.

La mise en regard des épaisseurs de tissu compact et des largeurs des déchets de fabrication/résection et des objets finis ainsi que des bases laisse entrevoir une exploitation discrète des bois de petits modules alors qu'elle apparaît plus marquée pour les bois de moyen et gros modules (graph. 3). II est possible d'établir notamment une corrélation entre tous les éléments issus du rainurage.

\section{La percussion lancée}

D'autres fragments de bois de renne portent les stigmates caractéristiques d'une segmentation par percussion lancée avec un percuteur (fig. 27, $n^{\circ} 1-4$ ), outil dont des exemplaires ont été identifiés par ailleurs dans la couche 4 (Daulny 2004, 2005). C'est plutôt à l'exploitation de bois de gros modules qu'il faut rapporter ces éclats obtenus par percussion (graph. 3).

La diversité morphométrique de ces éclats $(n=23)$ et l'absence actuelles d'ébauches ou d'objets finis comparables aux pointes épaisses de section ovale découvertes à l'abri Fritsch (Indre) (Allain et al. 1974 ; Rigaud 2004) et au Cuzoul de Vers (Lot) (Pétillon et Averbouh à paraître) ne permettent pas de saisir les objectifs de cette méthode de fragmentation des volumes mise en œuvre dans la couche 4 du Petit Cloup Barrat. Toutefois, nous interprétons un éclat façonné comme la résection d'une ébauche mise au gabarit et à l'axe non retrouvée dans la couche 4 (fig. $27, n^{\circ} 5$ ).

\section{Une ébauche d'anneau ouvert}

Parmi le corpus d'objets fabriqués en bois de renne de la couche 4 du Petit Cloup Barrat, il convient de s'attarder sur une ébauche d'anneau ouvert (ou objet arciforme ou en forme d'oméga) (fig. 28). Sur cette pièce réalisée sur un fragment de merrain, le contour semi-annulaire est déjà bien marqué, tout comme l'est l'un des deux épaulements latéraux caractéristiques de ce type d'objet dont on ne connaît pas le mode de fonctionnement (Feruglio et LeroyProst 2001). La perforation n'est qu'ébauchée : un grattage profond est visible sur les deux faces de la pièce. Poussée à son maximum, cette opération aurait fini par ouvrir totalement l'anneau à sa base.

Dans la littérature, onze autres exemplaires de cette catégorie d'objets sont signalés, groupe auquel la pièce du Petit Cloup Barrat s'intègre parfaitement (graph. 4). Dix proviennent des fouilles anciennes de la grotte du Placard (Vilhonneur, Charente), le dernier faisant partie de la collection de la "Cave à Endives" (Cabrerets, Lot). Les objets mis au jour dans le premier site appartiennent à des niveaux magdaléniens non définis (Feruglio et Leroy-Prost op. cit). Pour le second, il n'existe aucun contexte stratigraphique connu (Raux 1994 ; Raux et PielDesruisseaux 1997). Dès lors, on conçoit toute l'importance de la pièce du Petit Cloup Barrat car c'est la seule du Paléolithique supérieur à bénéficier d'un contexte stratigraphique vérifié. II s'agit également d'un artefact qui permet de confirmer les liens entre Charente et Quercy. Ceux-ci ont déjà été envisagés par le biais de l'anneau ouvert décoré de la Cave à Endives et, dans la diachronie, au travers des signes pariétaux de type Placard identifiés dans le site éponyme ainsi que dans les grottes du Pech Merle et de Cougnac (Clottes et al. 1990).

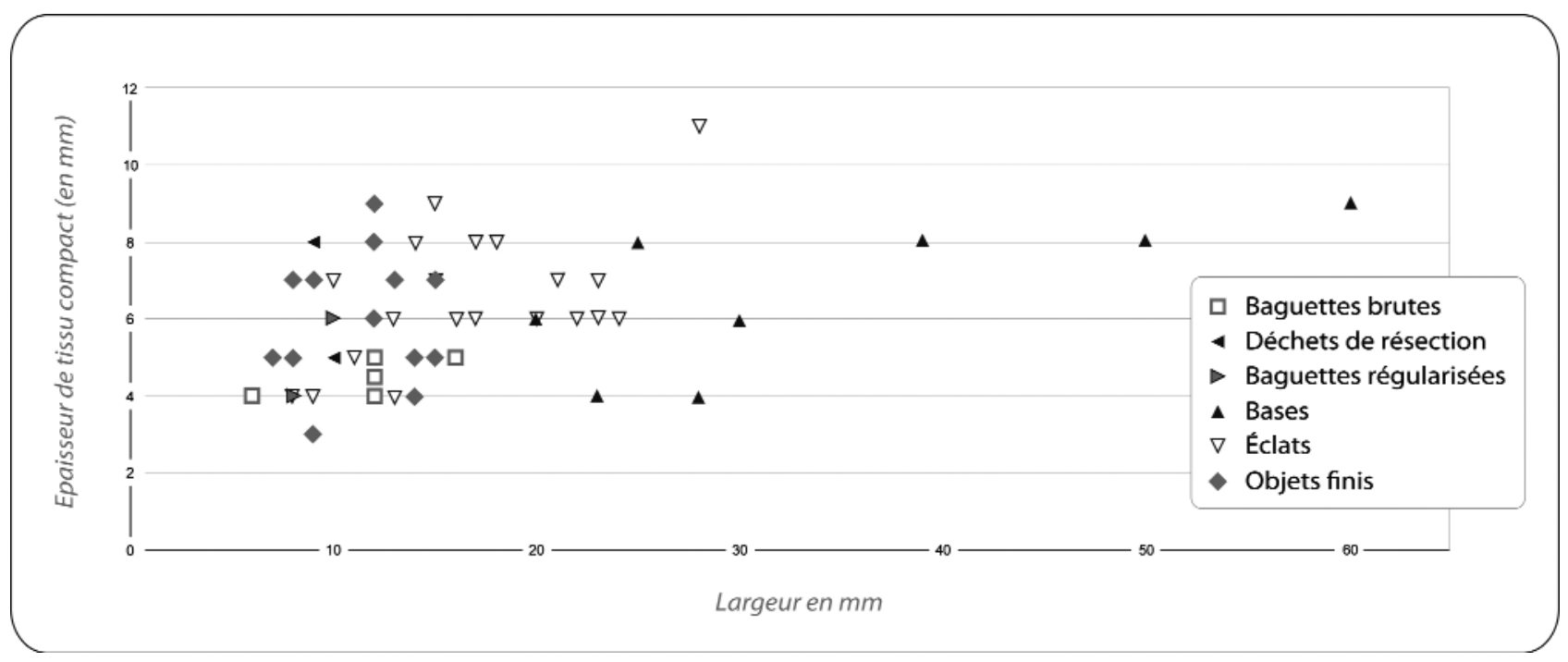

Graphique 3 - Bois de cervidés. Largeur et épaisseur des produits issus du débitage par rainurage (déchets de fabrication et objets finis) et par percussion (éclats).

Graphe 3 - Antlers. Length and width measurements of extracted products by double grooves (waste products and artifacts) and by percussion (flakes). 


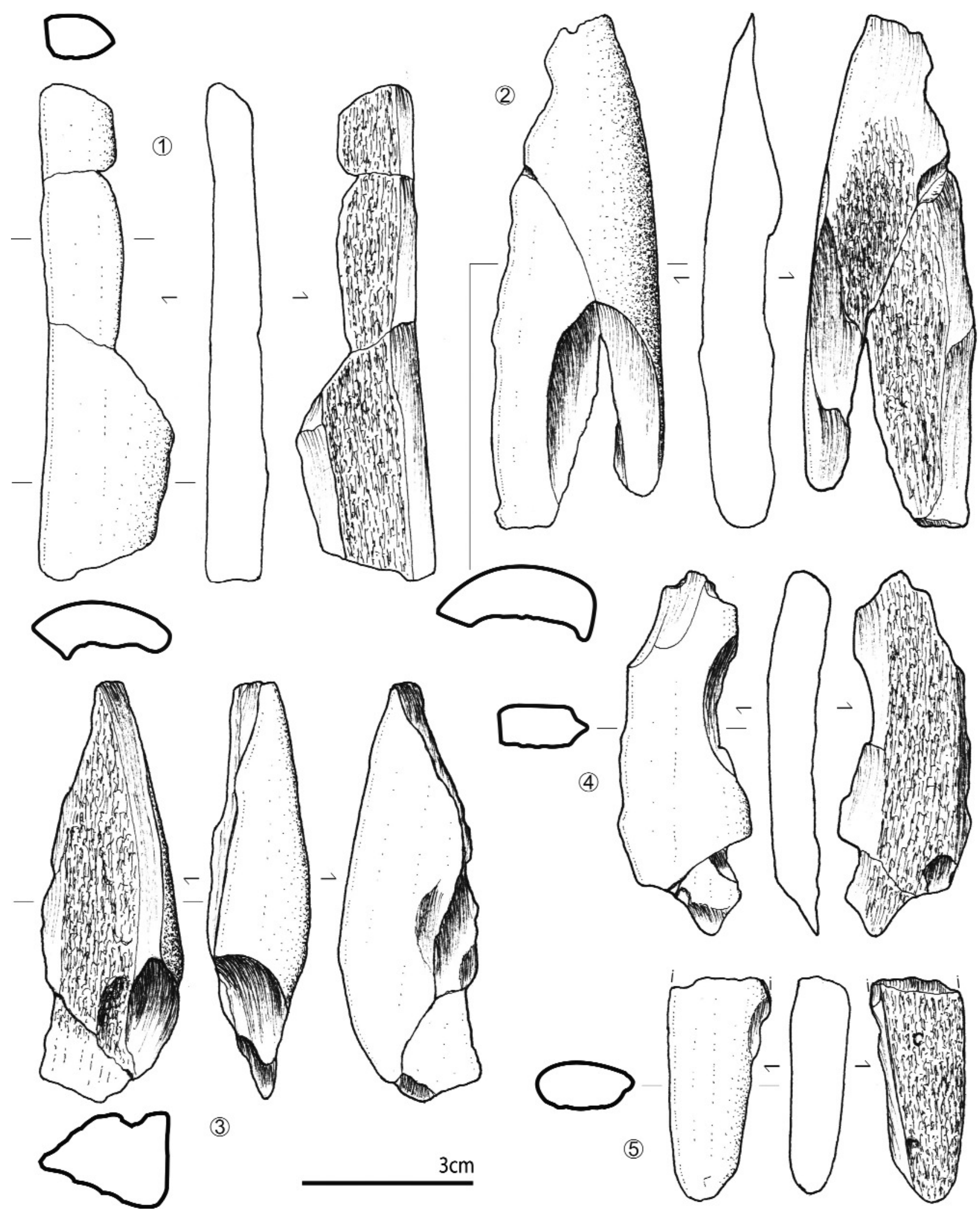

Figure 27 - Produits du débitage par percussion des bois de cervidés ( $n^{\circ} 1$ à 4 : éclats, $n^{\circ} 5$ : déchet de résection). Dessins F.-X. Chauvière.

Figure 27 - Reindeer Antler industry. Products by percussion ( $n^{\circ}$ 1-4: flakes, $n^{\circ}$ 5: waste of « résection »). Drawings F.X. Chauvière. 


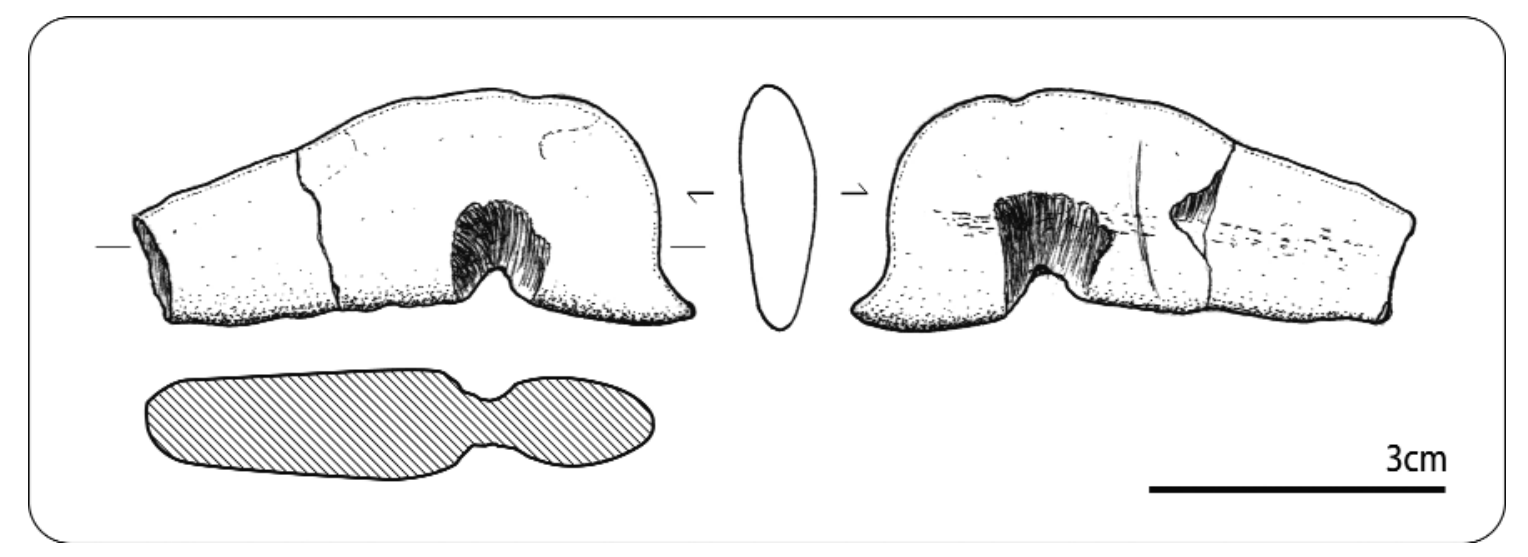

Figure 28 - Ebauche d'anneau ouvert. Dessin F.-X. Chauvière.

Figure 28 - Reindeer antler. Preform of opened ring. Drawing F.-X. Chauvière.

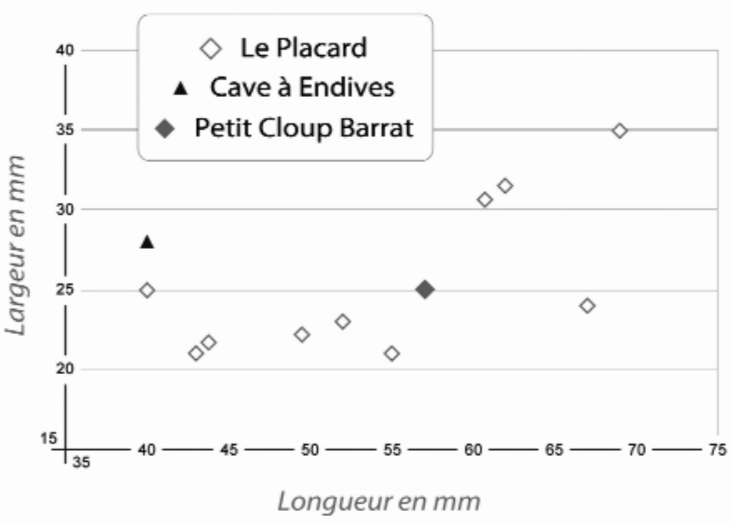

Graphique 4 - Longueur et largeur des anneaux ouverts en bois de renne du Paléolithique supérieur.

Graphe 4 - Length and width measurements of Upper

Palaeolithic open rings made with reindeer antler.

\section{2 - Le travail de l'os}

La transformation de cette matière est attestée par dix éléments, essentiellement des fragments mésiaux d'aiguilles à chas (tab. 13 ; fig. 29). Un seul témoin de la confection sur place de ces objets perforants a été identifié. II peut être interprété comme un déchet de résection d'une baguette rectiligne extraite de la matrice osseuse par double rainurage. II existe également un (deux?) affûtoir(s) (au sens de Rigaud 2007) sur diaphyse d'os long d'ongulé de taille moyenne et une petite perle tubulaire $(8 \times 5 \times 5 \mathrm{~mm})$, totalement brûlée.

\section{3 - Dents et coquilles : la parure corporelle}

D'une manière générale, les différents niveaux archéologiques du Petit Cloup Barrat ont livré une quantité déjà non négligeable d'incisives d'herbivores sciées au

\begin{tabular}{|c|c|}
\hline & Nombre \\
\hline Aiguille à chas & 6 \\
\hline dont objet fini & (4) \\
\hline dont ébauche & (1) \\
\hline dont déchet defabrication & (1) \\
\hline Affûtoir & 2 \\
\hline Parure & 1 \\
\hline Indéterminé & 1 \\
\hline Total & 10 \\
\hline
\end{tabular}

Tableau 13 - Os. Catégories techniques et fonctionnelles.

Table 13 - Worked bones. Technical and functionnal categories.

trait de silex à la racine, en face vestibulaire. La couche 4, quant à elle, en a fourni 17, parmi lesquelles huit couronnes constituent des éléments utilisés dans la parure corporelle selon des modalités initialement présentées par ailleurs (Poplin 1983) (tabl. 14 ; fig. 30A, $n^{\circ} 1$ à 7). La présence de neuf racines «sciées », véritables déchets de fabrication, argumente la production de cette parure sur place, qui est immédiatement consécutive à l'abattage des animaux pour des raisons de conservation du tissu gingival qui maintient les dents (fig. 30A, $n^{\circ} 8$ à 15) (Chauvière 2006). La parure de la couche 4 du Petit Cloup Barrat se singularise également par la rareté des dents percées, au nombre de deux seulement. II s'agit d'incisives de renne dont l'une, entière, est perforée à la racine (fig. 30A, $n^{\circ} 16$ ). L'autre a été percée puis sciée à la racine selon des modalités que l'on identifie sur de très nombreux exemplaires des sites de la vallée de l'Aveyron (Ladier et Welté 1993, 1994) (fig. 30A, $n^{\circ} 17$ ). 
Q-

(1) o-

(2)<smiles>O=C1CCCC1</smiles>

(3)<smiles>O=[Ti]=[Ti]</smiles>

(4)

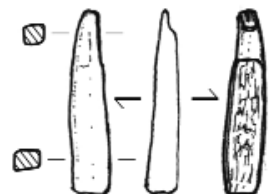

(5)
$\mathbb{Q}-]_{!}^{i}$

(6)

Figure 29 - Objets en os. Dessins F.-X. Chauvière.

Figure 29 - Bone artifacts ( $n^{\circ}$ 1-4: needles, $n^{\circ} 5$ : waste of needle, $n^{\circ} 6$ : cylindrical bead). Drawings F. $X$. Chauvière.

\begin{tabular}{|c|c|c|c|}
\hline & Renne & PMM & Total \\
\hline Couronne d'incisive sciée & 8 & & 8 \\
\hline Racine d'incisive sciée & & 9 & 9 \\
\hline Incisive percée et sciée & 1 & & 1 \\
\hline Incisive percée & 1 & & 1 \\
\hline Total & 10 & 9 & 19 \\
\hline
\end{tabular}

Tableau 14 - Dent - Déterminations anatomique, spécifique et catégories techniques et fonctionnelles.

Table 14 - Teeth. Anatomical and specific attributions, technical and functionnal categories.

A l'inverse de ce que l'on a pu observer ou suspecter dans les couches badegouliennes du Cuzoul de Vers (Castel 2003, 2010), des Peyrugues (Juillard 2009) ou dans le Magdalénien de la Bergerie-des-Quatre-Chemins (SéronieVivien et al. 1981), il n'y a pas de surreprésentation d'incisives d'herbivores travaillées ou non dans la couche 4 du Petit Cloup Barrat. L'acquisition des dents sciées s'est donc probablement faite au détriment d'un « stock » faunique similaire à celui qui est attesté dans le site.
Enfin, à cette parure sur dents d'herbivores sont associés des ornements en coquillages. Si le Petit Cloup Barrat en a livré plus d'une quarantaine, seuls trois exemplaires de Dentalium $\mathrm{sp}$. de petites dimensions ont été retenus comme étant parfaitement intégrés à la couche 4 (fig. 30B).

\section{4 - Conclusion}

Au-delà des morphologies et des catégories fonctionnelles qui sont communes à d'autres techno-complexes du Paléolithique supérieur (aiguilles à chas, pointes de projectiles), l'industrie osseuse de la couche 4 du Petit Cloup Barrat, de par ses particularités, permet d'insister plus encore sur la variabilité des systèmes techniques liés à l'exploitation des matières dures d'origine animale au cours du Dernier Maximum Glaciaire (tabl. 15).

En premier lieu, mentionnons la coexistence des deux principaux modes de fragmentation du bois de renne au sein de la couche 4, à savoir le rainurage et l'éclatement par percussion. Actuellement, il n'y a pas de critères pour contester cette association. En ce sens, le Petit Cloup Barrat se distingue des assemblages du Badegoulien et du Magdalénien inférieur dans lesquels cette mixité n'existe pas (Pétillon et al. 2008 ; Langlais et al. 2010a ; Primault

\begin{tabular}{|c|c|c|c|c|c|c|c|}
\hline & & $\begin{array}{c}\text { Petit Cloup Barrat c.4 } \\
\text { (Cabrerets, Lot) }\end{array}$ & $\begin{array}{l}\text { Les Scilles couche B } \\
\text { (Lespugue, Haute-Garonne) }\end{array}$ & $\begin{array}{l}\text { St Germain-la-Rivière c.3 et } \mathbf{4} \\
\text { (St Germain-la-Rivière, Gironde) }\end{array}$ & $\begin{array}{c}\text { Le Taillis des Coteaux AG-III/EG-III } \\
\text { (Antigny, Vienne) }\end{array}$ & $\begin{array}{l}\text { Abri Gandil c.20, 23-25 } \\
\text { (Bruniquel, Tam-et-Garonne) }\end{array}$ & $\begin{array}{c}\text { Lassac locus } 1 \\
\text { (Sallìles-Cabardès, Aude) }\end{array}$ \\
\hline \multirow{7}{*}{ 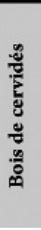 } & rainurage & oui & oui & oui & oui & oui & \\
\hline & percussion & oui & & & & & oui \\
\hline & pointe & 6 & 29 & oui & oui & 32 & oui \\
\hline & pseudo baguette demi-ronde & 5 & 3 & & & & \\
\hline & outil intermédiaire & & 7 & oui & & & oui \\
\hline & anneau ouvert & 1 & & & & & \\
\hline & bâton percé & & & oui & & & \\
\hline \multirow{4}{*}{8} & aiguille à chas & 4 & 1 & & oui & 3 & oui \\
\hline & affûtoir & 1 & 7 & & & & \\
\hline & outil intermédiaire & & 2 & & & & \\
\hline & pendeloque & 1 & 1 & & & & \\
\hline \multirow{4}{*}{ ڤ̆ } & sciée & 8 & & & & 1 & \\
\hline & incisée & & 2 & & & & \\
\hline & percée & 1 & & & & 2 & \\
\hline & percée et sciée & 1 & & & & & \\
\hline \multirow{2}{*}{ 咅 } & percée & & 6 & & & & \\
\hline & sciée & 3 & & & oui & & \\
\hline & Références & $\begin{array}{c}\text { Castel et al. } 2006 \\
\text { Chauviere et al. } 2008\end{array}$ & $\begin{array}{l}\text { Langlais et al. } 2010 \\
\text { Pétillon et al. } 2008\end{array}$ & Lenoir et al. 1991 & $\begin{array}{c}\text { Primault et al. } 2007 \text {; Primault, } 2010 \\
\text { Houmard com. pers. }\end{array}$ & $\begin{array}{l}\text { Langlais et al. } 2007 \\
\quad \text { Ladier } 2000\end{array}$ & $\begin{array}{l}\text { Pétillon et Sacchi, à } \\
\text { paraître }\end{array}$ \\
\hline
\end{tabular}

Tableau 15 - Industries sur matières dures d'origine animale de quelques sites attribués au Magdalénien inférieur et au Badegoulien.

Table 15 - Osseous industries of several Lower Magdalenian and Badegoulian sites. 


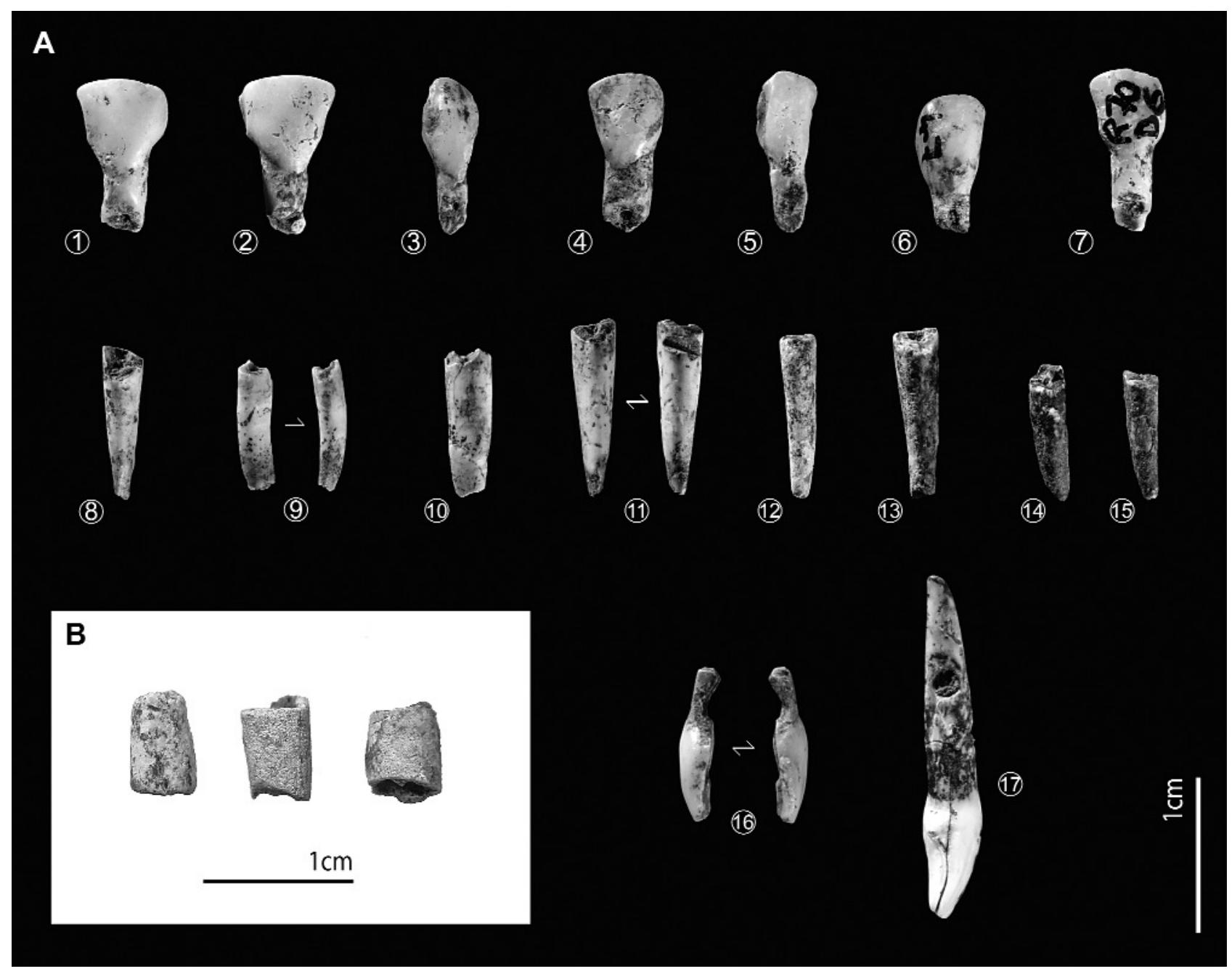

Figure 30 - $A$ - Les dents travaillées ( $n^{\circ} 1$ à 7 : couronnes sciées, $n^{\circ} 8$ à 15 : racines sciées, $n^{\circ} 16$ : incisive de renne percée et sciée, $n^{\circ} 17$ : incisive de renne percée). Clichés Ph. Wagneur. B - Coquilles de dentalium sp. Clichés J.-C. Castel.

Figure 30 - $A$ - Worked teeth ( $n^{\circ}$ 1-7: sawed teeth crowns, $n^{\circ}$ 8-15: sawed teeth roothes, $n^{\circ}$ 16- perforated and sawed reindeer incisor, $n^{\circ}$ 17: perforated reindeer incisor). Photos Ph. Wagneur. B- Shells of Dentalium sp. Photographies J.-C. Castel.

2010 ; Pétillon et Averbouh à paraître ; Pétillon et Sacchi à paraître).

La présence conjointe de pointes de projectile, de pseudo baguettes demi-rondes, déjà constatée dans le Magdalénien inférieur de la grotte des Scilles (Pétillon et al. 2008 ; Langlais et al. 2010a), est ici confirmée. En revanche, on note l'absence d'outils intermédiaires de type ciseau au Petit Cloup Barrat alors qu'ils sont signalés aux Scilles, à Saint-Germain-la-Rivière (Lenoir et al. 1991 ; Pétillon et al. op. cit. ; Langlais et al. op. cit.) ainsi qu'au Cuzoul de Vers (Pétillon et Averbouh à paraître). Enfin, la présence marquée des incisives d'herbivores sectionnées à la racine et celle d'une ébauche d'anneau ouvert rendent originale la série tout en ajoutant à son intérêt pour le calage chronologique de ces types d'objets.

\section{6 - Pour une mise en perspective globale (SD, FXC, ML et JCC)}

Au terme de ces différentes analyses, et malgré le caractère provisoire de l'échantillon, se dessine l'image d'une industrie vraisemblablement homogène et résolument originale. Mais nous allons voir que cette originalité alimente des discussions de plus en plus étayées sur les relations existant entre les mondes badegouliens et magdaléniens (Bodu et al. dir. 2007 ; Langlais 2007 ; Pétillon et al. 2008 ; Ducasse 2010).

En l'état actuel des données, et en dehors de toute réflexion d'ordre chrono-culturel, le statut fonctionnel des occupations de la couche 4 est difficile à cerner. Si nous ne perdons pas de vue le fait que cet ensemble puisse correspondre à une accumulation d'activités (et donc 
témoigner d'une certaine diachronie), la coexistence d'armes de chasse (en bois de renne et en silex), d'outils domestiques (en os et en silex) et d'éléments de parure parfois réalisés sur place ${ }^{23}$ semble s'éloigner de l'idée que le Cloup Barrat ait pu tenir lieu, dans ce cas précis, de " halte de chasse » spécialisée dans l'acquisition de grands herbivores fréquentant le causse. La complémentarité économique entre occupations de vallées et exploitation des plateaux ne se vérifie donc pas à travers l'analyse de la couche 4 puisque, contrairement aux couches 8 et 9 de la grotte voisine de Pégourié qui font la part belle aux bovinés (Séronie-Vivien 1995, p. 199-200), le type de chasse identifié au Cloup Barrat ne dépareille pas de ce qui est connu tant au Cuzoul de Vers que dans la plupart des sites magdaléniens de la vallée du Lot. Pour une même saison d'occupation (entre l'été et la fin de la bonne saison), tous sont en effet marqués par l'acquisition dominante du Renne (Castel et al. à paraître).

Arguments pour une attribution à la phase ancienne du Magdalénien

L'acquisition et l'exploitation du gibier a donné lieu à la confection et/ou la réfection d'un équipement lithique et osseux dont les modalités de fabrication ainsi que la typologie définissent un ensemble d'une grande cohérence, ensemble rappelant fortement les industries contemporaines de la seconde moitié du DMG, soit entre 17500 et 15000 BP (20500-18000 cal. BP ; GS2b).

Les industries en silex, structurées autour d'un binôme « lame/lamelles » répondant dans les grandes lignes à une dichotomie "domestique/cynégétique ", laissent une place non négligeable aux éclats qui, de leur côté, jouent un double rôle. Pour partie intégrés à l'équipement de transformation (production autonome d'éclats minces ; récupérations de sous-produits lamino-lamellaires), ils sont également à l'origine d'une ramification des chaînes opératoires : la recherche de lamelles, composante essentielle de l'industrie de la couche 4 , est fréquemment liée à l'exploitation d'éclats épais ou allongés selon une grande diversité de modalités. Cette diversité, qui témoigne dans certains cas d'une pluralité d'objectifs, s'exprime à travers la mise en œuvre de schémas opératoires dont certains ne sont actuellement rencontrés qu'au sein d'assemblages attribués au Magdalénien inférieur. Ainsi, par exemple, l'obtention de supports microlamellaires à partir de nucléus dits " sur front ventral déjeté » (cf. supra) rappelle les industries de l'abri Gandil (Tarn et Garonne ; couches 20, 23/25), de Saint-Germainla-Rivière (Gironde ; couches 3 et 4), de Thèmes (Yonne ; où il a été décrit pour la première fois : Le Brun-Ricalens et Brou 2003), des Scilles (Haute-Garonne; couche B) ou de Fontgrasse (Gard ; Bazile 1989), trouvant même des échos jusqu'en péninsule ibérique (Erralla V : Cazals 2000 et Langlais 2007, Cabeço de Poto Marinho : Marks et Mishoe 1997).
En dehors de la présence de microlamelles à dos, documentée dans des contextes variés attribués au Badegoulien (Bodu et al. 2007 ; Ducasse 2004 et 2010), au Magdalénien inférieur (Langlais 2007) où à l'Epigravettien ancien (C. Montoya com. orale), c'est avant tout l'existence du morphotype particulier que représentent les lamelles à dos dextre marginal que les liens entre la plupart de ces gisements semblent se resserrer (fig. 31) : clairement identifiées à Saint-Germain-la-Rivière, au Taillis des Coteaux (AG-Illc : Primault et al. 2010 ; A. Gioé Master 1 en cours à l'Université de Toulouse 2), aux Scilles (Pétillon et al. 2008) et à Lascaux (Allain 1979 ; Glory 2008 ; Langlais 2010, p. 275-276), elles semblent s'inscrire dans un intervalle chronologique compris entre 17500 et 16000 BP selon les datations AMS disponibles actuellement, succédant directement aux industries les plus récentes du techno-complexe Badegoulien.

Les industries osseuses, de leur côté, affermissent cette proposition, non seulement d'un point de vue technique, mais aussi typologique : les pointes osseuses de section ronde/elliptique et les "pseudo baguette demirondes " (Pétillon et al. 2008) ont vraisemblablement été façonnées à partir de supports obtenus par la technique du double rainurage a priori absente de la panoplie technique badegoulienne (Pétillon et Averbouh à paraître, Pétillon et Sacchi à paraitre, Pétillon et Ducasse en préparation). II ne manquerait, pour parfaire cet l'inventaire, qu'une pointe de sagaie à trois rainures, objet qui semble fréquent au Magdalénien inférieur : en effet, les exemplaires connus et " contextualisés " $^{24}$ issus du niveau AG-Illc du Taillis des Coteaux (2 exemplaires : Primault 2010) ainsi que de la couche B des Scilles (1 exemplaire : Pétillon et al. 2008), laissent à penser, au vu de la particularité de ces aménagements, qu'il s'agit peut-être d'un « marqueur » chrono-culturel à tester. Dès lors, la découverte d'une pointe présentant ce type de modifications au sein de la collection de la "Cave à Endives " dont les liens avec la couche 4 du Petit Cloup Barrat nous semblent extrêmement étroits (fig. 32, cf. supra), constituerait un argument supplémentaire dans la perspective d'une attribution culturelle de l'industrie osseuse de la couche 4 au Magdalénien inférieur.

Si le lot d'incisives d'herbivores sciées, étroitement associé à l'ensemble du matériel issu de la couche 4, trouve des parallèles au Magdalénien supérieur (Poplin 1983 ; Bonnissent et Chauvière 1999 ; Berganza et Arribas 2010), les petits fragments de Dentalium sp., malgré leur ubiquité, rappellent les exemplaires calibrés signalés dans le Magdalénien inférieur du Taillis des Coteaux (Primault 2010, p. 285 ; Peschaux 2009 et 2010).

L'ensemble de ces éléments nous incite donc à privilégier une attribution de l'industrie de la couche 4 au Magdalénien inférieur, proposition étayée dès la campagne 2004 (Langlais et al. 2004). Néanmoins, la

(23) En excluant l'ébauche d'anneau ouvert dont le statut fonctionnel reste à ce jour inconnu.

(24) Nous excluons ici l'exemplaire décrit à Gandil au vu de sa provenance stratigraphique incertaine (collection Chaillot : Langlais 2010). 

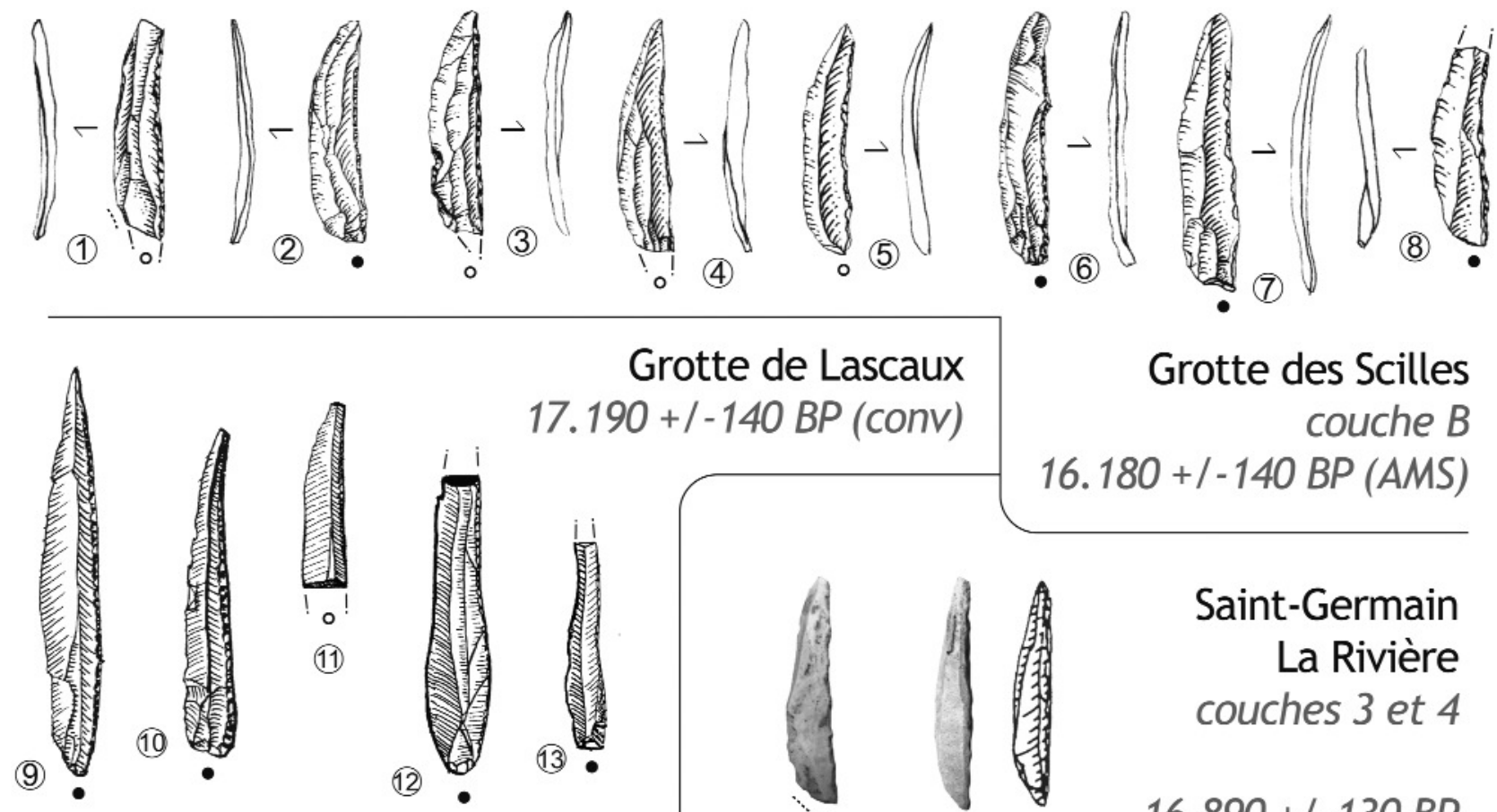

$17.190+/-140$ BP (conv)

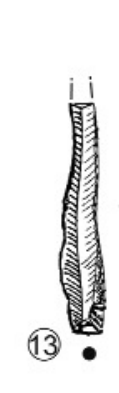

Grotte de Lascaux

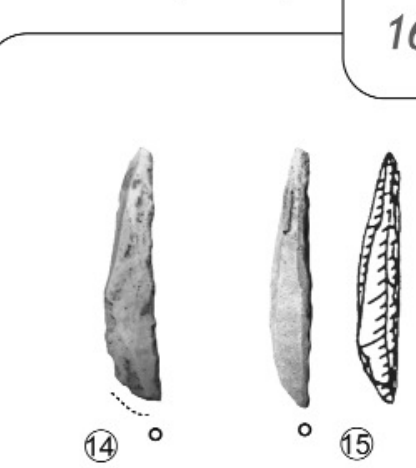

$16.180+/-140 B P(A M S)$

Grotte des Scilles couche $B$

Saint-Germain

La Rivière couches 3 et 4

$16.890+/-130 B P$

C. 4 (AMS)

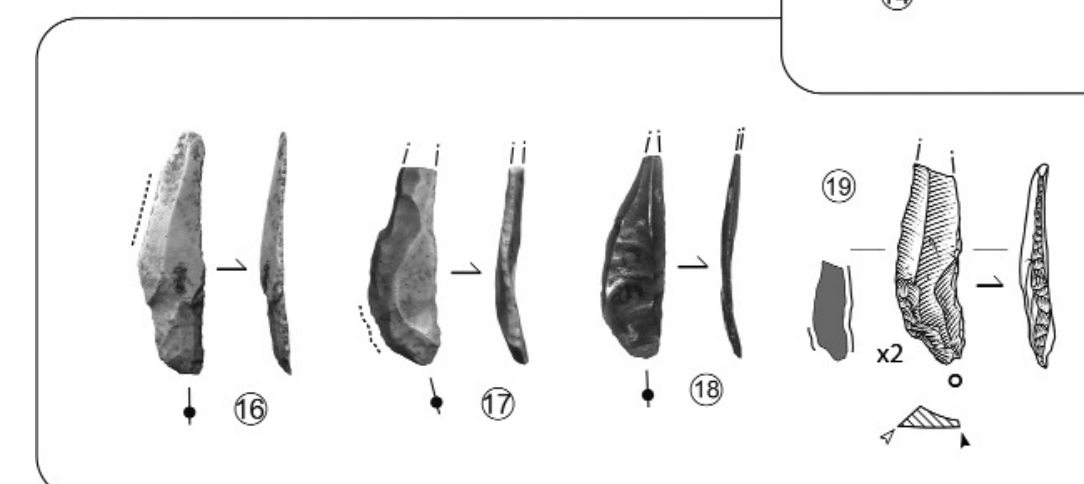

C.4 (AMS)

Grotte de Pégourié

$16.890+/-300$ BP (conv)

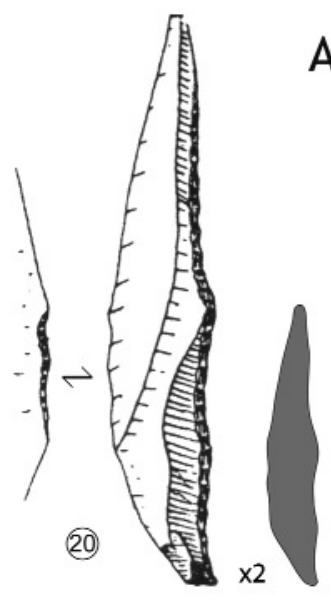

Abri André Ragout couche A-BB'

Figure 31 - Exemples de lamelles à dos dextre marginal provenant de divers gisements situés entre Pyrénées, Quercy, Périgord et Charente (d'après Balout 1958, Lenoir et al. 1991, Sackett 1999, Glory 2008 et Pétillon et al. 2008). Dessin de la pièce $n^{\circ} 21$ S. Ducasse, clichés S. Ducasse et M. Langlais.

Figure 31 - Examples of bladelets with right-hand and marginal back from sites located between Pyrenees, Quercy, Perigord and Charente (after Balout 1958, Lenoir et al. 1991, Sackett 1999, Glory 2008 and Pétillon et al. 2008). Drawing of $n^{\circ} 21$ S. Ducasse, pictures S. Ducasse and M. Langlais. 


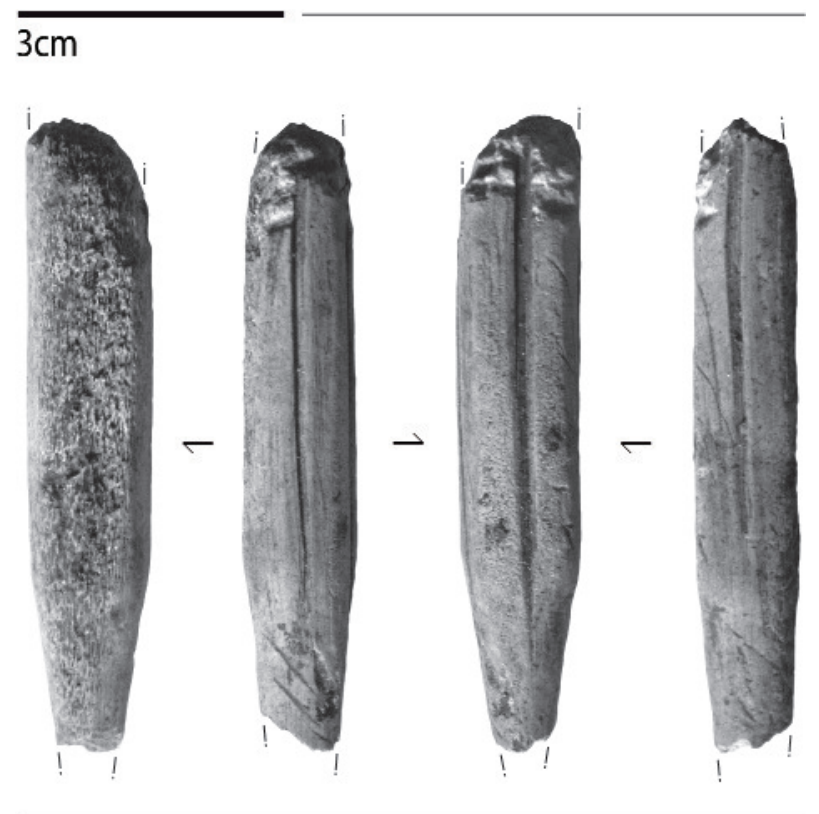

Figure 32 - Pointe de sagaie à trois rainures issue de la série dite de la "Cave à Endives » (Raux et Piel Desruisseaux 1997, fig. 2, $n^{\circ} 50$ ). Cliché S. Ducasse.

Figure 32 - Projectile point with 3 grooves from « Cave à Endives » (Raux et Piel Desruisseaux 1997, fig. 2, $n^{\circ}$ 50). Picture S. Ducasse.

réévaluation simultanée de quelques séries badegouliennes lotoises à fortes composantes lamellaires (Ducasse 2010) ainsi que certains aspects ténus mais originaux de l'exploitation du bois de renne au Petit Cloup Barrat, participent d'une discussion très ouverte, tant sur les variantes - encore peu connues - du Magdalénien inférieur que sur la nature et les rythmes des changements perçus entre ces deux entités.

\section{Un contre-argumentaire ?}

En dehors des incisives sciées qui actuellement n'offrent pas d'équivalent en contexte Magdalénien inférieur, s'il est un élément discordant à même de nourrir une telle discussion, c'est bien l'existence de déchets de débitage du bois de renne par percussion (Chauvière 2009). La vingtaine d'éclats décomptés, formant sur le site de véritables amas, pourrait ne correspondre qu'à une seule et même action technique dont il reste difficile d'établir l'objectif en l'absence de produits finis compatibles. Ce qui pourrait en première lecture être interprété comme une " marque » badegoulienne techno-complexe où le bois de renne semble être exclusivement traité par percussion (Pétillon et Averbouh à paraître, Pétillon et Averbouh à paraître, Pétillon et Ducasse en préparation) - ouvre à notre sens plusieurs voies interprétatives. Gardons d'abord à l'esprit que ce geste technique (la percussion lancée) peut être employé à des fins multiples et que, même si les vestiges que nous avons identifiés au Cloup Barrat sont tout à fait comparables aux exemplaires présents dans les séries badegouliennes du Cuzoul de Vers (Pétillon com. pers.), ces indices sont actuellement trop isolés pour définir un véritable schéma opératoire. En outre, l'état des connaissances sur cet aspect des industries osseuses ne permet pas véritablement d'affirmer que le seul critère de présence/absence de cette " manière de faire " au sein des assemblages revêt une signification culturelle : incontestable au Badegoulien puisque « structurant » (Pétillon com. pers.), rien n'interdit de penser que le recours à la percussion n'était pas, sous des formes et selon des objectifs variés, une composante du Paléolithique supérieur.

Devant le nombre finalement très réduit d'industries osseuses attribuables au Magdalénien inférieur en France, le cas du Petit Cloup Barrat est donc difficile à interpréter : s'agit-il d'une réelle originalité, ou bien les modalités de récolte et d'enregistrement du matériel, quasi-optimales, sont-elles « responsables » de cette mixité technique, invisible à travers le corpus de certains gisements fouillés anciennement et dont le matériel a pu être trié ? Enfin, si tant est que cet équilibre technologique ne témoigne pas de variations économiques (complémentarité synchronique) ou chronologiques (modifications graduelles des industries osseuses, à l'image de ce que l'on peut observer dans le registre lithique : Ducasse 2010), il ne faut également pas négliger l'éventualité - a priori peu probable - d'une contamination stratigraphique puisqu'une « nappe » de raclettes, située dans le fond de l'abri, signe la présence de Badegoulien sur le site (cf. infra). En attendant les résultats d'une série de datations directes sur chacun de ces objets (déchets de rainurage, éclats de percussion) prévue dans le cadre du programme ARTEMIS (programmation 2012), il restera délicat de statuer définitivement sur cette cohabitation technologique.

Un autre élément doit cependant être versé au " dossier » de ce contre-argumentaire : l'analyse de plusieurs séries badegouliennes, appuyée par une recherche bibliographique, nous incite à rester prudents sur l'exclusivité de l'équivalence " lamelles à dos dextre marginal = Magdalénien inférieur ». En effet, qu'il s'agisse d'industries régionales ou non, la présence de ce morphotype lamellaire y est soit formellement attestée (couche 8 de la grotte de Pégourié : fig. $31, n^{\circ} 16$ à 19), soit fortement suspectée (couche 7 de l'abri des Peyrugues ; couche CDE du Piage ; couche A-BB' de l'abri André Ragout : fig. 31, n²0 à 23 ; couche $3 A$ de Solvieux : fig. 31, $\mathrm{n}^{\circ} 24$ à 26). Cette association peut, là aussi, trouver plusieurs explications et avoir, de fait, des implications variées. Si le cas du Piage ne nous sera ici d'aucune utilité puisque lié à une industrie mélangée (Solutréen supérieur et Badegoulien à raclettes au moins), celui de Pégourié c.8 devra, à court terme, faire l'objet d'une attention toute particulière dans le cadre d'une analyse archéostratigraphique ; le signalement de déchets de débitage du bois de renne par double rainurage renforçant par ailleurs 
l'originalité de la série (Séronie-Vivien 1995) ${ }^{25}$. En postulant qu'il ne s'agit pas de mélanges stratigraphiques (hypothèse qui devra prochainement être testée), la réalité de cette association typo-technologique pourrait soit témoigner du continuum techno-économique perçu entre Badegoulien récent et Magdalénien inférieur, soit révéler la limite des arguments d'attribution chrono-culturels proposés plus haut, la présence ou, au contraire, l'absence de raclettes pouvant selon certains auteurs résulter d'un biais de l'échantillonnage voire d'une variation fonctionnelle des occupations (Bodu et al. 2007).

Apports d'une vision élargie à la compréhension de l'assemblage de la couche 4

En l'état actuel des recherches au Petit Cloup Barrat et en dépit de la faible extension des zones fouillées, cette dernière proposition (biais de l'échantillonnage ou variation fonctionnelle) nous semble difficile à soutenir. Au contraire, certains éléments nous amènent à maintenir notre première proposition d'attribution de la couche 4 au Magdalénien inférieur (Chauvière et al. 2008). Ainsi, en dehors de la présence de raclettes au sein des déblais présents dans la zone avant (cf. supra, note 13), leur découverte dans le secteur du fond aurait dans cette perspective pu alimenter une réflexion sur la spatialisation des activités réalisées in situ. Cependant, l'analyse archéo-stratigraphique ${ }^{26}$ permet de privilégier l'hypothèse d'une véritable succession chrono-culturelle : aux pointes à cran solutréennes succèdent les raclettes, ces dernières, présentes à la base de la couche $8 \mathrm{c}$, étant surmontées par un ensemble à lamelles à dos dextre marginal similaire à celui de la couche 4 (fig. 33, cf. supra). Si cette hypothèse nécessite à présent un élargissement de la fouille du fond de l'abri, la série de dates obtenue vient néanmoins la conforter (tabl. 16 et fig. 34). Alors que la mesure de $19.240+/-150$ BP (soit 23.429-22.454 cal. BP) dans le niveau à pointes à cran correspond à un Solutréen supérieur « finissant ", la couche $8 \mathrm{a} 1$, en contact avec la base de la couche $8 \mathrm{c}$ a donné une date de 18.598 +/- 150 BP (soit 22.49321.576 cal. BP) compatible avec les données radiochronologiques AMS disponibles pour le Badegoulien récent quercinois (Clottes et al. dir. à paraître) ou extrarégional (Primault et al. 2007). Un os sélectionné dans la partie supérieure de la couche $8 \mathrm{c}$, en association avec les lamelles à dos dextre marginal, à quant à lui livré une datation qui, cohérente avec la nature de l'assemblage, tend à confirmer son attribution au Magdalénien inférieur : la date de 15.870 +/- 150 BP (19.390-18.813 cal. BP) trouve en effet un équivalent convaincant aux Scilles (couche B : $16.180+/$ - 140 BP, soit 19.555-19.030 cal. BP : Langlais et al. 2010).
Bouclant la boucle, l'obtention récente (Juillet 2011) de deux nouvelles mesures $C^{14}$ pour la couche 4 , tout en affirmant la diachronie de ces occupations (de 17.000 à 16.000 BP, soit 20.000-19.000 cal. BP), permet de renforcer l'interprétation culturelle proposée à partir de nos analyses (tabl. 16). La date la plus ancienne (c.4 base : 16.950 +/- 90 BP, soit 20.388-16.619 cal. BP), parfait écho d'une mesure réalisée sur du matériel non attribué provenant de la couche 9 sous-jacente $(17.000+/-120$, soit 20.494-19.602 cal. BP), place clairement les industries de la couche 4 dans une chronologie postérieure aux dernières expressions badegouliennes (fig. 34).

Soulignons, pour finir, que cette archéo-séquence prend d'autant plus de sens à l'échelle extra-régionale que certaines découvertes - passées et actuelles - s'en font le relai direct. En Périgord, les travaux menés par A. Cheynier à l'abri Lachaud (Cheynier 1953 ; Cretin 2000) sont d'un intérêt tout particulier puisqu'il y décrit une séquence comparable à celle du Petit Cloup Barrat. Se développe, au dessus d'un " niveau très évolué du Solutréen Final » (Cheynier 1953, p. 25), une suite de couches qu'il attribue à différentes phases de son «Proto-Magdalénien »: au «Proto-Magdalénien I (b et c) » succède un niveau de « Proto-Magdalénien II » marqué par la présence de renne, de cheval et de saïga, antilope également chassée et exploitée à Saint-Germain-la-Rivière c.3/4 (Costamagno 1999) et dont un reste - peut-être importé à des fins techniques - a pu être reconnu aux Scilles (matrice d'aiguilles ? Langlais et al. 2010). Du point de vue des équipements lithiques, le fouilleur observe une variation diachronique basée sur la disparition des raclettes dans le Proto-Magdalénien II, moment où apparaissent selon lui " les lamelles à bord abattu simples, sans pointe voulue, et sans troncature retouchée "(Cheynier op. cit., p. 31). Séparé des industries à raclettes par un plancher stalagmitique (op. cit., p. 28), ce dit « ProtoMagdalénien II » semble comprendre un corpus lamellaire similaire à celui que nous décrivions plus haut (fig. 35 ) : les lamelles à dos dextre marginal $\left(n^{\circ} 1\right.$ à 9$)$, dominantes dans la publication, cohabitent avec des exemplaires microlamellaires $\left(n^{\circ} 10\right)$ ainsi qu'avec des armatures à dos envahissant très étroites ( $n^{\circ} 11$ et 12). De fait, et malgré de probables problèmes taphonomiques (op. cit. et $\mathrm{C}$. Cretin com. pers.), cette séquence classique, à industrie osseuse et faune conservée, mériterait une réévaluation globale et interdisciplinaire afin de préciser la pertinence de ces indices.

En Poitou-Charentes, la fouille du Taillis des Coteaux menée par J. Primault tient depuis plusieurs années un rôle essentiel dans le cadre de ces problématiques. Ainsi, les données les plus récentes (Primault et al. 2010) alimentent indiscutablement les propos développés ici : dans le

(25) Notons que la plupart des autres cas de rainurage évoqués en contexte badegoulien font soit l'objet de discussion (Le Cuzoul de Vers où un seul déchet, par ailleurs très ambigu, ne peut actuellement être considéré comme un argument suffisant pour définir un schéma opératoire de débitage du bois de renne par double rainurage : Pétillon et Averbouh à paraître), soit ont tout simplement pu être dissociés des occupations badegouliennes par le biais de datations directes (Lassac: Sacchi et al. dir. à paraitre, Pétillon et Ducasse en préparation; Le Rond-du Barry : Delvigne, Lafarge et Remy com. orale).

(26) Préliminaire compte tenu de la faiblesse des zones exploitées dans ce secteur. 
Projection sagittale

(de $x=190$ à $x=300)$
Projection frontale

(de $y=550$ à $y=600)$

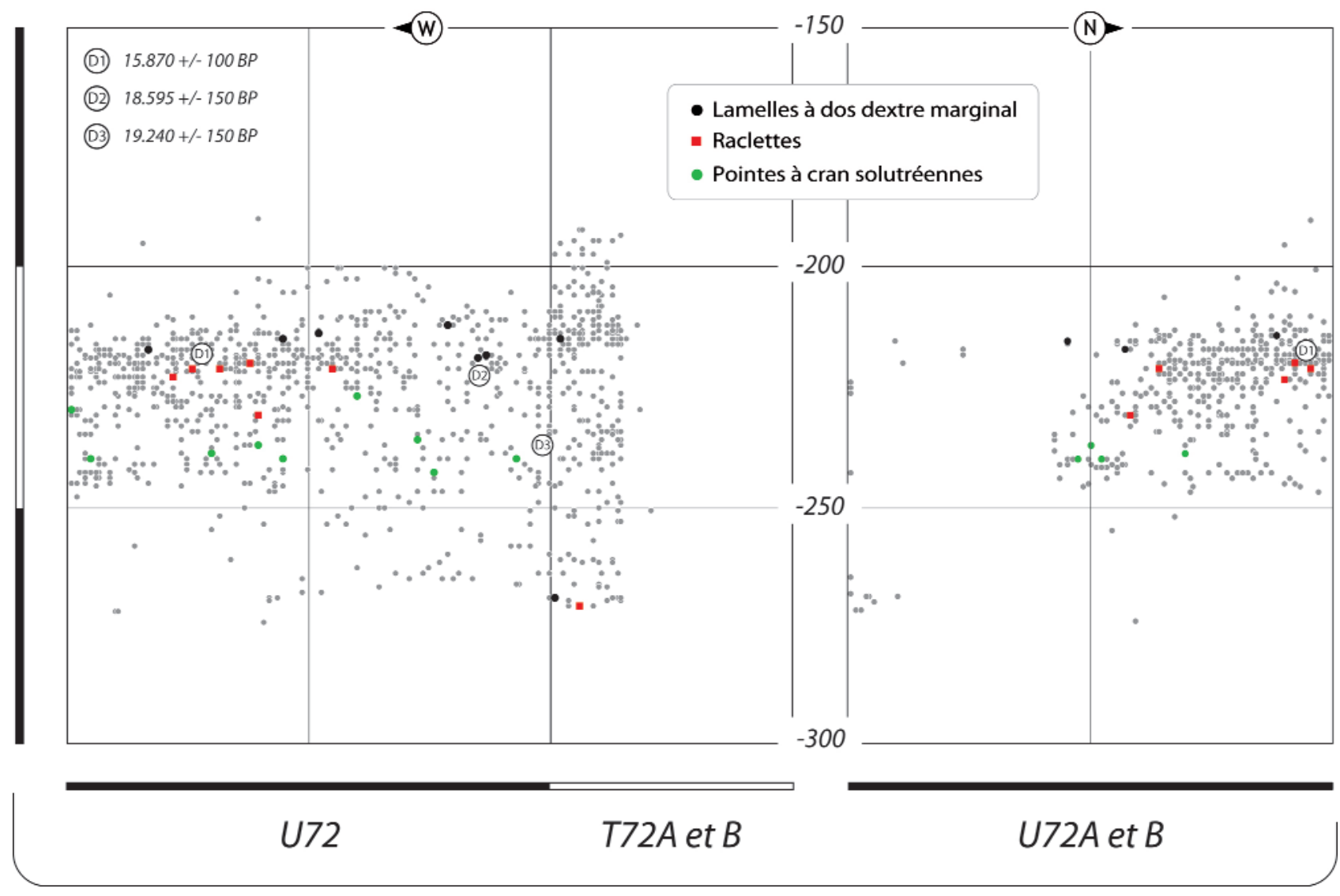

Figure 33 - Une succession Badegoulien récent/Magdalénien inférieur dans le fond de l'abri ? Projection sagittale cumulée des pièces cotées en TU-72 (à gauche) et projection frontale sélective des vestiges issus de U72 (à droite, sous-carrés $A$ et B).

Figure 33 - A succession between recent Badegoulian and Lower Magdalenian inside of the shelter? Cumulated sagittal view of remains fromTU-72 (on the left side) and selected frontal view of remains from U72 (on the right side).

\begin{tabular}{|c|c|c|c|c|c|c|c|c|}
\hline Code échantillon & Couche & Attribution & Matériau & Code laboratoire & Activité 14C & Age BP & Age cal. BP & Validité archéologique \\
\hline PCB04 Q72 n'117 ou 193 & sommet c.4 & Magdalénien inf/moy & Os (OTM) & Lyon-4712 (SacA-9865) & $22,85 \% \pm 0,18$ & $11.860 \pm 60$ & $13.858-13.479$ & mauvaise \\
\hline PCB07 Q71B dec. 13, n'312 & base c. 4 & Magdalénien inférieur & Os (OTM) & Lyon-4834 (SacA-10377) & $26,86 \% \pm 0,19$ & $10.560 \pm 60$ & $12.649-12.227$ & mauvaise \\
\hline PCB03 Q71 dec. $15, n^{\circ} 6$ & c. 9 & à définir & Os (OTM) & Lyon-5605 (SacA-12681) & $12,04 \% \pm 0,18$ & $17.000 \pm 120$ & $20.494-19.602$ & indéterminée \\
\hline PCB04 S70D dec.10, n²42 & sommet c.4 & Magdalénien inférieur & Os (OTM) & Lyon-7831 (SacA-22778) & $13,03 \% \pm 0,14$ & $16.370 \pm 90$ & $19.877-19.315$ & bonne \\
\hline PCB06 S70D dec.31, n¹06 & base c. 4 & Magdalénien inférieur & Os (OTM) & Lyon-7832 (SacA-22779) & $12,13 \% \pm 0,13$ & $16.950 \pm 90$ & 20.388-19619 & bonne \\
\hline PCB05 U72 dec. $19, n^{\circ} 469$ ou 430 & sommet c.8c & Magdalénien inférieur & Os (OTM) & Lyon-5603 (SacA-12679) & $13,87 \% \pm 0,18$ & $15.870 \pm 100$ & $19.390-18.813$ & bonne \\
\hline PCB05 U72 dec.25, n608 & c. $8 \mathrm{al}$ & Badegoulien & Os (OTM) & Lyon-3366 (Poz) & $9,88 \% \pm 0,19$ & $18.595 \pm 150$ & 22.493-21.576 & bonne \\
\hline PCB05 U72 dec.33, n'720 ou 721 & $c .8 \mathrm{a} 2$ & Solutréen supérieur & Os (OTM) & Lyon-5604 (SacA-12680) & $9,11 \% \pm 0,17$ & $19.240 \pm 150$ & 23.429-22.454 & bonne \\
\hline РСВ08 T71C dec. 20 ou $22, n^{\circ} 100$ ou 113 & c.10 & à définir & Os (OTM) & Lyon-5606 (SacA-12682) & $6,53 \% \pm 0,17$ & $21.910 \pm 210$ & $27.010-25.600$ & indéterminée \\
\hline
\end{tabular}

Tableau 16 - Mesures d'âge C14 AMS obtenues au Petit Cloup Barrat (OTM : ongulé de taille moyenne).

Table 16 - AMS datings of Petit Cloup Barrat (OTM : medium size ungulate). 


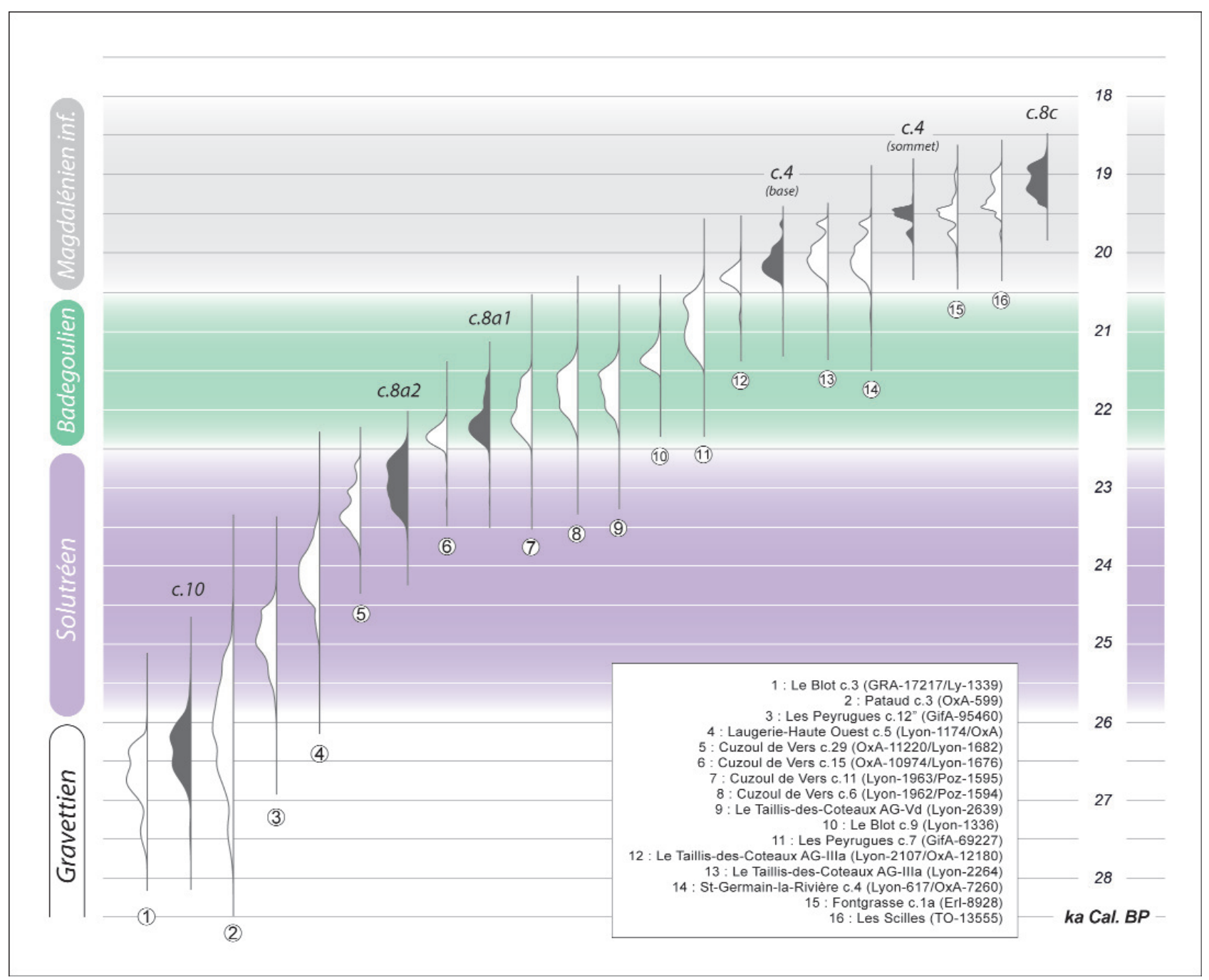

Figure 34 - Diachronie des occupations au Petit Cloup Barrat d'après les dates obtenues au fond de l'abri (U72-T71). Données de comparaison AMS d'après Bazile 2006, Langlais 2007, Primault et al. 2007, Klaric 2008, Pétillon et al. 2008, Renard 2008, Clottes et al. à paraître et (la date de 16.580 +/- 80 BP - GRA 18788/Lyon 1548 - obtenue sur le site de plein air de Lassac n'a pas été prise en compte, son association avec les vestiges badegouliens ayant été récemment mise en cause : Sacchi et al. dir. à paraître; Pétillon et Ducasse en préparation).

Figure 34 - Chronology of the occupations documented at Le Petit Cloup Barrat according to the datings obtained inside the shelter (U72T71). Comparison data after Bazile 2006, Langlais 2007, Primault et al. 2007, Klaric 2008, Pétillon et al. 2008, Renard 2008, Clottes et al. to be published and (the 16.580 +/- 80 BP - GRA 18788/Lyon 1548 - date obtained at the open air site of Lassac has not been considered because of his problematic link with the badegoulian industries: Sacchi et al. dir. to be published; Pétillon et Ducasse forthcoming).
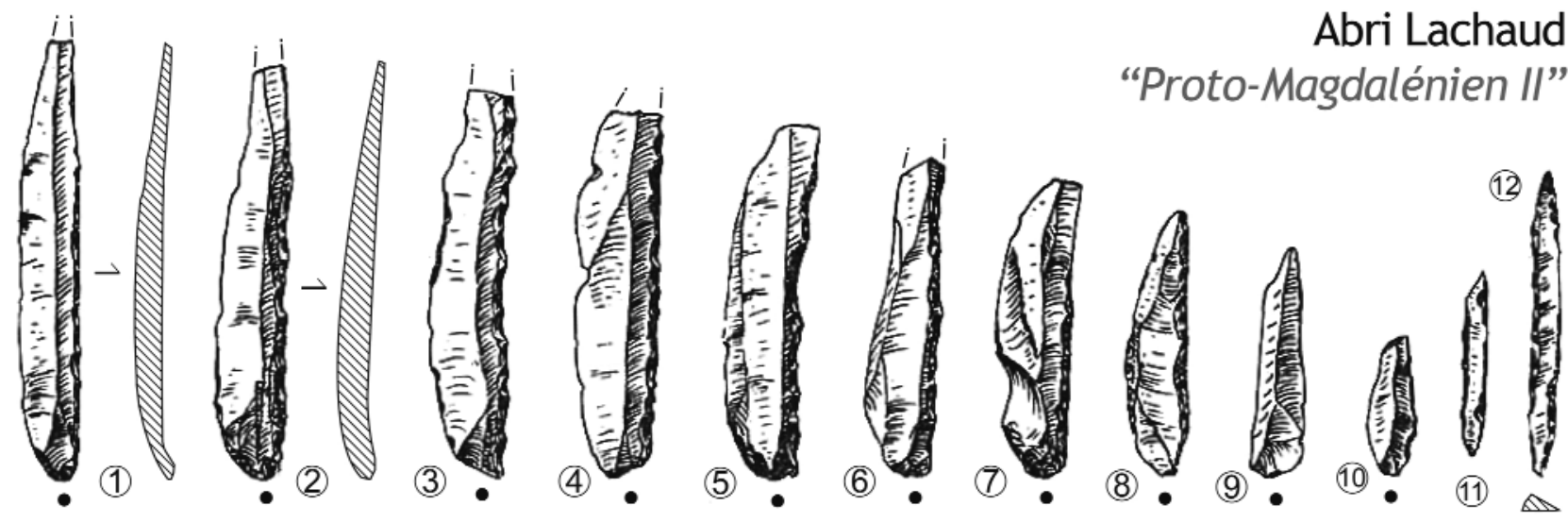

Figure 35 - Lamelles à dos dextre marginal ( $n^{\circ} 1$ à 9), microlamelle à dos ( $\left.n^{\circ} 10\right)$ et lamelles étroites à dos envahissants $\left(n^{\circ} 11\right.$ et 12$)$ du "Proto-Magdalénien II » de l'abri Lachaud (d'après Cheynier 1953, modifié).

Figure 35 - Bladelets with right-hand and marginal back ( $n^{\circ} 1$ to 9), backed microbladelet ( $\left.n^{\circ} 10\right)$ and narrow backed bladelets ( $n^{\circ} 11$ et 12) from the « Proto-Magdalénien II » of Lachaud shelter (after Cheynier 1953, modified). 
secteur de l'avant grotte (AG), entre un niveau Badegoulien récent $(\mathrm{Vd})$ daté à 18.140 +/- $145 \mathrm{BP}$ (soit 22.17021.310 cal. BP) et un niveau Magdalénien inférieur présent aux alentours de 17.500 BP (niveau AG-IIlb) a été découverte une industrie sans raclettes et à "lamelles à retouches marginales ${ }^{27}$ qui, également attribuée au Magdalénien inférieur, présente une industrie osseuse tout aussi caractéristique (Jérôme Primault com. pers. et Annabelle Gioé, Master 1 en cours).

\section{Vers une perspective interrégionale}

Quelles que soient les questions encore posées sur l'industrie de la couche 4 du Petit Cloup Barrat notamment celles relatives au lien qu'elle entretient avec l'entité badegoulienne telle qu'elle se définit actuellement (Bodu et al. dir. 2007) - se fait jour à travers elle une problématique plus large intégrant différents registres d'activité. Répondant aux cortèges lithologiques des couches 3 et 4 de Saint-Germain-La Rivière, de Gandil (Langlais 2010) ou de la couche B des Scilles (Pétillon et al. 2008 ; Langlais et al. 2010a), les premières observations petro-archéologiques réalisées sur la couche 4 semblent entériner les relations déjà entrevues par le biais des équipements techniques entre Pré-Pyrénées, Quercy, Périgord et Poitou-Charentes (fig. 36 et 37). L'apport probable de silex dit du « Grain de Mil » ainsi que l'ébauche d'anneau ouvert qui, comme nous le disions, confirme les relations Charente/Quercy tout en précisant, pour la première fois, l'attribution chrono-culturelle de ce type d'objet (cf. supra) ${ }^{28}$, se retrouve indirectement associé aux fameuses lampes en grès connues à Lascaux, aux Scilles, mais aussi au Bois-du-Roc, gisement voisin de la grotte du Placard et de l'abri André Ragout (ce dernier ayant, pour rappel, livré un assemblage à lamelles à dos dextre marginal : Ducasse 2010 et fig. 31). II semble ainsi que l'on puisse progressivement tisser des liens à grande distance à partir de la diffusion d'objets très particuliers. Ces liens signent vraisemblablement un vaste réseau d'interactions sociales au cœur du DMG, réseau au sein duquel le Quercy s'inscrit pleinement. À ce titre, la poursuite et l'élargissement ${ }^{29}$ des fouilles au Petit Cloup Barrat est pleine de promesses, à même de nourrir considérablement cette problématique.

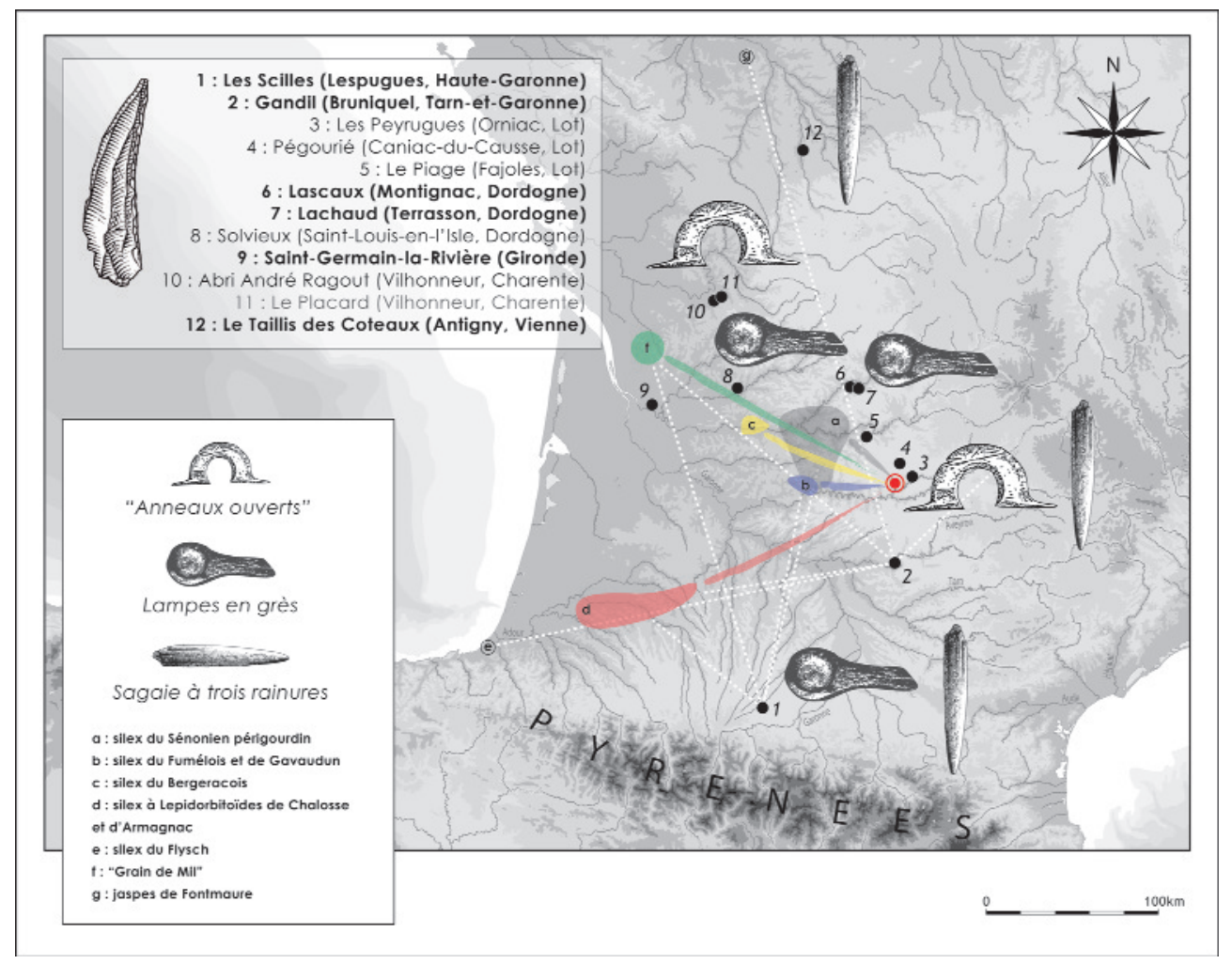

Figure 36 - Des contreforts des Pyrénées au Poitou, le Petit Cloup Barrat au cœur d'un "réseau culturel »? Correspondances entre différentes industries à lamelles à dos dextre marginal (le Placard mis à part) abritant conjointement pointe de sagaie à trois rainures et/ou anneaux ouvert en bois de renne et/ou lampes en grès (cercle rouge : Cloup Barrat).

Figure 36 - From the foothills of the Pyrenees to Poitou, Le Petit Cloup Barrat in the heart of a "cultural network »? Correspondences between the different industries containing bladelets with right-hand and marginal back (excepted Le Placard) and possessing projectile point with 3 grooves and/or reindeer antler opened ring and/or sandstone lamps (red circle : Cloup Barrat).

(27) Nous remercions vivement Jérôme Primault et Annabelle Gioé (Master 1 à l'Université de Toulouse 2) pour la journée que deux d'entre nous (ML et SD) ont pu passer devant ce matériel.

(28) Seule ébauche connue à ce jour, elle pourrait d'ailleurs plus facilement se prêter à un prélèvement pour datation C14.

(29) L'extension potentielle de la couche 4 dans la doline peut être évaluée à près de $50 \mathrm{~m}^{2}$ 


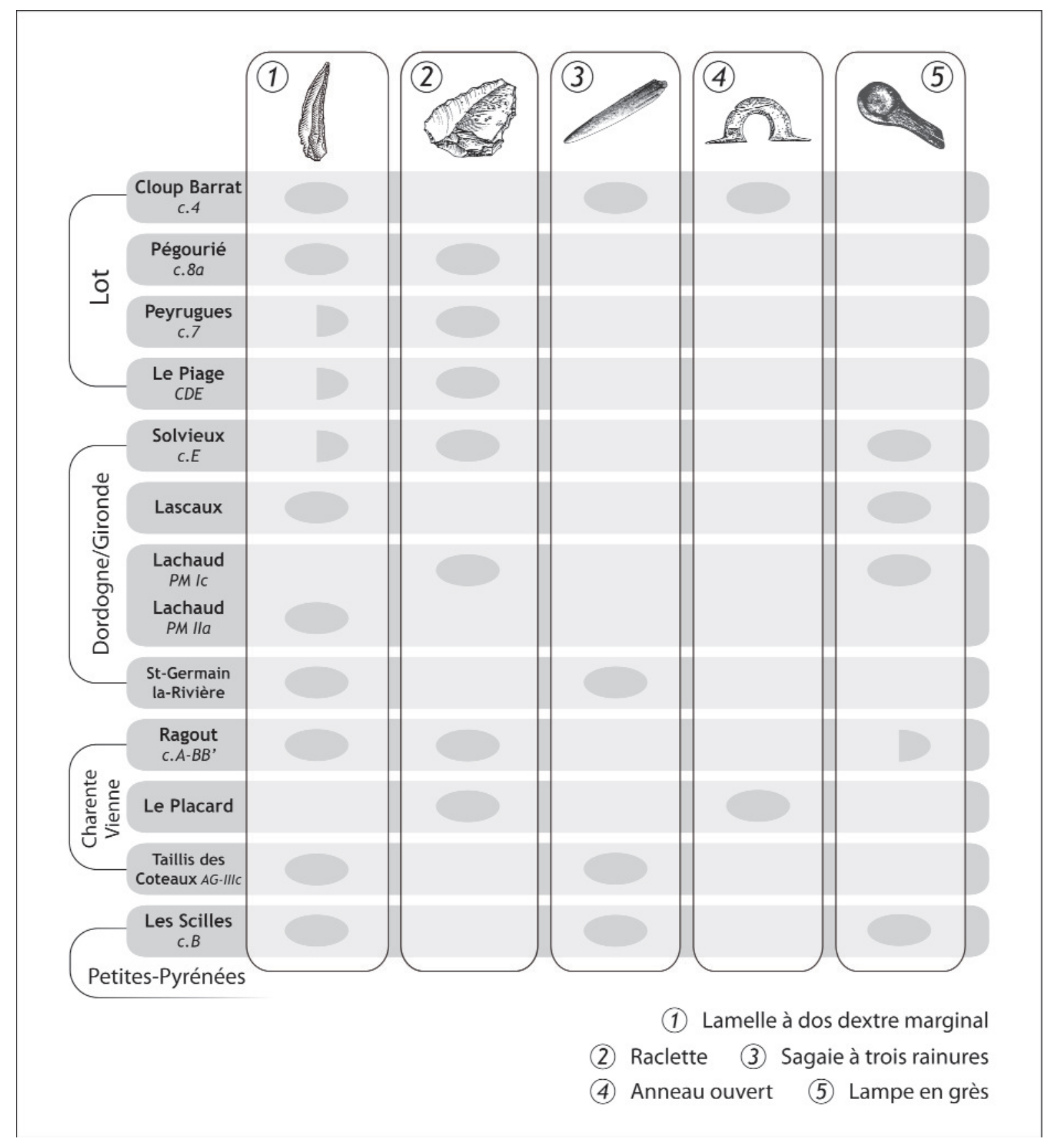

Figure 37- Récapitulatif des données utilisées afin d'établir la figure 36 (demi-ovale : présence probable et/ou à vérifier). La lampe signalée avec réserve à l'abri André Ragout correspond à l'exemplaire attribué sans plus de précision au "Bois du Roc ». Document sujet à variations.

Figure 37 - Summary of data used to established figure 36 (half-oval: probable presence and/or to verify). Note that the sandstone lamp linked with caution to the André Ragout rock shelter is generally attributed with no more precision to the «Bois du Roc ». Document may vary in the future. 


\section{Remerciements}

Cette publication a été réalisée dans le cadre d'un programme d'APP grâce au support du Service Régional de l'Archéologie de Midi-Pyrénées que nous remercions vivement. Toute notre gratitude va également à Françoise Echasseriaud et, à travers elle, à l'association "Archéologies ». Nous tenons à remercier Bertrand Defois qui, chaque année, nous permet un accès facilité aux salles d'études du Musée de Pech Merle, ainsi que Pascal Gaudebert, propriétaire du site du Petit Cloup Barrat et Paul Bouscary, propriétaire du Malpas. Toute notre reconnaissance à John Hollier qui a assuré la traduction anglaise de la version abrégée de l'article. Un grand merci également à Jean-Marc Pétillon pour ses avis, conseils et remarques sur l'industrie osseuse du Petit Cloup Barrat ainsi qu'à Claire Houmard pour les informations relatives à l'industrie osseuse du Magdalénien inférieur du Taillis des Coteaux. Merci à Catherine Cretin et à Christophe Fourloubey pour leurs relectures efficaces, enrichissantes et enthousiastes. Que l'ensemble de l'équipe scientifique - passée et actuelle - soit ici remerciée : Lucile Beck, Catherine Cretin, Loïc Daulny, Jérémie Ferrand, Dominique Henri-Gambier, Mathieu Luret, Xavier L'Homme, Michel Menu, Xavier Muth, Julien Oppliger, Patrick Paillet, Jacqueline Studer, Caroline Renard, Olivier Randon et Philippe Wagneur. Nous n'oublions pas l'ensemble des fouilleurs qui se sont succédé au Cloup Barrat depuis 2004, et plus particulièrement les plus assidus d'entre eux : Olivier Randon, Ophélie Bonifas, Justine Bourriot, Jérémie Ferrand, Claire Fradet, Tiphaine le Diouron. Enfin, nous adressons un remerciement spécial à Valérie Castel pour son engagement de tous les instants et son aide plus que précieuse.

\section{Références bibliographiques}

ALLAIN J. 1979 - L'industrie lithique et osseuse de Lascaux. In : Arl. Leroi-Gourhan et J. Allain dir., Lascaux inconnu, CNRS Editions, Gallia Préhistoire, suppl. 13, p. $87-120$.

ALLAIN J., FRITSCH R., RIGAUD A., TROTIGNON F. 1974 Le débitage du bois de renne dans les niveaux à raclettes du Badegoulien de l'abri Fritsch et sa signification. In: $\mathrm{H}$. Camps-Fabrer, (dir.), Premier colloque international sur l'industrie de l'os dans la Préhistoire, Abbaye de Sénanque, avril 1974, Aix-en-Provence, Editions de I'Université de Provence, p. 67-74.

ALLARD M. 1992 - Les Magdaléniens de l'abri des Peyrugues à Orniac (Lot). Leur culture et leurs relations avec le milieu. In : Le peuplement magdalénien. Paléographie physique et humaine, CTHS, Paris, p. 377-385, 4 fig.

ALLARD M., ALIX Ph. et JUILLARD F. 1995 - Strategie de fouilles et principaux résultats dans l'abri paléolithique des Peyrugues. Préhistoire quercynoise, 2, p. 2-13.
BALOUT L. 1958 - L'abri Andre Ragout au Bois-du-Roc (Vilhonneur, Charente). Bulletin de la Société Préhistorique Française, 55(9), p. 589-627.

BAZILE F. 2006 - Datations du site de Fontgrasse (VersPont-Du-Gard, Gard.). Implications sur la phase ancienne du Magdalénien en France Méditerranéenne, Bulletin de la Société Préhistorique Française, 103(3), p. 597-602.

BERGANZA E. et ARRIBAS J.-L. 2010 - Dientes de herbívoros serrados e incisos de la cueva de Santa Catalina (Lekeitio, Bizkaia), Munibe, 61, p. 57-70.

BINFORD L. R. 1981 - Bones. Ancient Men and modern Myths, New York, Academic Press.

BODU P., CHEHMANA L., CRETIN, C., DUCASSE S. et LANGLAIS M. dir. 2007 - Le dernier Maximum Glaciaire et après... en France et en Espagne. Synthèses régionales et réflexions autour de la diversité des cultures matérielles de 19000 à 14000 BP. Actes de la Table Ronde/Séance de la SPF de Toulouse, 9 décembre 2006, Bulletin de la Société Préhistorique Française, 100(4), 169 p.

BODU P., CHEHMANA L. et DEBOUT G. 2007 - Le Badegoulien de la moitie nord de la France. Un état des connaissances, Bulletin de la Société Préhistorique Française, 104(4), p. 661-680.

BON F. 2002 - L'Aurignacien entre mer et océan. Réflexion sur l'unité des phases anciennes de l'Aurignacien dans le Sud de la France. Mémoire de la Société Préhistorique Française, 29, $253 \mathrm{p}$.

BON F., DARTIGUEPEYROU S., GARDERE Ph. et MENSAN R. 1996 - Caractérisation du silex de Chalosse. Antiquités Nationales, 28, p. 33-38.

BONNISSENT D., CHAUVIERE F.-X. 1999 - L'industrie sur matières dures animales. In : L'habitat magdalénien de la grotte du Bourrouilla à Arancou (Pyrénées-Atlantiques), Gallia préhistoire, 41, p. 36-53.

BOUCHUD J. 1966 - Essai sur le renne et la climatologie du Paléolithique moyen et supérieur, Périgueux, Magne, 300 p., 55 fig., XIII pl., LXXI tab.

BOURDIER C., CHEHMANA L., PÉTILLON J.-M. et VALLADAS H., sous presse - L'abri-sous-roche orné de Reverdit (Sergeac, Dordogne) : l'apport d'une approche pluridisciplinaire à l'élaboration d'un nouveau cadre chronoculturel. In : CRETIN C., CASTEL J.-C., FERULLO O. (org.) Deuxième moitié et fin du Paléolithique supérieur. Pour une confrontation entre le modèle classique et les perceptions interdisciplinaires actuelles sur le thème des unités, continuités et discontinuités), XXVIle Congrès Préhistorique de France, Bordeaux - Les Eyzies, 31 mai 5 juin 2010, Session F.

BRACCO J.P., MORALA A., CAZALS N., CRETIN C. et FERRULLO O. 2003 - Peut-on parler de débitage discoïde au Magdalénien ancien/Badegoulien ? Présentation d'un schéma opératoire de production d'éclats courts 
normalisés. In : M. PERESANI (éd.), Discoid lithic technology Advances and implications, BAR 1120, p. 83-115.

CAMUS H. 2009 - Synthèse géologique et géomorphologique du site du Petit Cloup Barrat. In : Castel J.-C., Chauvière F.-X., Randon O., Camus, Ducasse S., Ferrand J., Langlais M., Morala A., Muth X., Renard C. et Turq A. - Petit Cloup Barrat, Cabrerets, Lot (46), rapport de fouille programmée deuxième triennale 2006-2008. Rapport d'évaluation à l'intention des services administratifs du Ministère de la Culture, p.113-168. http://le-petit-cloup-barrat.fr ; rubrique « Rapports »

CASTEL J.-C. 1999 - Comportements de subsistance au Solutréen et au Badegoulien d'après les faunes de Combe Saunière (Dordogne) et du Cuzoul de Vers (Lot). Thèse de I'Université Bordeaux I, non publiée.

CASTEL J.-C. 2003 - Économie de chasse et d'exploitation de l'animal au Cuzoul de Vers (Lot) au Solutréen et Badegoulien, Bulletin de la Société Préhistorique Française, 100(1), p. 41-66.

http://www.persee.fr/web/revues/home/prescript/article/b spf_0249-7638_2003_num_100_1_12792

CASTEL J.-C. 2005 - Economie préhistorique, boucherie et décompte des fragments osseux. Revue de Paléobiologie, Genève, vol. spéc. 10, Hommages à Louis Chaix. p. 23-30.

CASTEL J.-C. 2010 - Comportements de subsistance au solutreen et au badegoulien d'apres les faunes de combe saunière (Dordogne) et du Cuzoul de Vers (lot), Editions Universitaires Européennes, Sarrebrück, 635 p.

CASTEL J.-C., CHAUVIÈRE F.-X., L'HOMME X., BERTRAN P., DAULNY L., DEFOIS B., DUCASSE S., LANGLAIS M., MANCEL D., MORALA A., RENARD C., TURQ A. 2005 - Le Petit Cloup Barrat (Cabrerets, Lot) : un nouveau site du Paléolithique supérieur récent sur les plateaux du Quercy. Bulletin de la Préhistoire du Sud-ouest, t. 12-1, p.91-92. http://www.persee.fr/web/revues/home/prescript/article/b spf_0249-7638_2006_num_103_2_13432

CASTEL J.-C., CHAUVIÈRE F.-X., L'HOMME X., CAMUS H. 2006 - Un nouveau site du Paléolithique supérieur récent : le Petit Cloup Barrat (Cabrerets, Lot, France). Bulletin de la Société Préhistorique Française, 103(2), p. 263-273.

CASTEL J.-C., COUMONT M.-P., BRUGAL J.-P., LAROULANDIE, V., CAMUS, H., CHAUVIĖRE, F.-X., COCHARD D., GUADELLI J.-L., KUNTZ, D., MARTIN H., MOURRE V. 2008 - La fin du Paléolithique supérieur en Quercy : l'apport de l'Igue du Gral (Sauliac-sur-Célé, Lot). In : Jaubert J., Ortega I. et Bordes J.-G. : Les sociétés du Paléolithique dans un Grand Sud-Ouest : nouveaux gisements, nouveaux résultats, nouvelles méthodes. Journées de la Société Préhistorique Française, Bordeaux, 24-25 novembre 2006, Société Préhistorique Française, Mémoire XLVII, p. 335-353.
CASTEL J.-C., KUNTZ D., CHAUVIERE F.-X., avec la collaboration de GERBE M., et JUILLARD F., à paraître L'exploitation du monde animal au Paléolithique supérieur en Quercy, In : Modalités d'occupations et exploitation des milieux au Paléolithique dans le Sud-Ouest de la France: l'exemple du Quercy, Actes du colloque 67 du XVeme congres de I'UISPP, Lisbonne, Jarry M., Brugal J.-Ph. et Ferrier C. dir., Paleo.

CASTEL J.-C., CHAUVIERE F.-X., à paraître - Du Pléniglaciaire au Tardiglaciaire en Quercy central : continuités, discontinuités dans l'exploitation du monde animal, In : CRETIN C., CASTEL J.-C., FERULLO O. (org.) Deuxième moitié et fin du Paléolithique supérieur. Pour une confrontation entre le modèle classique et les perceptions interdisciplinaires actuelles sur le thème des unités, continuités et discontinuités), XXVIle Congrès Préhistorique de France, Bordeaux - Les Eyzies, 31 mai 5 juin 2010, Session F.

CAUX S. 2011 - Le silex dit « grain de mil ». Bilan de sa caractérisation géologique ; traitement archéologique. L'exemple de la séquence aurignacienne de la Roche-àPierrot (Saint-Césaire, Charente-Maritime). Mémoire de Master 2 de l'Université de Bordeaux 1, 55 p.

CAZALS N. 2000 - Constantes et variations des traits techniques et économiques entre le Magdalénien « inférieur » et « moyen» : analyse des productions lithiques du Nord de la péninsule ibérique. Thèse de Doctorat, Université de Paris I Panthéon-Sorbonne, ex. multigraphié.

CAZALS N. et LANGLAIS M. 2006 - La place d'Ekain (couche VII) au sein du Magdalénien basco-cantabrique : nouvelles contributions sur l'organisation des productions lithiques. In : Homenaje a Jesus Altuna, Munibe 57, p. 117-191.

CHALARD P., DUCASSE S., BON F., BRUXELLES L., TEYSSANDIER N., RENARD C., GARDERE P., GUILLERMIN P., LACOMBE S., LANGLAIS M., MENSAN R., NORMAND CH., SIMONNET R. et TARRINO A. 2010 Diffusion et exploitation d'un traceur lithologique au cours du Paléolithique supérieur dans le sud de la France : l'exemple du type Chalosse. In : Quarries : where it all began, A. Burke dir., Actes du congrès international de la SAA, Porto-Rico 2006, p. 13-22.

CHAUVIERE F.-X. 2003 - Quand le « rebut « vaut le « bel objet «: pour une approche technique des industries néolithiques sur os et sur dentine de Suisse occidentale. Préhistoire et Anthropologie Méditéranéenne, 12, p. 129135, 3 fig.

CHAUVIERE F.-X. 2006 - Fonds commun et originalité du matériel dentaire travaillé dans le Magdalénien d'Arancou (Pyrénées-Atlantiques), In: C. Chauchat (Ed.), Préhistoire du Bassin de l'Adour : bilans et perspectives, Colloque Izpegi, CNRS, Saint-Etienne de Baigorry (France), 19 janvier 2002, Saint-Etienne-de-Baïgorry, Edition Izpegi de Navarre, p. 225-248. 
CHAUVIERE F.-X. 2009 - Industries et parures sur matières dures d'origine animale du Petit Cloup Barrat : Bilan 20062008, In : J.-C. CASTEL, Petit Cloup Barrat, Cabrerets, Lot. Rapport de fouille programmée, $2^{\text {ème }}$ triennale 2006-2008, p. 227-244.

http://le-petit-cloup-barrat.fr ; rubrique « Rapports »

CHAUVIERE, F.-X., RIGAUD A. 2005 - Les « sagaies « à base raccourcie ou les avatars de la typologie : du technique au " non-fonctionnel « dans le Magdalénien à navettes de la Garenne, In : V. Dujardin, (Dir.), Table ronde sur le Paléolithique supérieur récent : Industrie osseuse et parures du Solutréen au Magdalénien en Europe, Angoulême, 28-30 mars 2003. Mémoire, XXXIX, Paris, Société Préhistorique Française, p. 233-242, 12 fig.

CHAUVIERE F.-X., CASTEL J.-C., L'HOMME X., CAMUS, H., LANGLAIS, M., DAULNY L., DEFOIS B., DUCASSE S., MORALA A., RENARD C., TURQ A. 2008 - Ein neuer Fundort aus dem späten Jungpaläolithikum in Südwestfrankreich: Le Petit Cloup Barrat (Cabrerets, Lot). Quartär, 55, 159-163.

CHEHMANA L., HOLZEM N., PELEGRIN $J$ et BAZIN P. 2009 - La fonction des pièces de la Bertonne : un problème en partie résolu, Paleo 21, p. 65-102.

CHEYNIER A. 1953 - Stratigraphie de l'abri Lachaud et les cultures des bords abattus, Archivo de Prehistoria Levantina, 4, p. 25-55, 20 fig.

CHEYNIER A. 1956 - La Bombetterie, station aurignacienne de plein air à Cublac (Corrèze). In : Libro homenaje al conde de la Vega del Sella, Diputación provincial de Asturias, SIA, p. 95-106.

CHRISTENSEN M. et VALENTIN B. 2004 - Armatures de projectiles et outils. In : N. PIGEOT (dir.), Les derniers Magdaléniens d'Etiolles. Perspectives culturelles et paléohistoriques, Gallia Préhistoire, XXVIlème supplément, p. 107-160.

CLOTTES J., DUPORT L., FERUGLIO V. 1990 - Les signes du Placard. Préhistoire Ariégeoise, Bulletin de la Société Préhistorique Ariège-Pyrénées, XLV, p. 15-49, 24 fig.

CLOTTES J., GIRAUD J.-P. et CHALARD P. dir., à paraitre Solutréen et Badegoulien au Cuzoul de Vers. Des chasseurs de rennes en Quercy. E.R.A.U.L.

COLONGE D., CHALARD P., BILOTTE M., DUCASSE S. et PLATEL J.-P. 2011 - Nouvelle découverte d'un gîte à silex à Lepidorbitoides $s p$. dans le Sud-Ouest de la France (Saint-Aubin, Gers) et implications archéologiques. Bulletin de la Société Préhistorique Française, 108(3), p. 561-564.

COSTAMAGNO S. 1999 - Stratégie de chasse et fonction des sites au Magdalénien dans le sud de la France, Thèse de doctorat de l'Université de Bordeaux I, ex. multigraphié, 495 p.

COUMONT, M.-P., CASTEL, J.-C. 2007 - Etude taphonomique d'un aven-piège du Tardiglaciaire, l'Igue du Gral (Sauliac-sur-Célé, Lot). In : Evin J., dir. Un siècle de construction du discours scientifique en préhistoire, Actes du $26^{e}$ Congrès Préhistorique de France, Colloque du centenaire de la S.P.F., Avignon, 21-25 septembre 2004. Société Préhistorique Française, volume3, p.503-514.

CRETIN C. 2000 - Tradition et variabilité dans le comportement technique. Le cas du Badegoulien et du Magdalénien en Périgord. Thèse de Doctorat, Université de Paris I, 2 tomes, $451 \mathrm{p}$.

DAULNY L. 2004 - Vestiges lithiques hors silex du Petit Cloup Barrat (Cabrerets, Lot). Fouille 2004 : analyse préliminaire, Rapport d'étude, In : Castel, J.-C., Chauvière, F.-X., L'Homme, X., Petit Cloup Barrat, Cabrerets, Lot (46), rapport de fouille programmée 2004. Rapport d'évaluation à l'intention des services administratifs du Ministère de la Culture, p. 93-99, 3 fig. http://le-petit-cloup-barrat.fr ; rubrique « Rapports »

DAULNY L. 2005 - Vestiges lithiques hors silex du Petit Cloup Barrat (Cabrerets, Lot) - Fouille 2005, analyse préliminaire. In : Castel, J.-C., Chauvière, F.-X., L'Homme, X., Camus, H., Daulny, L., Defois, B., Ducasse, S., Langlais, M., Mancel, D., Morala, A., Renard, C., Turq, A. - Petit Cloup Barrat, Cabrerets, Lot (46), rapport de fouille programmée 2005. Rapport d'évaluation à l'intention des services administratifs du Ministère de la Culture. http://le-petit-cloup-barrat.fr ; rubrique « Rapports »

DUCASSE S. 2004 - Produire des lames et des lamelles au Badegoulien, technologie et économie : analyse préliminaire d'ensembles lithiques du Badegoulien méridional. Mémoire de DEA, Université de Toulouse-Le Mirail, $67 \mathrm{p}$.

DUCASSE S. 2010 - La "parenthèse » badegoulienne : fondements et statuts d'une discordance industrielle au travers de l'analyse techno-économique de plusieurs ensembles lithiques méridionaux du Dernier Maximum Glaciaire. Thèse de doctorat, Université de Toulouse-Le Mirail, ex. multigraphié, $460 \mathrm{p}$.

http://halshs.archives-ouvertes.fr/tel-00565784/

DUCASSE S. et LANGLAIS M. 2007 - Entre Badegoulien et Magdalénien inférieur, nos cœurs balancent... Approche critique des industries lithiques du Sud de la France et du nord-est espagnol entre 19.000 et 16.500 BP. Bulletin de la Société Préhistorique Française, 104 (4), p. 771-785. http://www.persee.fr/web/revues/home/prescript/article/b spf_0249-7638_2007_num_104_4_13622

DUCASSE S. et LANGLAIS M. 2008 - Interprétation technologique et discussion autour du statut culturel des « pièces de la Bertonne ». L'exemple de la série lithique de Seyresse (Landes, France), Paleo 20, p. 59-88. http://paleo.revues.org/index1674.html

FERUGLIO V. 1992 - Fiche Baguettes demi-rondes, In: Bâtons percés, baguettes, Fiches typologiques de l'industrie osseuse préhistorique, cahier V Treignes, CEDARC, p. 71-83, 6 fig. 
FERUGLIO V., LEROY-PROST C. 2001 - Fiche objets arciformes (en forme d'oméga), In: D. Ramseyer, (Dir.), Objets méconnus. Industrie de l'os préhistorique, cahier IX, Paris, Editions Société Préhistorique Française, p. 17-21, 2 fig.

FOURLOUBEY C. 1996 - Etude de la variabilité des industries post-solutréennes de transition vers le Magdalénien par l'analyse des travaux de taille du silex. L'exemple du Badegoulien et du Magdalénien ancien de plein air dans la moyenne vallée de l'Isle (Dordogne). Thèse de Doctorat, Université de Bordeaux I, 452 p.

GLORY A. (†) 2008 - Les recherches à Lascaux (19521963). Textes et documents recueillis, présentés et commentés par B. et G. Delluc, Gallia Préhistoire, supplément 39, CNRS, Paris, 199 p.

GRIGGO C. 1997 - La faune magdalénienne de l'abri Gandil, Bruniquel (Tarn-et-Garonne) : études paléontologique, taphonomique et archéozoologique, Paléo 9, p. 279-294.

JUILLARD F. 2009 - La macro-faune de l'abri des Peyrugues : biométrie et odontométrie. Préhistoire du Sud-Ouest 17 (2), 151-177.

KLARIC L. 2008 - Anciennes et nouvelles hypothèses d'interprétation du gravettien moyen en France : la question de la place des industries à burins du Raysse au sein de la mosaïque gravettienne, In : Le Gravettien : entités régionales d'une paléoculture europénne, 2 - L'Europe de l'Ouest, Table ronde des Eyzies 2004, Paléo 20, p. 257-276.

LADIER E. 2000 - Le Magdalénien ancien à lamelles à dos de l'abri Gandil à Bruniquel (Tarn-et-Garonne). Etude préliminaire de l'industrie lithique de la C20, In : G. Pion (Dir.), Le Paléolithique supérieur récent : nouvelles données sur le peuplement et l'environnement. Actes de la tableronde de Chambéry. 12-13 mars 1999. Mémoire, XXVIII, Paris, Société Préhistorique Française, p. 191-200, 5 fig.

LADIER E., WELTE A.-C. 1993 - Les objets de parure de la vallée de l'Aveyron. Fontalès, abris de Bruniquel (Plantade, Lafaye, Gandil). Paléo, 5, p. 281-317, 11 fig., 5 tabl., 2 diagr.

LADIER E., WELTE A.-C. 1994 - Bijoux de la préhistoire. La parure magdalénienne dans la vallée de l'Aveyron, Catalogue d'exposition des Muséum d'Histoire Naturelle de Montauban et de Toulouse 1, Mercuès, Publi-Offset, 191 p., 246 fig., IX tabl., 2 graph.

LANGLAIS M. 2007 - Dynamiques culturelles des sociétés magdaléniennes dans leurs cadres environnementaux. Enquête sur 7000 ans d'évolution de leurs industries lithiques entre Rhône et Ėbre. Thèse de doctorat en cotutelle avec les universités de Toulouse-Le Mirail et Barcelone, ex. multigraphié, 550 p.

LANGLAIS M., 2010 - Les sociétés magdaléniennes de l'isthme pyrénéen. Editions du CTHS, Paris, Documents Préhistoriques 26, 337 p.
LANGLAIS M., DUCASSE S., RENARD C., MORALA A. 2004 - L'exploitation des matériaux siliceux au Petit Cloup Barrat, caractérisation des ensembles lithiques magdaléniens, badegouliens et solutréens. In : J.-C. CASTEL, Petit Cloup Barrat, Cabrerets, Lot. Rapport de fouille programmée, été 2004, p. 68-80.

http://le-petit-cloup-barrat.fr ; rubrique « Rapports »

LANGLAIS M., LADIER E., CHALARD P., JARRY M., LACRAMPE-CUYAUBERE F., 2007 - Aux origines du Magdalénien "classique»: les industries de la séquence inférieure de l'Abri Gandil (Bruniquel, Tarn-et-Garonne). Paleo, 19, p. 341-366, 14 fig.

LANGLAIS M., PÉTILLON J.-M., BEAUNE de S.A., CHAUVIERE F.X., LETOURNEUX C., SZMIDT C., BEUKENS R. et DAVID F. 2010 a - Une occupation de la fin du dernier maximum glaciaire dans les Pyrénées : le Magdalénien inférieur de la grotte des Scilles (Lespugue, Haute-Garonne), Bulletin de la Société Préhistorique Française, 107 (1), p. 5-51.

LANGLAIS M., PÉTILLON J.-M., DUCASSE S. et LENOIR M. 2010b - Badegoulien versus Magdalénien. Entre choc culturel et lente transition dans l'Aquitaine paléolithique. In : V. Mistrot coord., De Néandertal à l'Homme Moderne. L'Aquitaine préhistorique, vingt ans de découvertes (19902010), p.116-129.

LANGLAIS M. et DUCASSE S., sous presse - Badegoulien versus Magdalénien. II - Le Magdalénien inferieur quercinois. In : Modalités d'occupations et exploitation des milieux au Paléolithique dans le Sud-Ouest de la France: l'exemple du Quercy, Actes du colloque 67 du XVème congres de I'UISPP, Lisbonne, Jarry M., Brugal J.-Ph. et Ferrier C. dir., Paleo.

LE BRUN-RICALENS F. et BROU L. 2003 - Burins carénésnucléus à lamelles : identification d'une chaine opératoire particulière a Thèmes (Yonne) et implications. Bulletin de la Société Préhistorique Française, 100(1), p. 67-83.

LEMOZI A. 1951 - Historique du Musée régional du château de Cabrerets. Activités diverses qui ont contribués à la formation et à l'expansion du Musée, Imprimerie Coueslant, Cahors, $15 \mathrm{p}$.

LENOIR M., MARMIER F., TRECOLLE G. 1991 - Données nouvelles sur les industries de Saint-Germain-la-Rivière (Gironde), In : 25 ans d'études technologiques en Préhistoire. Bilan et perspectives, actes des Xle Rencontres Internationales d'Archéologie et d'Histoire d'Antibes, 18-20 octobre 1990, Juan-les-Pins, APDCA, Juan-Les-Pins, ADPCA, p. 245-254, 4 fig.

LORBLANCHET M. 1972 - Aperçu sur le Magdalénien moyen et supérieur du Haut-Quercy, In : Congrès préhistorique de France, XIXè session, Auvergne, 1969, Paris, Société Préhistorique Française, p. 256-283, 15 fig.

LORBLANCHET M. avec la collaboration de F. DELPECH, P. RENAULT, C. ANDRIEUX., 1973 - La grotte de Sainte- 
Eulalie à Espagnac (Lot). Gallia Préhistoire, 16 (2) p. 233325.

MARKS A. et MISHOE M. 1997 - The Magdalenian of Portuguese Estremadura. In : El mon mediterrani després del Pleniglacial (18.000-12.000 BP), Actes du colloque de Banyoles 1995, Serie Monografica 17, Museu d'Arqueologia de Catalunya, p. 225-232.

MORALA A., 1993 - Technologie lithique du Magdalénien ancien de l'abri Casserole (Les Eyzies-de-Tayac, Dordogne). Paleo, 5, p. 193-208.

MORALA A. 2009 - Diagnostic industriel de la couche 10. In : Castel J.-C., Chauvière F.-X., Randon O., Camus, Ducasse S., Ferrand J., Langlais M., Morala A., Muth X., Renard C. et Turq A. - Petit Cloup Barrat, Cabrerets, Lot (46), rapport de fouille programmée deuxième triennale 2006-2008. Rapport d'évaluation à l'intention des services administratifs du Ministère de la Culture, p.199-205. http://le-petit-cloup-barrat.fr ; rubrique « Rapports »

NORMAND Ch. 1986 - Recherches de Préhistoire dans les Landes en 1985. Bulletin de la Société de Borda, 402, p. $132-140$.

PAJOT B. 1969 - Les civilisations du Paléolithique supérieur du bassin de l'Aveyron, Travaux de l'Institut d'Art Préhistorique 11, p. 584.

PELEGRIN J. et O'FARRELL M. 2005 - Les lamelles retouchées ou utilisées de Castanet. In : F. LebrunRicalens (ed.), avec la coll. de J.-G. Bordes et F. Bon, Productions lamellaires attribuées à l'Aurignacien : chaînes opératoires et perspectives technoculturelles. Luxembourg : Musée national d'Histoire, Collection Archéologiques, $n^{\circ} 1$, p. 103-121.

PERLES C. 1982 - Les «outils d'Orville»: des nucléus a lamelles. Studia Praehistorica Belgica, 2, p. 129-148.

PESCHAUX C. 2009 - La parure de la grotte du Taillis des Coteaux, In : La grotte du Taillis des Coteaux à Antigny (Vienne), J. Primault dir., rapport intermédiaire de la fouille programmée pluri-annuelle 2009-2011, p. 52-67. http://taillisdescoteaux.over-blog.net/article-5578895.html

PESCHAUX C. 2010 - Les éléments de parure de l'ensemble III découverts en 2010, In : La grotte du Taillis des Coteaux à Antigny (Vienne), J. Primault dir., rapport intermédiaire de la fouille programmée pluri-annuelle 2009-2011, p. 65-68. http://taillisdescoteaux.overblog.net/article-5578895.html

PÉTILLON J.-M. et AVERBOUH A., à paraitre - Le travail du bois de renne dans les couches badegouliennes. In : Solutréen et Badegoulien au Cuzoul de Vers. Des chasseurs de rennes en Quercy. Clottes J., Giraud J.- P. et Chalard P. dir., E.R.A.U.L.

PÉTILLON J.-M. et SACCHI D., à paraître - L'industrie osseuse. In : La station badegoulienne de Lassac à
Sallèles-Cabardès (Aude). Le Locus 1, éditions du GAEP, Carcassonne.

PÉTILLON J.-M., BIGNON O., BODU P., CATTELAIN P., DEBOUT G., LANGLAIS M., LAROULANDIE V., PLISSON H. et VALENTIN B. 2011 - Hard core and cutting edge: experimental manufacture and use of Magdalenian composite projectile tips, Journal of Archaeological Science, 38(6), p. 1-18.

http://www.sciencedirect.com/science/article/pii/S030544 0311000148

PÉTILLON J.-M., LANGLAIS M., A. De BAUNE S., CHAUVIERE F.-X., LETOURNEUX C., SZMIDT C., BEUKENS R. et DAVID F. 2008 - Le Magdalénien de la grotte des Scilles (Lespugue, Haute-Garonne). Premiers résultats de l'étude pluridisciplinaire de la collection Saint-Perier. Antiquités Nationales, 39, p. 57-71. http://halshs.archivesouvertes.fr/docs/00/40/37/07/PDF/Petillon_et_al_2008c.pdf

POPLIN F. 1983 - Incisives de renne sciées du Magdalénien d'Europe occidentale, In : La faune et l'homme préhistorique. Dix ans d'étude en hommage Jean Bouchud. Mémoire XVI, Paris, Société Préhistorique Française, p. 55-67, 9 fig.

PRIMAULT J., BROU L., GABILLEAU J. et LANGLAIS M. 2007 - La grotte du Taillis des Coteaux à Antigny (Vienne) : intérêts d'une séquence originale à la structuration des premiers temps du Magdalénien. Bulletin de la Société Préhistorique Française, 104(4), p. 743-758.

http://sd-1.archive-

host.com/membres/up/1470463121/2007N4743-

758Primault.pdf

PRIMAULT J. et coll. 2010 - La grotte du Taillis-desCoteaux à Antigny (Vienne), In : J. Buisson-Catil, J. Primault, (dir.), Préhistoire entre Vienne et Charente. Hommes et sociétés du Paléolithique, Mémoire XXXVIII, Chauvigny, Ministère de la culture et de la communication, Poitou-Charentes, p. 271-293, 26 fig.

PRIMAULT J., BROU L., BEAREZ P., GABILLEAU J., GRIGGO C., GUILLAUD E., DELFOUR G., HENRYGAMBIER D., HOUMARD C., JEANNET M., LAROULANDIE V., LILIOS D., LUCQUIN A., PASQUINI A., PESCHAUX C., PRIMAULT P., RAMBAUD D. et ROYER A. 2010 - La grotte du Taillis des Coteaux à Antigny (Vienne), rapport intermédiaire de la fouille programmée pluriannuelle 2009-2011, 110 p. http://taillisdescoteaux.overblog.net/article-5578895.html

RAUX P. 1994 - Un anneau ouvert en Périgord-Quercy. Bulletin de la Société Historique et Archéologique du Périgord, CXXI, p. 501-503.

RAUX P. et PIEL-DESRUISSEAUX J. 1997 - «La cave a endives» ou le Pech del Mas à Cabrerets (Lot). Etude de la collection André David. Bulletin de la Société Préhistorique Française, 94(1), p. 35-39. 
RENARD C. 2008 - Les premières expressions du Solutréen dans le Sud-Ouest français. Evolution technoéconomique des équipements lithiques au cours du dernier maximum glaciaire. Thèse de doctorat, Université de Nanterre-Paris X, 449 p.

RIGAUD A. 2004 - Fiche débitage du bois de renne au Magdalénien. L'exemple de La Garenne (Indre, France), In : D. Ramseyer (dir.), Matières et techniques. Fiches typologiques de l'ndustrie de l'os préhistorique, cahier XI, Paris, Société Préhistorique Française, p. 79-87.

RIGAUD A. 2007 - Retouchoirs sur éclats diaphysaires ou « affûtoirs » de Labastide (Hautes-Pyrénées). Du barbarisme scientifique à la rigueur artisanale au travers de l'expérimentation. Archéologie des Pyrénées occidentales et des Landes, 26, p. 193-200, 8 fig.

SACCHI D., BRÛLÉ J.-L. et DUCASSE S. dir., à paraître La station badegoulienne de Lassac à Sallèles-Cabardès (Aude). Le Locus 1, éditions du GAEP, Carcassonne.

SACKETT J. 1999 - The archaeology of Solvieux, an Upper Paleolithic open air site in France, Monumenta Archaeologica, 19, UCLA, Institute of Archaeology, Los Angeles, $328 \mathrm{p}$.

SÉRONIE-VIVIEN M.-R. 1995 - La grotte de Pégourié, Caniac-du-Causse, Lot : Périgordien, Badegoulien, Azilien, Age du Bronze, Préhistoire Quercinoise, supplément 2, $334 \mathrm{p}$.

SÉRONIE-VIVIEN M.-R. 2005 - L'industrie osseuse du Badegoulien de Pegourié (Caniac-du-Causse, Lot) et le décor pseudoexcisé. In : Industrie osseuse et parure du Solutréen au Magdalénien en Europe, Actes de la Table Ronde sur le Paléolithique supérieur récent, Angoulème, 28-30 mars 2003, Dujardin V. dir., p. 149-160.
SÉRONIE-VIVIEN M.-R., BENSCH C., CAPDEGELLE R., CHEVET B, 1981 - La grotte de la Bergerie des Quatre Chemins à Caniac du Causse (Lot). Gisement magdalénien et du Bronze ancien-Moyen, In : Congrès préhistorique de France, XXlè session, MontaubanCahors, 1979, Paris, Société Préhistorique Française, p. 266-286, 18 fig.

SÉRONIE-VIVIEN M., SÉRONIE-VIVIEN M.-R. et FOUCHER P. 2006 - L'économie du silex au Paléolithique supérieur dans le bassin d'Aquitaine. Le cas des silex a Lepidorbitoïdes des Pyrénées centrales. Caractérisation et implications méthodologiques. Paleo, 18, p. 193-216.

SIMONNET R. avec la collaboration de BON F., BORDES J.-G. et PRIMAULT J. 2007 - Le silex « Grains de Mil ». Localisation des gites. In : Frontières naturelles et frontières culturelles dans les Pyrénées préhistoriques, Table ronde de Tarascon-sur-Ariège, mars 2004, Ediciones de la Universidad de Cantabria, Santander, p. 101-102.

TARTAR E., TEYSSANDIER N., BON F. et LIOLIOS D. 2006 Equipement de chasse, équipement domestique : une distinction efficace ? Réflexion sur la notion d'investissement technique dans les industries aurignaciennes. In : Normes techniques et pratiques sociales : de la simplicité des outillages pré- et protohistoriques, Actes des XXVlèmes rencontres d'archéologie et d'histoire d'Antibes, 20-22 octobre 2005, Astruc L., Bon F., Lea V., Milcent P.-Y., Philibert S. dir., p. 107-117. 